\title{
Yamada-Watanabe Results for Stochastic Differential Equations with Jumps
}

\author{
Mátyás Barczy, ${ }^{1}$ Zenghu Li, ${ }^{2}$ and Gyula Pap ${ }^{3}$ \\ ${ }^{1}$ Faculty of Informatics, University of Debrecen, Pf. 12, Debrecen 4010, Hungary \\ ${ }^{2}$ School of Mathematical Sciences, Beijing Normal University, Beijing 100875, China \\ ${ }^{3}$ Bolyai Institute, University of Szeged, Aradi vértanúk tere 1, Szeged 6720, Hungary
}

Correspondence should be addressed to Mátyás Barczy; barczy.matyas@inf.unideb.hu

Received 27 May 2014; Revised 30 October 2014; Accepted 27 November 2014

Academic Editor: Agnès Sulem

Copyright (C) 2015 Mátyás Barczy et al. This is an open access article distributed under the Creative Commons Attribution License, which permits unrestricted use, distribution, and reproduction in any medium, provided the original work is properly cited.

Recently, Kurtz $(2007,2014)$ obtained a general version of the Yamada-Watanabe and Engelbert theorems relating existence and uniqueness of weak and strong solutions of stochastic equations covering also the case of stochastic differential equations with jumps. Following the original method of Yamada and Watanabe (1971), we give alternative proofs for the following two statements: pathwise uniqueness implies uniqueness in the sense of probability law, and weak existence together with pathwise uniqueness implies strong existence for stochastic differential equations with jumps.

\section{Introduction}

In order to prove existence and pathwise uniqueness of a strong solution for stochastic differential equations, it is an important issue to clarify the connections between weak and strong solutions. The first pioneering results are due to Yamada and Watanabe [1] for certain stochastic differential equations driven by Wiener processes.

We investigate stochastic differential equations with jumps. Let $U$ be a second-countable locally compact Hausdorff space equipped with its Borel $\sigma$-algebra $\mathscr{B}(U)$. Let $m$ be a $\sigma$-finite Radon measure on $(U, \mathscr{B}(U))$, meaning that the measure of compact sets is always finite. Let $U_{0}, U_{1} \in \mathscr{B}(U)$ be disjoint subsets. Let $d, r \in \mathbb{N}$. Let $b:[0, \infty) \times \mathbb{R}^{d} \rightarrow \mathbb{R}^{d}$, $\sigma:[0, \infty) \times \mathbb{R}^{d} \rightarrow \mathbb{R}^{d \times r}, f:[0, \infty) \times \mathbb{R}^{d} \times U \rightarrow \mathbb{R}^{d}$, and $g:[0, \infty) \times \mathbb{R}^{d} \times U \rightarrow \mathbb{R}^{d}$ be Borel measurable functions, where $[0, \infty) \times \mathbb{R}^{d} \times U$ is equipped with its Borel $\sigma$-algebra $\mathscr{B}\left([0, \infty) \times \mathbb{R}^{d} \times U\right)=\mathscr{B}([0, \infty)) \otimes \mathscr{B}\left(\mathbb{R}^{d}\right) \otimes \mathscr{B}(U)$ (see, e.g., Dudley [2, Proposition 4.1.7]). Consider a stochastic differential equation (SDE)

$$
\mathbf{X}_{t}=\mathbf{X}_{0}+\int_{0}^{t} b\left(s, \mathbf{X}_{s}\right) \mathrm{d} s+\int_{0}^{t} \sigma\left(s, \mathbf{X}_{s}\right) \mathrm{d} \mathbf{W}_{s}
$$

$$
\begin{aligned}
& +\int_{0}^{t} \int_{U_{0}} f\left(s, \mathbf{X}_{s-}, u\right) \widetilde{N}(\mathrm{~d} s, \mathrm{~d} u) \\
& +\int_{0}^{t} \int_{U_{1}} g\left(s, \mathbf{X}_{s-}, u\right) N(\mathrm{~d} s, \mathrm{~d} u), \quad t \in[0, \infty),
\end{aligned}
$$

where $\left(\mathbf{W}_{t}\right)_{t \geqslant 0}$ is an $r$-dimensional standard Brownian motion, $N(\mathrm{~d} s, \mathrm{~d} u)$ is a Poisson random measure on $(0, \infty) \times U$ with intensity measure $\mathrm{d} s m(\mathrm{~d} u), \widetilde{N}(\mathrm{~d} s, \mathrm{~d} u):=N(\mathrm{~d} s, \mathrm{~d} u)-$ $\mathrm{d} s m(\mathrm{~d} u)$, and $\left(\mathbf{X}_{t}\right)_{t \geqslant 0}$ is a suitable process with values in $\mathbb{R}^{d}$.

Yamada and Watanabe [1] proved that weak existence and pathwise uniqueness imply uniqueness in the sense of probability law and strong existence for the SDE (1) with $f=0$ and $g=0$. Engelbert [3] and Cherny [4] extended this result to a somewhat more general class of equations and gave a converse in which the roles of existence and uniqueness are reversed; that is, joint uniqueness in the sense of probability law (see, Engelbert [3, Definition 5]) and strong existence imply pathwise uniqueness. The original Yamada-Watanabe result arises naturally in the procedure of proving existence of solutions of a SDE; for a detailed discussion, see Kurtz [5, pages 1-2]. 
Jacod [6] generalized the above mentioned result of Yamada and Watanabe for a SDE driven by a semimartingale, where the coefficient may depend on the paths both of the solution and of the driving process. The Yamada-Watanabe result has been generalized by Ondreját [7] and Röckner et al. [8] for stochastic evolution equations in infinite dimensions and by Tappe [9] for semilinear stochastic partial differential equations with path-dependent coefficients.

Recently, there has been a renewed interest in generalizations of the results of Yamada and Watanabe [1]. Kurtz $[5,10]$ continued the direction of Engelbert [3] and Jacod [6]. He studied general stochastic models which relate stochastic inputs with stochastic outputs and obtained a general version of the Yamada-Watanabe and Engelbert theorems relating existence and uniqueness of weak and strong solutions of stochastic models with the message that the original results are not limited to SDEs driven by Wiener processes. In order to derive the original Yamada-Watanabe results from this general theory, proofs of pathwise uniqueness require appropriate adaptedness conditions, so two new notions, compatibility and partial compatibility between inputs and outputs, have been introduced. Due to Example 3.9 in Kurtz [10] and Page 7 in Kurtz [5], the results are valid for SDEs driven by a Wiener process and Poisson random measures.

Following the ideas of Yamada and Watanabe [1], we are going to give alternative proofs for the following two statements.

Theorem 1. Pathwise uniqueness for the SDE (1) implies uniqueness in the sense of probability law.

Theorem 2. Weak existence and pathwise uniqueness for the SDE (1) imply strong existence.

Note that Theorems 1 and 2 are generalizations of Proposition 1 and Corollary 1 in Yamada and Watanabe [1] (we do not intend to deal with generalization of their Corollary 3 ). The definition of weak and strong solutions of the SDE (1), pathwise uniqueness for the SDE (1) and uniqueness in the sense of probability law, and a detailed, precise formulation of Theorem 2 will be given in the paper. In the course of the proofs we developed a sequence of lemmas discussing several kinds of measurability; see Lemmas 12 and 14, and we also presented a key observation on the preservation of the joint distribution of the parts of the SDE (1); see Lemmas A.2 and A.4.

Our alternative proofs show the power of the original method of Yamada and Watanabe [1]; these proofs can be followed step by step and every technical detail is transparent in the paper. This raises a question whether Kurtz's result could be proved via the walked-out path by Yamada and Watanabe.

Note that Situ [11, Theorem 137] also considered the SDE (1) with $\mathbb{R}^{d} \backslash\{\mathbf{0}\}$ instead of $U$ and with $g=0$ and proved Theorems 1 and 2 under the resctrictive assumption

$$
\int_{\mathbb{R}^{d} \backslash\{0\}} \frac{\|u\|^{2}}{1+\|u\|^{2}} m(\mathrm{~d} u)<\infty
$$

This assumption was needed for introducing an auxiliary càdlàg process in Lemma 139 in Situ [11]. In fact, one can get rid of condition (2) by using the space of point measures on $\mathbb{R}_{+} \times U$ as the space of trajectories of Poisson point processes instead of the space of càdlàg functions; see the proofs of Theorems 1 and 2. We call the attention that in the literature the result of Situ [11, Theorem 137] has been usually referred to without checking condition (2); see, for example, $\mathrm{Li}$ and Mytnik [12, (3.1)], Dawson and Li [13, (2.9)], Döring and Barczy [14, (3.23)], and $\mathrm{Li}$ and $\mathrm{Pu}[15,(4.6)$ and (5.1)], but Theorem 2 covers these situations as well.

We remark that Zhao [16] already adapted the original method of Yamada and Watanabe for the SDE (1) driven only by a compensated Poisson random measure, that is, with $\sigma=0$ and $g=0$, but for processes with values in a separable Hilbert space instead of $\mathbb{R}^{d}$-valued processes. Comparing with the results of the present paper, note that we explicitly stated and proved in Theorem 1 that pathwise uniqueness for the SDE (1) implies uniqueness in the sense of probability law.

\section{Preliminaries}

Let $\mathbb{Z}_{+}, \mathbb{N}, \mathbb{R}, \mathbb{R}_{+}$, and $\mathbb{R}_{++}$denote the set of nonnegative integers, positive integers, real numbers, nonnegative real numbers, and positive real numbers, respectively. For $x, y \in$ $\mathbb{R}$, we will use the notation $x \wedge y:=\min \{x, y\}$. By $\|\mathbf{x}\|$ and $\|\mathbf{A}\|$, we denote the Euclidean norm of a vector $\mathbf{x} \in \mathbb{R}^{d}$ and the induced matrix norm of a matrix $\mathbf{A} \in \mathbb{R}^{d \times d}$, respectively. Throughout this paper, we make the conventions $\int_{a}^{b}:=\int_{(a, b]}$ and $\int_{a}^{\infty}:=\int_{(a, \infty)}$ for any $a, b \in \mathbb{R}$ with $a<b$. By $C\left(\mathbb{R}_{+}, \mathbb{R}^{\ell}\right)$ and $D\left(\mathbb{R}_{+}, \mathbb{R}^{\ell}\right)$, we denote the set of continuous and càdlàg $\mathbb{R}^{\ell}$-valued functions defined on $\mathbb{R}_{+}$, equipped with a metric inducing the local uniform topology (see, e.g., Jacod and Shiryaev [17, Section VI.1a]) and a metric inducing the socalled Skorokhod topology (see, e.g., Jacod and Shiryaev [17, Theorem VI.1.14]), respectively. Moreover, $\mathscr{C}\left(\mathbb{R}_{+}, \mathbb{R}^{\ell}\right)$ and $\mathscr{D}\left(\mathbb{R}_{+}, \mathbb{R}^{\ell}\right)$ denote the corresponding Borel $\sigma$-algebras on them.

Recall that $U$ is a second-countable locally compact Hausdorff space. Note that $U$ is homeomorphic to a separable complete metric space; see, for example, Kechris [18, Theorem 5.3]. For our later purposes, we recall the notion of the space of point measures on $\mathbb{R}_{+} \times U$, of the space of simple point measures on $\mathbb{R}_{+} \times U$, and of the vague convergence. We follow Resnick [19, Chapter 3] and Ikeda and Watanabe [20, Chapter I, Sections 8 and 9].

A point measure on $\mathbb{R}_{+} \times U$ is a measure $\pi$ of the following form: let $F \subset \mathbb{N}$ and let $\left\{\left(t_{i}, u_{i}\right): i \in F\right\}$ be a countable collection of (not necessarily distinct) points of $\mathbb{R}_{+} \times U$, and let

$$
\pi:=\sum_{i \in F} \delta_{\left(t_{i}, u_{i}\right)}
$$

assuming also that $\pi([0, t] \times B)<\infty$ for all $t \in \mathbb{R}_{+}$and compact subsets $B \in \mathscr{B}(U)$ (i.e., $\pi$ is a Radon measure meaning that the measure of compact sets is always finite, 
and consequently, it is locally finite), where $\delta_{\left(t_{i}, u_{i}\right)}$ denotes the Dirac measure concentrated on the point $\left(t_{i}, u_{i}\right)$. Thus

$$
\begin{array}{r}
\pi([0, t] \times B)=\#\left\{i \in F:\left(t_{i}, u_{i}\right) \in[0, t] \times B\right\}, \\
t \in \mathbb{R}_{+}, \quad B \in \mathscr{B}(U) .
\end{array}
$$

A point function (or point pattern) $p$ on $U$ is a mapping $p: D(p) \rightarrow U$, where the domain $D(p)$ is a countable subset of $\mathbb{R}_{++}$such that $\{s \in D(p): s \in(0, t], p(s) \in B\}$ is finite for all $t \in \mathbb{R}_{+}$and compact subsets $B \in \mathscr{B}(U)$. The counting measure $N_{p}$ on $\mathbb{R}_{++} \times U$ corresponding to $p$ is defined by

$$
\begin{array}{r}
N_{p}((0, t] \times B):=\#\{s \in D(p): s \in(0, t], p(s) \in B\}, \\
t \in \mathbb{R}_{++}, \quad B \in \mathscr{B}(U) .
\end{array}
$$

Note that there is a (natural) bijection between the set of point functions on $U$ and the set of point measures $\pi$ on $\mathbb{R}_{+} \times U$ with $\pi(\{t\} \times U) \leqslant 1, t \in \mathbb{R}_{++}$, and $\pi(\{0\} \times U)=0$. Namely, if $p: D(p) \rightarrow U$ is a point function, then the corresponding point measure is its counting measure $N_{p}=\sum_{t \in D(p)} \delta_{(t, p(t))}$. The set of all point measures on $\mathbb{R}_{+} \times U$ will be denoted by $M\left(\mathbb{R}_{+} \times U\right)$, and define a $\sigma$-algebra $\mathscr{M}\left(\mathbb{R}_{+} \times U\right)$ on it to be the smallest $\sigma$-algebra containing all sets of the form

$$
\begin{aligned}
& \left\{\pi \in M\left(\mathbb{R}_{+} \times U\right): \pi([0, t] \times B) \in A\right\} \\
& \text { for } t \in \mathbb{R}_{+}, \quad B \in \mathscr{B}(U), \quad A \in \mathscr{B}([0, \infty]) .
\end{aligned}
$$

Alternatively, $\mathscr{M}\left(\mathbb{R}_{+} \times U\right)$ is the smallest $\sigma$-algebra making all the mappings $M\left(\mathbb{R}_{+} \times U\right) \ni \pi \mapsto \pi([0, t] \times B) \in[0, \infty]$, $t \in \mathbb{R}_{+}, B \in \mathscr{B}(U)$, measurable.

Note that there is a (natural) bijection between the set of point processes (randomized point functions) $p$ defined on a probability space $(\Omega, \mathscr{F}, \mathbb{P})$ with values in the space of point functions on $U$ (in the sense of Ikeda and Watanabe [20, Chapter I, Definition 9.1]) and the set of $\mathscr{F} / \mathscr{M}\left(\mathbb{R}_{+} \times U\right)$ measurable mappings $p: \Omega \rightarrow M\left(\mathbb{R}_{+} \times U\right)$ with $p(\omega)(\{t\} \times$ $U) \leqslant 1$ for all $\omega \in \Omega$ and $t \in \mathbb{R}_{++}$and $p(\omega)(\{0\} \times U)=0$ for all $\omega \in \Omega$ (which are (special) point processes in the sense of Resnick [19, page 124]).

A point process $p$ on $U$ is called a Poisson point process if its counting measure $N_{p}$ is a Poisson random measure on $\mathbb{R}_{+} \times U$ (for the definition of Poisson random measure see, e.g., Ikeda and Watanabe [20, Chapter I, Definition 8.1]). A Poisson point process is stationary if and only if its intensity measure is of the form $\mathrm{d} s v(\mathrm{~d} u)$ for some measure $v$ on $(U, \mathscr{B}(U))$, which is called its charateristic measure. If $v$ is a Radon measure, then $N_{p}((0, t] \times B)$ is Poisson distributed with parameter $t \nu(B) \in \mathbb{R}_{+} ;$hence $\{s \in D(p): s \in(0, t], p(s) \in B\}$ is finite with probability one for all $t \in \mathbb{R}_{+}$and compact subsets $B \in \mathscr{B}(U)$. Consequently, a stationary Poisson point process with a Radon charateristic measure is a stationary Poisson point process in the sense of Ikeda and Watanabe [20, Chapter I, Definition 9.1].

Next we recall vague convergence. Let $C_{c}\left(\mathbb{R}_{+} \times U, \mathbb{R}_{+}\right)$be the space of $\mathbb{R}_{+}$-valued continuous functions defined on $\mathbb{R}_{+} \times$ $U$ with compact support. For $\pi, \pi_{n} \in M\left(\mathbb{R}_{+} \times U\right), n \in \mathbb{N}$, we say that $\pi_{n}$ converges vaguely to $\pi$ as $n \rightarrow \infty$ if

$$
\lim _{n \rightarrow \infty} \int_{\mathbb{R}_{+} \times U} f \mathrm{~d} \pi_{n}=\int_{\mathbb{R}_{+} \times U} f \mathrm{~d} \pi
$$

for all $f \in C_{c}\left(\mathbb{R}_{+} \times U, \mathbb{R}_{+}\right)$. For a topology on $M\left(\mathbb{R}_{+} \times U\right)$ giving this notion of convergence, see page 140 in Resnick [19]. Recall that $\mathscr{M}\left(\mathbb{R}_{+} \times U\right)$ coincides with the Borel $\sigma$-algebra generated by the open sets with respect to the vague topology on $M\left(\mathbb{R}_{+} \times U\right)$; see, for example, Resnick [19, Exercises 3.4.2(b) and 3.4.5].

In what follows we equip the spaces $C\left(\mathbb{R}_{+}, \mathbb{R}^{\ell}\right)$, $D\left(\mathbb{R}_{+}, \mathbb{R}^{\ell}\right), \ell \in \mathbb{N}$, and $M\left(\mathbb{R}_{+} \times U\right)$ with some $\sigma$-algebras that will be used later on. For each $\ell \in \mathbb{N}$, let us equip $C\left(\mathbb{R}_{+}, \mathbb{R}^{\ell}\right)$ and $D\left(\mathbb{R}_{+}, \mathbb{R}^{\ell}\right)$ with the $\sigma$-algebras

$$
\begin{aligned}
& \mathscr{C}_{t}\left(\mathbb{R}_{+}, \mathbb{R}^{\ell}\right):=\varphi_{t}^{-1}\left(\mathscr{C}\left(\mathbb{R}_{+}, \mathbb{R}^{\ell}\right)\right), \\
& \mathscr{D}_{t}\left(\mathbb{R}_{+}, \mathbb{R}^{\ell}\right):=\varphi_{t}^{-1}\left(\mathscr{D}\left(\mathbb{R}_{+}, \mathbb{R}^{\ell}\right)\right),
\end{aligned}
$$

for $t \in \mathbb{R}$, respectively, where $\varphi_{t}: D\left(\mathbb{R}_{+}, \mathbb{R}^{\ell}\right) \rightarrow D\left(\mathbb{R}_{+}, \mathbb{R}^{\ell}\right)$ is the mapping

$$
\left(\varphi_{t}(z)\right)(s):=z(t \wedge s), \quad z \in D\left(\mathbb{R}_{+}, \mathbb{R}^{\ell}\right), s \in \mathbb{R}_{+},
$$

which stops the function $z$ at $t$. It is easy to check that, for all $t \in \mathbb{R}_{+}, \mathscr{C}_{t}\left(\mathbb{R}_{+}, \mathbb{R}^{\ell}\right)$ coincides with the smallest $\sigma$-algebra containing all the finite-dimensional cylinder sets of the form

$$
\begin{gathered}
\left\{w \in C\left(\mathbb{R}_{+}, \mathbb{R}^{\ell}\right):\left(w\left(t_{1}\right), \ldots, w\left(t_{n}\right)\right) \in A\right\}, \\
n \in \mathbb{N}, \quad A \in \mathscr{B}\left(\mathbb{R}^{n \ell}\right), \quad t_{1}, \ldots, t_{n} \in[0, t],
\end{gathered}
$$

and then

$$
\mathscr{C}\left(\mathbb{R}_{+}, \mathbb{R}^{\ell}\right)=\sigma\left(\bigcup_{t \in \mathbb{R}_{+}} \mathscr{C}_{t}\left(\mathbb{R}_{+}, \mathbb{R}^{\ell}\right)\right)
$$

see, for example, Problem 2.4.2 in Karatzas and Shreve [21]. Similarly, for all $t \in \mathbb{R}_{+}, \mathscr{D}_{t}\left(\mathbb{R}_{+}, \mathbb{R}^{\ell}\right)$ coincides with the smallest $\sigma$-algebra containing all the finite-dimensional cylinder sets of the form

$$
\begin{aligned}
& \left\{y \in D\left(\mathbb{R}_{+}, \mathbb{R}^{\ell}\right):\left(y\left(t_{1}\right), \ldots, y\left(t_{n}\right)\right) \in A\right\}, \\
& n \in \mathbb{N}, \quad A \in \mathscr{B}\left(\mathbb{R}^{n \ell}\right), \quad t_{1}, \ldots, t_{n} \in[0, t],
\end{aligned}
$$

and then

$$
\mathscr{D}\left(\mathbb{R}_{+}, \mathbb{R}^{\ell}\right)=\sigma\left(\bigcup_{t \in \mathbb{R}_{+}} \mathscr{D}_{t}\left(\mathbb{R}_{+}, \mathbb{R}^{\ell}\right)\right) ;
$$

hence $\mathscr{D}_{t}\left(\mathbb{R}_{+}, \mathbb{R}^{\ell}\right)$ coincides with $\mathscr{D}_{t}^{0}\left(\mathbb{R}^{\ell}\right)$ in Definition VI.1.1 in Jacod and Shiryaev [17]. Finally, let us equip $M\left(\mathbb{R}_{+} \times U\right)$ with the $\sigma$-algebras $\mathscr{M}_{t}\left(\mathbb{R}_{+} \times U\right), t \in \mathbb{R}_{+}$, being the smallest $\sigma$-algebra containing all sets of the form

$$
\left\{\pi \in M\left(\mathbb{R}_{+} \times U\right): \pi([0, s] \times B) \in A\right\}
$$$$
\text { with } s \in[0, t], \quad B \in \mathscr{B}(U), \quad A \in \mathscr{B}([0, \infty]) \text {. }
$$

Note that

$$
\mathscr{M}\left(\mathbb{R}_{+} \times U\right)=\sigma\left(\bigcup_{t \in \mathbb{R}_{+}} \mathscr{M}_{t}\left(\mathbb{R}_{+} \times U\right)\right),
$$

since the union of the generator system of the $\sigma$-algebras $\mathscr{M}_{t}\left(\mathbb{R}_{+} \times U\right), t \in \mathbb{R}_{+}$, forms a generator system of $\mathscr{M}\left(\mathbb{R}_{+} \times U\right)$. 


\section{Notions of Weak and Strong Solutions}

If $(\Omega, \mathscr{F}, \mathbb{P})$ is a probability space, then, by $\mathbb{P}$-null sets from a sub- $\sigma$-algebra $\mathscr{H} \subset \mathscr{F}$, we mean the elements of the set

$$
\{A \subset \Omega: \exists B \in \mathscr{H} \text { such that } A \subset B, \mathbb{P}(B)=0\}
$$

Definition 3. Let $n$ be a probability measure on $\left(\mathbb{R}^{d}, \mathscr{B}\left(\mathbb{R}^{d}\right)\right)$. A weak solution of the $\operatorname{SDE}(1)$ with initial distribution $n$ is a tuple $\left(\Omega, \mathscr{F},\left(\mathscr{F}_{t}\right)_{t \in \mathbb{R}_{+}}, \mathbb{P}, \mathbf{W}, p, \mathbf{X}\right)$, where

(D1) $\left(\Omega, \mathscr{F},\left(\mathscr{F}_{t}\right)_{t \in \mathbb{R}_{+}}, \mathbb{P}\right)$ is a filtered probability space satisfying the usual hypotheses (i.e., $\left(\mathscr{F}_{t}\right)_{t \in \mathbb{R}_{+}}$is right continuous and $\mathscr{F}_{0}$ contains all the $\mathbb{P}$-null sets in $\left.\mathscr{F}\right)$;

(D2) $\left(\mathbf{W}_{t}\right)_{t \in \mathbb{R}_{+}}$is an $r$-dimensional standard $\left(\mathscr{F}_{t}\right)_{t \in \mathbb{R}_{+}}$ Brownian motion;

(D3) $p$ is a stationary $\left(\mathscr{F}_{t}\right)_{t \in \mathbb{R}_{+}}$-Poisson point process on $U$ with characteristic measure $m$;

(D4) $\left(\mathbf{X}_{t}\right)_{t \in \mathbb{R}_{+}}$is an $\mathbb{R}^{d}$-valued $\left(\mathscr{F}_{t}\right)_{t \in \mathbb{R}_{+}}$-adapted càdlàg process such that

(a) the distribution of $\mathbf{X}_{0}$ is $n$,

(b) $\mathbb{P}\left(\int_{0}^{t}\left(\left\|b\left(s, \mathbf{X}_{s}\right)\right\|+\left\|\sigma\left(s, \mathbf{X}_{s}\right)\right\|^{2}\right) \mathrm{d} s<\infty\right)=1, t \in$ $\mathbb{R}_{+}$,

(c) $\mathbb{P}\left(\int_{0}^{t} \int_{U_{0}}\left\|f\left(s, \mathbf{X}_{s}, u\right)\right\|^{2} \mathrm{~d} s m(\mathrm{~d} u)<\infty\right)=1, t \in$ $\mathbb{R}_{+}$,

(d) $\mathbb{P}\left(\int_{0}^{t} \int_{U_{1}}\left\|g\left(s, \mathbf{X}_{s^{-}}, u\right)\right\| N(\mathrm{~d} s, \mathrm{~d} u)<\infty\right)=1, t \in$ $\mathbb{R}_{+}$, where $N(\mathrm{~d} s, \mathrm{~d} u)$ is the counting measure of $p$ on $\mathbb{R}_{++} \times U$,

(e) equation (1) holds $\mathbb{P}$-a.s., where $\widetilde{N}(\mathrm{~d} s, \mathrm{~d} u)$ := $N(\mathrm{~d} s, \mathrm{~d} u)-\mathrm{d} s m(\mathrm{~d} u)$.

For the definitions of an $\left(\mathscr{F}_{t}\right)_{t \in \mathbb{R}_{+}}$-Brownian motion and an $\left(\mathscr{F}_{t}\right)_{t \in \mathbb{R}_{+}}$-Poisson point process, see, for example, Ikeda and Watanabe [20, Chapter I, Definition 7.2 and Chapter II, Definition 3.2].

In the next remark we point out that the integrals in the SDE (1) are well defined under the conditions of Definition 3 and have càdlàg modifications as functions of $t$.

Remark 4. If conditions (D1), (D2), and (D4)(b) are satisfied, then $\left(\int_{0}^{t} \sigma\left(s, \mathbf{X}_{s}\right) \mathrm{d} \mathbf{W}_{s}\right)_{t \in \mathbb{R}_{+}}$is well defined and has continuous sample paths almost surely; see, Ikeda and Watanabe [20, Chapter II, Definition 1.9]. Indeed, $\left(\sigma\left(t, \mathbf{X}_{t}\right)\right)_{t \in \mathbb{R}_{+}}$is $\left(\mathscr{F}_{t}\right)_{t \in \mathbb{R}_{+}}$-adapted (since $\mathbf{X}$ is $\left(\mathscr{F}_{t}\right)_{t \in \mathbb{R}_{+}}$-adapted and $\sigma$ is measurable), $\left(\sigma\left(t, \mathbf{X}_{t}\right)\right)_{t \in \mathbb{R}_{+}}$is measurable (since $\mathbf{X}$ is measurable, because it has right-continuous paths, see Karatzas and Shreve [21, Remark 1.1.14], and $\sigma$ is measurable), and $\mathbb{P}\left(\int_{0}^{t}\left\|\sigma\left(s, \mathbf{X}_{s}\right)\right\|^{2} \mathrm{~d} s<\infty\right)=1, t \in \mathbb{R}_{+}$.

Concerning conditions (D4)(c) and (d), note that the mappings $\mathbb{R}_{+} \times U_{0} \times \Omega \ni(s, u, \omega) \mapsto f\left(s, \mathbf{X}_{s-}(\omega), u\right) \in \mathbb{R}^{d}$ and $\mathbb{R}_{+} \times U_{1} \times \Omega \ni(s, u, \omega) \mapsto g\left(s, \mathbf{X}_{s-}(\omega), u\right) \in \mathbb{R}^{d}$ are $\left(\mathscr{F}_{t}\right)_{t \in \mathbb{R}_{+}}$-predictable; see Lemma A.1.

Hence condition (D4)(c) is satisfied if and only if the mapping $\mathbb{R}_{+} \times U_{0} \times \Omega \ni(s, u, \omega) \mapsto f\left(s, \mathbf{X}_{s-}(\omega), u\right) \in \mathbb{R}^{d}$ is in the (multidimensional version of the) class $\mathbf{F}_{p}^{2 \text {,loc }}$ defined on page 62 in Ikeda and Watanabe [20], that is, if it is $\left(\mathscr{F}_{t}\right)_{t \in \mathbb{R}_{+}}{ }^{-}$ predictable and there exists a sequence $\left(\tau_{n}\right)_{n \in \mathbb{N}}$ of $\left(\mathscr{F}_{t}\right)_{t \in \mathbb{R}_{+}}{ }^{-}$ stopping times such that $\tau_{n} \uparrow \infty$ almost surely as $n \rightarrow \infty$ and

$$
\begin{array}{r}
\mathbb{E}\left(\int_{0}^{t \wedge \tau_{n}} \int_{U_{0}}\left\|f\left(s, \mathbf{X}_{s}, u\right)\right\|^{2} \mathrm{~d} s m(\mathrm{~d} u)\right)<\infty, \\
t \in \mathbb{R}_{+}, \quad n \in \mathbb{N} .
\end{array}
$$

Indeed, if (D4)(c) holds then (17) is satisfied for

$$
\tau_{n}:=\inf \left\{t \in \mathbb{R}_{+}: \int_{0}^{t} \int_{U_{0}}\left\|f\left(s, \mathbf{X}_{s}, u\right)\right\|^{2} \mathrm{~d} s m(\mathrm{~d} u) \geqslant n\right\} \wedge n,
$$

for $n \in \mathbb{N}$, where $\tau_{n} \uparrow \infty$ almost surely as $n \rightarrow \infty$. On the other hand, (17) implies $\mathbb{P}\left(\int_{0}^{t \wedge \tau_{n}} \int_{U_{0}}\left\|f\left(s, \mathbf{X}_{s}, u\right)\right\|^{2} \mathrm{~d} s m(\mathrm{~d} u)<\right.$ $\infty)=1$ for all $t \in \mathbb{R}_{+}$and $n \in \mathbb{N}$, and hence (D4)(c), because $\tau_{n} \uparrow \infty$ almost surely as $n \rightarrow \infty$.

Moreover, if conditions (D1), (D3), and (D4)(c) are satisfied, then the process

$$
\left(\int_{0}^{t} \int_{U_{0}} f\left(s, \mathbf{X}_{s^{-}}, u\right) \widetilde{N}(\mathrm{~d} s, \mathrm{~d} u)\right)_{t \in \mathbb{R}_{+}}
$$

is well defined and has càdlàg sample paths almost surely. Indeed, for each $n \in \mathbb{N}$,

$$
\begin{gathered}
\left(\int_{0}^{t \wedge \tau_{n}} \int_{U_{0}} f\left(s, \mathbf{X}_{s-}, u\right) \widetilde{N}(\mathrm{~d} s, \mathrm{~d} u)\right)_{t \in \mathbb{R}_{+}} \\
\quad=\left(\int_{0}^{t} \int_{U_{0}} \mathbb{1}_{\left[0, \tau_{n}\right]}(s) f\left(s, \mathbf{X}_{s-}, u\right) \widetilde{N}(\mathrm{~d} s, \mathrm{~d} u)\right)_{t \in \mathbb{R}_{+}},
\end{gathered}
$$

see page 63 in Ikeda and Watanabe [20]. The integrand $\mathbb{R}_{+} \times U_{0} \times \Omega \ni(s, u, \omega) \mapsto \mathbb{1}_{\left[0, \tau_{n}\right]}(s) f\left(s, \mathbf{X}_{s-}(\omega), u\right) \in \mathbb{R}^{d}$ belongs to the (multidimensional version of the) class $\mathbf{F}_{p}^{2}$ defined on page 62 in Ikeda and Watanabe [20]; hence the process on the right hand side is a square integrable $\left(\mathscr{F}_{t}\right)_{t \in \mathbb{R}_{+}}{ }^{-}$ martingale; see page 63 in Ikeda and Watanabe [20]. By Theorem 1.3.13 in Karatzas and Shreve [21], this process has a càdlàg modification. Here we point out that, for using this theorem, we need completeness and right continuity of the filtration $\left(\mathscr{F}_{t}\right)_{t \in \mathbb{R}_{+}}$. Further, we also obtain

$$
\begin{aligned}
& \int_{0}^{t \wedge \tau_{n}} \int_{U_{0}} f\left(s, \mathbf{X}_{s-}, u\right) \widetilde{N}(\mathrm{~d} s, \mathrm{~d} u) \\
& \quad \stackrel{\text { a.s. }}{\longrightarrow} \int_{0}^{t} \int_{U_{0}} f\left(s, \mathbf{X}_{s-}, u\right) \widetilde{N}(\mathrm{~d} s, \mathrm{~d} u) \text { as } n \rightarrow \infty
\end{aligned}
$$

for all $t \in \mathbb{R}_{+}$, since $\tau_{n} \uparrow \infty$ almost surely as $n \rightarrow \infty$.

Recalling that the mapping $\mathbb{R}_{+} \times U_{1} \times \Omega \ni(s, u, \omega) \mapsto$ $g\left(s, \mathbf{X}_{s-}(\omega), u\right) \in \mathbb{R}^{d}$ is $\left(\mathscr{F}_{t}\right)_{t \in \mathbb{R}_{+}}$-predictable, condition (D4)(d) is satisfied if and only if the mapping $\mathbb{R}_{+} \times U_{1} \times \Omega \ni$ $(s, u, \omega) \mapsto g\left(s, \mathbf{X}_{s-}(\omega), u\right) \in \mathbb{R}^{d}$ is in the (multidimensional version of the) class $\mathbf{F}_{p}$ defined on page 61 in Ikeda and Watanabe [20]. 
Further, if conditions (D1), (D3), and (D4)(d) are satisfied, then, by definition, the process

$$
\begin{aligned}
& \left(\int_{0}^{t} \int_{U_{1}} g\left(s, \mathbf{X}_{s-}, u\right) N(\mathrm{~d} s, \mathrm{~d} u)\right)_{t \in \mathbb{R}_{+}} \\
& \quad=\left(\sum_{s \in(0, t] \cap D(p)} g\left(s, \mathbf{X}_{s-}, p(s)\right) \mathbb{1}_{U_{1}}(p(s))\right)_{t \in \mathbb{R}_{+}}
\end{aligned}
$$

is well defined and has càdlàg sample paths, where $D(p)$ is the domain of $p$ (being a countable subset of $\mathbb{R}_{++}$). Indeed, for each $\omega \in \Omega$, by definition, the mappings

$$
\begin{aligned}
& \mathbb{R}_{+} \ni t \\
& \longmapsto \sum_{s \in(0, t] \cap D(p)(\omega)} g\left(s, \mathbf{X}_{s-}(\omega), p(s)(\omega)\right) \mathbb{1}_{U_{1}}(p(s)(\omega)), \\
& \mathbb{R}_{+} \ni t \\
& \longmapsto \sum_{s \in(0, t) \cap D(p)(\omega)} g\left(s, \mathbf{X}_{s-}(\omega), p(s)(\omega)\right) \mathbb{1}_{U_{1}}(p(s)(\omega))
\end{aligned}
$$

are right and left continuous, respectively.

Remark 5. If $m\left(U_{1}\right)<\infty$, then condition (D4)(d) is satisfied automatically, since then $\mathbb{E}\left(N\left((0, t] \times U_{1}\right)=\operatorname{tm}\left(U_{1}\right)\right)<$ $\infty$ implies $\mathbb{P}\left(N\left((0, t] \times U_{1}\right)<\infty\right)=1$, and hence $\int_{0}^{t} \int_{U_{1}} \| g(s$, $\left.\mathbf{X}_{s-}, u\right)\left\|N(\mathrm{~d} s, \mathrm{~d} u)=\sum_{s \in(0, t] \cap D(p)}\right\| g\left(s, \mathbf{X}_{s-}, p(s)\right) \| \mathbb{1}_{U_{1}}(p(s))$ is a finite sum with probability one.

Remark 6. Note that if conditions (D1)-(D3) are satisfied, then $\mathbf{W}$ and $p$ are automatically independent according to Theorem 6.3 in Chapter II of Ikeda and Watanabe [20], since the intensity measure $\mathrm{d} s m(\mathrm{~d} u)$ of $p$ is deterministic.

Moreover, if $\left(\Omega, \mathscr{F},\left(\mathscr{F}_{t}\right)_{t \in \mathbb{R}}, \mathbb{P}, \mathbf{W}, p, \mathbf{X}\right)$ is a weak solution of the SDE (1), then $\mathscr{F}_{0}, \mathbf{W}$, and $p$ are mutually independent, and hence $\mathbf{X}_{0}, \mathbf{W}$, and $p$ are mutually independent as well. Indeed, the conditional joint charateristic function of $\mathbf{W}$ and the counting measure of $p$ with respect to $\mathscr{F}_{0}$ equals to the product of the (unconditional) charateristic functions of $\mathbf{W}$ and the counting measure of $p$; see (6.12) in Chapter II of Ikeda and Watanabe [20] applied with $X=\mathbf{W}$ and $s=0$, and then one can use Lemma 2.6.13 in Karatzas and Shreve [21]. Since $\mathbf{X}_{0}$ is measurable with respect to $\mathscr{F}_{0}$ due to (D4), we have the mutual independence of $\mathbf{X}_{0}, \mathbf{W}$, and $p$.

The thinnings $p_{0}$ and $p_{1}$ of $p$ onto $U_{0}$ and $U_{1}$ are again stationary $\left(\mathscr{F}_{t}\right)_{t \in \mathbb{R}_{+}}$-Poisson point processes on $U_{0}$ and $U_{1}$, respectively, and their characteristic measures are the restrictions $\left.m\right|_{U_{0}}$ and $\left.m\right|_{U_{1}}$ of $m$ onto $U_{0}$ and $U_{1}$, respectively (this can be checked calculating their conditional Laplace transforms; see Ikeda and Watanabe [20, page 44]).

Remark that for any weak solution of the SDE (1), $\mathbf{X}_{0}$, the Brownian motion $\mathbf{W}$ and the stationary Poisson point processes $p_{0}$ and $p_{1}$ are mutually independent according again to Theorem 6.3 in Chapter II of Ikeda and Watanabe [20]. Indeed, one can argue as before taking into account also that the intensity measures of $p_{0}$ and $p_{1}$ are deterministic, and condition (6.11) of this theorem is satisfied, because $p_{0}$ and $p_{1}$ live on disjoint subsets of $U$.

Definition 7. One says that pathwise uniqueness holds for the SDE (1) if whenever $\left(\Omega, \mathscr{F},\left(\mathscr{F}_{t}\right)_{t \in \mathbb{R}_{+}}, \mathbb{P}, \mathbf{W}, p, \mathbf{X}\right)$ and $\left(\Omega, \mathscr{F},\left(\mathscr{F}_{t}\right)_{t \in \mathbb{R}_{+}}, \mathbb{P}, \mathbf{W}, p, \widetilde{\mathbf{X}}\right)$ are weak solutions of the SDE (1) such that $\mathbb{P}\left(\mathbf{X}_{0}=\widetilde{\mathbf{X}}_{0}\right)=1$, then $\mathbb{P}\left(\mathbf{X}_{t}=\widetilde{\mathbf{X}}_{t}\right.$ for all $\left.t \in \mathbb{R}_{+}\right)=$ 1.

Remark 8. One may also consider the following more strict definition of pathwise uniqueness. Namely, one could say that pathwise uniqueness holds for the SDE (1) if when$\operatorname{ever}\left(\Omega, \mathscr{F},\left(\mathscr{F}_{t}\right)_{t \in \mathbb{R}_{+}}, \mathbb{P}, \mathbf{W}, p, \mathbf{X}\right)$ and $\left(\Omega, \mathscr{F},\left(\widetilde{\mathscr{F}}_{t}\right)_{t \in \mathbb{R}_{+}}, \mathbb{P}, \mathbf{W}\right.$, $p, \widetilde{\mathbf{X}})$ are weak solutions of the $\operatorname{SDE}(1)$ such that $\mathbb{P}\left(\mathbf{X}_{0}=\right.$ $\left.\widetilde{\mathbf{X}}_{0}\right)=1$, then $\mathbb{P}\left(\mathbf{X}_{t}=\widetilde{\mathbf{X}}_{t}\right.$ for all $\left.t \in \mathbb{R}_{+}\right)=1$. Note that in this definition we require that $\mathbf{W}$ is an $\left(\mathscr{F}_{t}\right)_{t \in \mathbb{R}_{+}}$-Brownian motion and an $\left(\widetilde{\mathscr{F}}_{t}\right)_{t \in \mathbb{R}_{+}}$-Brownian motion as well, and since it is not necessarily true that $\mathbf{W}$ is an $\left(\sigma\left(\mathscr{F}_{t} \cup \widetilde{\mathscr{F}}_{t}\right)\right)_{t \in \mathbb{R}_{+}}$-Brownian motion, it is not clear whether this more strict definition of pathwise uniqueness and the one given in Definition 7 are equivalent. According to Ikeda and Watanabe [20, Chapter IV, Remark 1.3], they are equivalent. We also point out that in our statements and proofs we use pathwise uniqueness in the sense of Definition 7, and we do not use the above mentioned equivalence of the two kinds of definitions.

Definition 9. One says that uniqueness in the sense of probability law holds for the $\operatorname{SDE}(1)$ if whenever $\left(\Omega, \mathscr{F},\left(\mathscr{F}_{t}\right)_{t \in \mathbb{R}_{+}}\right.$, $\mathbb{P}, \mathbf{W}, p, \mathbf{X})$ and $\left(\widetilde{\Omega}, \widetilde{\mathscr{F}},\left(\widetilde{\mathscr{F}}_{t}\right)_{t \in \mathbb{R}_{+}}, \widetilde{\mathbb{P}}, \widetilde{\mathbf{W}}, \widetilde{p}, \widetilde{\mathbf{X}}\right)$ are weak solutions of the SDE (1) with the same initial distribution, that is, $\mathbb{P}\left(\mathbf{X}_{0} \in B\right)=\widetilde{\mathbb{P}}\left(\widetilde{\mathbf{X}}_{0} \in B\right)$ for all $B \in \mathscr{B}\left(\mathbb{R}^{d}\right)$, then $\mathbb{P}(\mathbf{X} \in C)=$ $\widetilde{\mathbb{P}}(\widetilde{\mathbf{X}} \in C)$ for all $C \in \mathscr{D}\left(\mathbb{R}_{+}, \mathbb{R}^{d}\right)$.

Now we define strong solutions. Consider the following objects:

(E1) a probability space $(\Omega, \mathscr{F}, \mathbb{P})$;

(E2) an $r$-dimensional standard Brownian motion $\left(\mathbf{W}_{t}\right)_{t \in \mathbb{R}_{+}}$

(E3) a stationary Poisson point process $p$ on $U$ with characteristic measure $m$;

(E4) a random vector $\boldsymbol{\xi}$ with values in $\mathbb{R}^{d}$, independent of $\mathbf{W}$ and $p$.

Remark 10. Note that if conditions (E1)-(E4) are satisfied, then $\boldsymbol{\xi}, \mathbf{W}$, and $p$ are automatically mutually independent according to Remark 6.

Provided that the objects (E1)-(E4) are given, let $\left(\mathscr{F}_{t}^{\xi, \mathbf{W}, p}\right)_{t \in \mathbb{R}_{+}}$be the augmented filtration generated by $\boldsymbol{\xi}, \mathbf{W}$, and $p$; that is, for each $t \in \mathbb{R}_{+}, \mathscr{F}_{t}^{\xi, \mathrm{W}, p}$ is the $\sigma$-field generated by $\sigma\left(\xi ; \mathbf{W}_{s}, s \in[0, t] ; p(s), s \in(0, t] \cap D(p)\right)$ and by the $\mathbb{P}$ null sets from $\sigma\left(\xi ; \mathbf{W}_{s}, s \in \mathbb{R}_{+} ; p(s), s \in \mathbb{R}_{++} \cap D(p)\right)$ (which is similar to the definition in Karatzas and Shreve [21, page 285]). One can check that 
(i) $\left(\mathscr{F}_{t}^{\xi, \mathbf{W}, p}\right)_{t \in \mathbb{R}_{+}}$satisfies the usual hypotheses;

(ii) $\left(\mathbf{W}_{t}\right)_{t \in \mathbb{R}_{+}}$is a standard $\left(\mathscr{F}_{t}^{\xi, \mathbf{W}, p}\right)_{t \in \mathbb{R}_{+}}$-Brownian motion;

(iii) $p$ is a stationary $\left(\mathscr{F}_{t}^{\xi, \mathbf{W}, p}\right)_{t \in \mathbb{R}_{+}}$-Poisson point process on $U$ with characteristic measure $m$.

Indeed, by Remark $10, \mathbf{W}$ is a standard $\left(\sigma\left(\xi ; \mathbf{W}_{s}, s \in[0, t]\right.\right.$; $p(s), s \in(0, t] \cap D(p)))_{t \in \mathbb{R}_{+}}$-Brownian motion, and $p$ is a stationary $\left(\sigma\left(\xi ; \mathbf{W}_{s}, s \in[0, t] ; p(s), s \in(0, t] \cap D(p)\right)\right)_{t \in \mathbb{R}_{+}{ }^{-}}$ Poisson point process on $U$ with characteristic measure $m$. Hence, by Theorems 6.4 and 6.5 in Chapter II in Ikeda and Watanabe [20], (W, $p)$ has the strong Markov property with respect to the filtration $\left(\sigma\left(\xi ; \mathbf{W}_{s}, s \in[0, t] ; p(s), s \in\right.\right.$ $(0, t] \cap D(p)))_{t \in \mathbb{R}_{+}}$. Then Proposition 2.7.7 in Karatzas and Shreve [21] yields that the augmented filtration $\left(\mathscr{F}_{t}^{\xi, W, p}\right)_{t \in \mathbb{R}_{+}}$ satisfies the usual hypotheses. Moreover, the augmentation of $\sigma$-fields does not disturb the definition of a standard Wiener process and a stationary Poisson point process; hence $\left(\mathbf{W}_{t}\right)_{t \in \mathbb{R}_{+}}$is a standard $\left(\mathscr{F}_{t}^{\xi, \mathbf{W}, p}\right)_{t \in \mathbb{R}_{+}}$-Brownian motion, and $p$ is a stationary $\left(\mathscr{F}_{t}^{\xi, \mathrm{W}, p}\right)_{t \in \mathbb{R}_{+}}$-Poisson point process on $U$ with characteristic measure $m$. For the standard Wiener process, see, for example, Karatzas and Shreve [21, Theorem 2.7.9]. The main point is to show that $\mathbf{W}_{t}-\mathbf{W}_{s}$ is independent of $\mathscr{F}_{s}^{\xi, \mathbf{W}, p}$ for all $s, t \in \mathbb{R}_{+}$with $s<t$, and $p(t)-p(s)$ is independent of $\mathscr{F}_{s}^{\xi, \mathbf{W}, p}$ for all $s, t \in D(p)$ with $s<t$, detailed as follows (in order to shed some light what is going on behind). Let $s, t \in \mathbb{R}_{+}$with $s<t$, and $F \in \mathscr{F}_{s}^{\xi, W}, p$. Then, by Problem 2.7.3 in Karatzas and Shreve [21], there exists $\widetilde{F} \in \sigma\left(\boldsymbol{\xi} ; \mathbf{W}_{u}, u \in\right.$ $[0, s] ; p(u), u \in(0, s] \cap D(p))$ such that $F \Delta \widetilde{F}$ is a $\mathbb{P}$-null set from $\sigma\left(\xi ; \mathbf{W}_{u}, u \in \mathbb{R}_{+} ; p(u), u \in \mathbb{R}_{++} \cap D(p)\right)$, where $F \Delta \widetilde{F}$ denotes the symmetric difference of $F$ and $\widetilde{F}$. Using that

$$
\begin{aligned}
\mathbb{P}(A)= & \mathbb{P}(B)+\mathbb{P}(A \cap(\Omega \backslash B)) \\
& -\mathbb{P}((\Omega \backslash A) \cap B), \quad A, B \in \mathscr{F},
\end{aligned}
$$

we get for all $K \in \mathscr{B}\left(\mathbb{R}^{r}\right)$,

$$
\begin{aligned}
\mathbb{P} & \left(\left\{\mathbf{W}_{t}-\mathbf{W}_{s} \in K\right\} \cap F\right) \\
= & \mathbb{P}\left(\left\{\mathbf{W}_{t}-\mathbf{W}_{s} \in K\right\} \cap \widetilde{F}\right) \\
& +\mathbb{P}\left(\left\{\mathbf{W}_{t}-\mathbf{W}_{s} \in K\right\} \cap F \cap\left(\left\{\mathbf{W}_{t}-\mathbf{W}_{s} \notin K\right\} \cup(\Omega \backslash \widetilde{F})\right)\right) \\
& -\mathbb{P}\left(\left(\left\{\mathbf{W}_{t}-\mathbf{W}_{s} \notin K\right\} \cup(\Omega \backslash F)\right) \cap\left\{\mathbf{W}_{t}-\mathbf{W}_{s} \in K\right\} \cap \widetilde{F}\right) \\
= & \mathbb{P}\left(\left\{\mathbf{W}_{t}-\mathbf{W}_{s} \in K\right\} \cap \widetilde{F}\right) \\
& +\mathbb{P}\left(\left\{\mathbf{W}_{t}-\mathbf{W}_{s} \in K\right\} \cap F \cap(\Omega \backslash \widetilde{F})\right) \\
& -\mathbb{P}\left(\left\{\mathbf{W}_{t}-\mathbf{W}_{s} \in K\right\} \cap(\Omega \backslash F) \cap \widetilde{F}\right) \\
= & \mathbb{P}\left(\left\{\mathbf{W}_{t}-\mathbf{W}_{s} \in K\right\} \cap \widetilde{F}\right)=\mathbb{P}\left(\mathbf{W}_{t}-\mathbf{W}_{s} \in K\right) \mathbb{P}(\widetilde{F}) \\
= & \mathbb{P}\left(\mathbf{W}_{t}-\mathbf{W}_{s} \in K\right) \mathbb{P}(F),
\end{aligned}
$$

where the last but one step follows from the independence of $\mathbf{W}_{t}-\mathbf{W}_{s}$ and $\widetilde{F}$. A similar argument shows the independence of $p(t)-p(s)$ and $F$.

Definition 11. Suppose that the objects (E1)-(E4) are given. A strong solution of the $\operatorname{SDE}(1)$ on $(\Omega, \mathscr{F}, \mathbb{P})$ and with respect to the standard Brownian motion $\mathbf{W}$, the stationary Poisson point process $p$ and initial value $\xi$, is an $\mathbb{R}^{d}$-valued $\left(\mathscr{F}_{t}^{\xi, \mathbf{W}, p}\right)_{t \in \mathbb{R}_{+}}$-adapted càdlàg process $\left(\mathbf{X}_{t}\right)_{t \in \mathbb{R}_{+}}$with $\mathbb{P}\left(\mathbf{X}_{0}=\right.$ $\xi)=1$ satisfying $(\mathrm{D} 4)(\mathrm{b})-(\mathrm{d})$.

Clearly, if $\left(\mathbf{X}_{t}\right)_{t \in \mathbb{R}_{+}}$is a strong solution, then $(\Omega, \mathscr{F}$, $\left.\left(\mathscr{F}_{t}^{\xi, \mathbf{W}, p}\right)_{t \in \mathbb{R}_{+}}, \mathbb{P}, \mathbf{W}, p, \mathbf{X}\right)$ is a weak solution with initial distribution being the distribution of $\xi$.

\section{Proof of Theorem 1}

Our presentation as follows is a generalization of the one given in Section 5.3.D in Karatzas and Shreve [21].

Let us consider a weak solution $\left(\Omega, \mathscr{F},\left(\mathscr{F}_{t}\right)_{t \in \mathbb{R}_{+}}, \mathbb{P}, \mathbf{W}\right.$, $p, \mathbf{X})$ of the $\operatorname{SDE}(1)$ with initial distribution $n$ on $\left(\mathbb{R}^{d}\right.$, $\left.\mathscr{B}\left(\mathbb{R}^{d}\right)\right)$. Then $\mathbb{P}\left(\mathbf{X}_{0} \in B\right)=n(B), B \in \mathscr{B}\left(\mathbb{R}^{d}\right)$. We put $\mathbf{Y}_{t}:=$ $\mathbf{X}_{t}-\mathbf{X}_{0}$ for $t \in \mathbb{R}_{+}$, and we regard the solution $\mathbf{X}$ as consisting of four parts: $\mathbf{X}_{0}, \mathbf{W}, p$, and $\mathbf{Y}$. Let us consider the product space

$$
\Theta:=\mathbb{R}^{d} \times C\left(\mathbb{R}_{+}, \mathbb{R}^{r}\right) \times M\left(\mathbb{R}_{+} \times U\right) \times D\left(\mathbb{R}_{+}, \mathbb{R}^{d}\right)
$$

equipped with the Borel $\sigma$-algebra

$$
\begin{aligned}
\mathscr{B}(\Theta)= & \mathscr{B}\left(\mathbb{R}^{d}\right) \otimes \mathscr{C}\left(\mathbb{R}_{+}, \mathbb{R}^{r}\right) \otimes \mathscr{M}\left(\mathbb{R}_{+} \times U\right) \\
& \otimes \mathscr{D}\left(\mathbb{R}_{+}, \mathbb{R}^{d}\right) ;
\end{aligned}
$$

see, for example, Dudley [2, Proposition 4.1.7]. The quadruplet $\left(\mathbf{X}_{0}, \mathbf{W}, p, \mathbf{Y}\right)$ induce the probability measure $P$ on $(\Theta$, $\mathscr{B}(\Theta))$ according to the prescription

$$
P(A):=\mathbb{P}\left[\left(\mathbf{X}_{0}, \mathbf{W}, p, \mathbf{Y}\right) \in A\right], \quad A \in \mathscr{B}(\Theta) .
$$

We denote by $\theta=(\mathbf{x}, w, \pi, y)$ a generic element of $\Theta$. The marginal of $P$ on the $\mathbf{x}$-coordinate of $\theta$ is the probability measure $n$ on $\left(\mathbb{R}^{d}, \mathscr{B}\left(\mathbb{R}^{d}\right)\right)$, the marginal on the $w$-coordinate is an $r$-dimensional Wiener measure $P_{\mathbf{W}, r}$ on $\left(C\left(\mathbb{R}_{+}, \mathbb{R}^{r}\right)\right.$, $\mathscr{C}\left(\mathbb{R}_{+}, \mathbb{R}^{r}\right)$ ), the marginal on the $\pi$-coordinate is the distribution $P_{U, m}$ on $\left(M\left(\mathbb{R}_{+} \times U\right), \mathscr{M}\left(\mathbb{R}_{+} \times U\right)\right)$ of a stationary Poisson point process $p$ on $U$ with characteristic measure $m$. Moreover, the distribution of the triplet $(\mathbf{x}, w, \pi)$ under $P$ is the product measure $n \times P_{\mathrm{W}, r} \times P_{U, m}$ because $\mathbf{X}_{0}$ is $\mathscr{F}_{0^{-}}$measurable and $\mathbf{W}, p$, and $\mathscr{F}_{0}$ are independent; see Remark 6 . Furthermore, $\mathbb{P}\left(\mathbf{Y}_{0}=\mathbf{0}\right)=1$.

The product space $\Theta$ defined in (26) is a complete, separable metric space, since $\mathbb{R}^{d}$ is a complete, separable metric space with the usual Euclidean metric, $C\left(\mathbb{R}_{+}, \mathbb{R}^{r}\right)$ is a complete, separable metric space with a metric inducing the local uniform topology (see, e.g., Jacod and Shiryaev 
[17, Section VI.1a]), $D\left(\mathbb{R}_{+}, \mathbb{R}^{d}\right)$ is a complete, separable metric space with a metric inducing the so-called Skorokhod topology (see, e.g., Jacod and Shiryaev [17, Theorem VI.1.14]), and the vague topology on the space $M\left(\mathbb{R}_{+} \times U\right)$ of all point measures on $\mathbb{R}_{+} \times U$ is metrizable as a complete, separable metric space (see, e.g., Resnick [19, Proposition 3.17, page 147]). Hence there exists a regular conditional probability for $\mathscr{B}(\Theta)$ given $(\mathbf{x}, w, \pi)$, by an application of Karatzas and Shreve [21, Chapter 5, Theorem 3.19] with the random variable $\Theta \ni(\mathbf{x}, w, \pi, y) \mapsto(\mathbf{x}, w, \pi)$. We will be interested in conditional probabilities of sets in $\mathscr{B}(\Theta)$ only of the form $\mathbb{R}^{d} \times C\left(\mathbb{R}_{+}, \mathbb{R}^{r}\right) \times M\left(\mathbb{R}_{+} \times U\right) \times F$, where $F \in$ $\mathscr{D}\left(\mathbb{R}_{+}, \mathbb{R}^{d}\right)$. Consequently, with a slight abuse of notation, there exists a function

$$
Q: \mathbb{R}^{d} \times C\left(\mathbb{R}_{+}, \mathbb{R}^{r}\right) \times M\left(\mathbb{R}_{+} \times U\right) \times \mathscr{D}\left(\mathbb{R}_{+}, \mathbb{R}^{d}\right) \longrightarrow[0,1]
$$

enjoying the following properties:

(R1) for each $\mathbf{x} \in \mathbb{R}^{d}, w \in C\left(\mathbb{R}_{+}, \mathbb{R}^{r}\right)$ and $\pi \in M\left(\mathbb{R}_{+} \times U\right)$, the set function $\mathscr{D}\left(\mathbb{R}_{+}, \mathbb{R}^{d}\right) \ni F \mapsto Q(\mathbf{x}, w, \pi, F)$ is a probability measure on $\left(D\left(\mathbb{R}_{+}, \mathbb{R}^{d}\right), \mathscr{D}\left(\mathbb{R}_{+}, \mathbb{R}^{d}\right)\right)$;

(R2) for each $F \in \mathscr{D}\left(\mathbb{R}_{+}, \mathbb{R}^{d}\right)$, the mapping $\mathbb{R}^{d} \times C\left(\mathbb{R}_{+}\right.$, $\left.\mathbb{R}^{r}\right) \times M\left(\mathbb{R}_{+} \times U\right) \ni(\mathbf{x}, w, \pi) \mapsto Q(\mathbf{x}, w, \pi, F)$ is $\mathscr{B}\left(\mathbb{R}^{d}\right) \otimes \mathscr{C}\left(\mathbb{R}_{+}, \mathbb{R}^{r}\right) \otimes \mathscr{M}\left(\mathbb{R}_{+} \times U\right) / \mathscr{B}([0,1])$-measurable;

(R3) for each $G \in \mathscr{B}\left(\mathbb{R}^{d}\right) \otimes \mathscr{C}\left(\mathbb{R}_{+}, \mathbb{R}^{r}\right) \otimes \mathscr{M}\left(\mathbb{R}_{+} \times U\right)$ and $F \in \mathscr{D}\left(\mathbb{R}_{+}, \mathbb{R}^{d}\right)$, we have

$$
P(G \times F)=\int_{G} Q(\mathbf{x}, w, \pi, F) n(\mathrm{~d} \mathbf{x}) P_{\mathrm{W}, r}(\mathrm{~d} w) P_{U, m}(\mathrm{~d} \pi) .
$$

We can call $Q(\mathbf{x}, w, \pi, \cdot)$ as the regular conditional probability for $\mathscr{D}\left(\mathbb{R}_{+}, \mathbb{R}^{d}\right)$ given $(\mathbf{x}, w, \pi)$.

Let us now consider two weak solutions $\left(\Omega^{(i)}, \mathscr{F}^{(i)}\right.$, $\left.\left(\mathscr{F}_{t}^{(i)}\right)_{t \in \mathbb{R}_{+}}, \mathbb{P}^{(i)}, \mathbf{W}^{(i)}, p^{(i)}, \mathbf{X}^{(i)}\right), i \in\{1,2\}$ of the SDE (1) with the same initial distribution $n$ on $\left(\mathbb{R}^{d}, \mathscr{B}\left(\mathbb{R}^{d}\right)\right)$; thus

$$
\mathbb{P}^{(1)}\left[\mathbf{X}_{0}^{(1)} \in B\right]=\mathbb{P}^{(2)}\left[\mathbf{X}_{0}^{(2)} \in B\right]=n(B), \quad B \in \mathscr{B}\left(\mathbb{R}^{d}\right) .
$$

According to (28), let

$$
\begin{array}{r}
P_{i}(A):=\mathbb{P}^{(i)}\left[\left(\mathbf{X}_{0}^{(i)}, \mathbf{W}^{(i)}, p^{(i)}, \mathbf{Y}^{(i)}\right) \in A\right], \\
A \in \mathscr{B}(\Theta), \quad i \in\{1,2\},
\end{array}
$$

and, as explained before, there exist functions

$$
\begin{aligned}
Q_{i}: \mathbb{R}^{d} & \times C\left(\mathbb{R}_{+}, \mathbb{R}^{r}\right) \times M\left(\mathbb{R}_{+} \times U\right) \\
& \times \mathscr{D}\left(\mathbb{R}_{+}, \mathbb{R}^{d}\right) \longrightarrow[0,1], \quad i \in\{1,2\},
\end{aligned}
$$

enjoying the properties (R1)-(R3).

First, we bring the two triplets $\left(\mathbf{X}^{(i)}, \mathbf{W}^{(i)}, p^{(i)}\right), i \in\{1,2\}$, together on the same canonical space, while preserving the joint distribution of the coordinates within each triplet. Let $\Omega:=\Theta \times D\left(\mathbb{R}_{+}, \mathbb{R}^{d}\right)$ equipped with the $\sigma$-algebra $\mathscr{F}$, which is the completion of the product $\sigma$-algebra $\mathscr{B}(\Theta) \otimes \mathscr{D}\left(\mathbb{R}_{+}, \mathbb{R}^{d}\right)$ by the collection $\mathscr{N}$ of null sets under the probability measure

$$
\begin{aligned}
& \mathbb{P}_{1,2}(A):=\int_{\mathbb{R}^{d} \times C\left(\mathbb{R}_{+}, \mathbb{R}^{r}\right) \times M\left(\mathbb{R}_{+} \times U\right)}\left(\int_{D\left(\mathbb{R}_{+}, \mathbb{R}^{d}\right) \times D\left(\mathbb{R}_{+}, \mathbb{R}^{d}\right)} \mathbb{1}_{A}\left(\mathbf{x}, w, \pi, y^{(1)}, y^{(2)}\right) Q_{1}\left(\mathbf{x}, w, \pi, \mathrm{d} y^{(1)}\right) Q_{2}\left(\mathbf{x}, w, \pi, \mathrm{d} y^{(2)}\right)\right) \\
& n(\mathrm{~d} \mathbf{x}) P_{\mathbf{W}, r}(\mathrm{~d} w) P_{U, m}(\mathrm{~d} \pi)
\end{aligned}
$$

for $A \in \mathscr{B}(\Theta) \otimes \mathscr{D}\left(\mathbb{R}_{+}, \mathbb{R}^{d}\right)$, where we have denoted by $\left(\mathbf{x}, w, \pi, y^{(1)}, y^{(2)}\right)$ a generic element of $\Omega$, and then we extend $\mathbb{P}_{1,2}$ to $\mathscr{F}$. Particularly, for all $G \in \mathscr{B}\left(\mathbb{R}^{d}\right) \otimes \mathscr{C}\left(\mathbb{R}_{+}, \mathbb{R}^{r}\right) \otimes$ $\mathscr{M}\left(\mathbb{R}_{+} \times U\right)$ and $F_{1}, F_{2} \in \mathscr{D}\left(\mathbb{R}_{+}, \mathbb{R}^{d}\right)$,

$$
\begin{gathered}
\mathbb{P}_{1,2}\left(G \times F_{1} \times F_{2}\right) \\
=\int_{G} Q_{1}\left(\mathbf{x}, w, \pi, F_{1}\right) Q_{2}\left(\mathbf{x}, w, \pi, F_{2}\right) \\
n(\mathrm{~d} \mathbf{x}) P_{\mathbf{W}, r}(\mathrm{~d} w) P_{U, m}(\mathrm{~d} \pi) .
\end{gathered}
$$

In order to endow $\left(\Omega, \mathscr{F}, \mathbb{P}_{1,2}\right)$ with a filtration that satisfies the usual conditions, for each $t \in \mathbb{R}_{+}$, we take $\mathscr{G}_{t}:=\sigma\left(f_{s, B}\right.$ : $s \in[0, t], B \in \mathscr{B}(U))$, where the mapping $f_{s, B}: \Omega \rightarrow \mathbb{R}^{d} \times$ $\mathbb{R}^{r} \times[0, \infty] \times \mathbb{R}^{d} \times \mathbb{R}^{d}$ is defined by

$$
\begin{array}{r}
f_{s, B}\left(\mathbf{x}, w, \pi, y^{(1)}, y^{(2)}\right):=\left(\mathbf{x}, w_{s}, \pi([0, s] \times B), y_{s}^{(1)}, y_{s}^{(2)}\right), \\
\left(\mathbf{x}, w, \pi, y^{(1)}, y^{(2)}\right) \in \Omega,
\end{array}
$$

and put

$$
\widetilde{\mathscr{G}}_{t}:=\sigma\left(\mathscr{G}_{t} \cup \mathcal{N}\right), \quad \mathscr{F}_{t}:=\widetilde{\mathscr{G}}_{t+}:=\bigcap_{\varepsilon>0} \widetilde{\mathscr{G}}_{t+\mathcal{E}}, \quad t \in \mathbb{R}_{+} .
$$

We note that, for each $t \in \mathbb{R}_{+}$,

$$
\begin{aligned}
\mathscr{G}_{t}=\widehat{\mathscr{G}}_{t}= & \mathscr{B}\left(\mathbb{R}^{d}\right) \otimes \mathscr{C}_{t}\left(\mathbb{R}_{+}, \mathbb{R}^{r}\right) \otimes \mathscr{M}_{t}\left(\mathbb{R}_{+} \times U\right) \\
& \otimes \mathscr{D}_{t}\left(\mathbb{R}_{+}, \mathbb{R}^{d}\right) \otimes \mathscr{D}_{t}\left(\mathbb{R}_{+}, \mathbb{R}^{d}\right),
\end{aligned}
$$

where $\widehat{\mathscr{G}}_{t}:=\sigma\left(\widehat{f}_{s, B}: s \in[0, t], B \in \mathscr{B}(U)\right)$, and the mapping $\widehat{f}_{s, B}: \Omega \rightarrow \Omega$ is defined by

$$
\begin{aligned}
& \widehat{f}_{s, B}\left(\mathbf{x}, w, \pi, y^{(1)}, y^{(2)}\right) \\
& \quad:=\left(\mathbf{x},\left(w_{t \wedge s}\right)_{t \in \mathbb{R}_{+}},\left.\pi\right|_{[0, s] \times B},\left(y_{t \wedge s}^{(1)}\right)_{t \in \mathbb{R}_{+}},\left(y_{t \wedge s}^{(2)}\right)_{t \in \mathbb{R}_{+}}\right)
\end{aligned}
$$


for $\left(\mathbf{x}, w, \pi, y^{(1)}, y^{(2)}\right) \in \Omega$. Indeed, for all $t \in \mathbb{R}_{+}$, by definition, the $\sigma$-algebra $\mathscr{G}_{t}$ coincides with the $\sigma$-algebra generated by the sets

$$
\begin{aligned}
E_{1} & \times\left\{w \in C\left(\mathbb{R}_{+}, \mathbb{R}^{r}\right): w(s) \in E_{2}\right\} \\
& \times\left\{\pi \in M\left(\mathbb{R}_{+} \times U\right): \pi([0, s] \times B) \in E_{3}\right\} \\
& \times\left\{y^{(1)} \in D\left(\mathbb{R}_{+}, \mathbb{R}^{d}\right): y^{(1)}(s) \in E_{4}\right\} \\
& \times\left\{y^{(2)} \in D\left(\mathbb{R}_{+}, \mathbb{R}^{d}\right): y^{(2)}(s) \in E_{5}\right\}
\end{aligned}
$$

for $s \in[0, t], B \in \mathscr{B}(U), E_{1} \in \mathscr{B}\left(\mathbb{R}^{d}\right), E_{2} \in \mathscr{B}\left(\mathbb{R}^{r}\right), E_{3} \in$ $\mathscr{B}([0, \infty])$, and $E_{4}, E_{5} \in \mathscr{B}\left(\mathbb{R}^{d}\right)$. Moreover, as in Problem 2.4.2 in Karatzas and Shreve [21], the $\sigma$-algebra $\widehat{\mathscr{G}}_{t}$ coincides with the $\sigma$-algebra generated by the sets

$$
\begin{gathered}
E_{1} \times\left\{w \in C\left(\mathbb{R}_{+}, \mathbb{R}^{r}\right):\left(w\left(t_{1,1} \wedge s\right), \ldots, w\left(t_{1, n_{1}} \wedge s\right)\right) \in E_{2}\right\} \\
\times\left\{\pi \in M\left(\mathbb{R}_{+} \times U\right):\left(\pi\left(\left[0, t_{2,1} \wedge s\right] \times B_{1}\right), \ldots,\right.\right. \\
\left.\left.\pi\left(\left[0, t_{2, n_{2}} \wedge s\right] \times B_{n_{2}}\right)\right) \in E_{3}\right\} \\
\times\left\{y^{(1)} \in D\left(\mathbb{R}_{+}, \mathbb{R}^{d}\right):\left(y^{(1)}\left(t_{3,1} \wedge s\right), \ldots,\right.\right. \\
\times\left\{y^{(2)} \in D\left(\mathbb{R}_{+}, \mathbb{R}^{d}\right):\left(y^{(2)}\left(t_{4, n_{3}} \wedge s\right)\right) \in E_{4}\right\} \\
\left.\left.y^{(2)}\left(t_{4, n_{4}} \wedge s\right)\right) \in E_{5}\right\}
\end{gathered}
$$

for $s \in[0, t], t_{i, j} \in \mathbb{R}_{+}, i \in\{1,2,3,4\}, j \in\left\{1, \ldots, n_{i}\right\}$, $B_{1}, \ldots, B_{n_{2}} \in \mathscr{B}(U), E_{1} \in \mathscr{B}\left(\mathbb{R}^{d}\right), E_{2} \in \mathscr{B}\left(\mathbb{R}^{r n_{1}}\right), E_{3} \in$ $\mathscr{B}\left([0, \infty]^{n_{2}}\right), E_{4} \in \mathscr{B}\left(\mathbb{R}^{d n_{3}}\right)$, and $E_{5} \in \mathscr{B}\left(\mathbb{R}^{d n_{4}}\right)$. Since, for any stochastic process $\left(\xi_{t}\right)_{t \in \mathbb{R}_{+}}$,

$$
\begin{aligned}
& \sigma\left(\xi_{t}: t \in[0, s]\right) \\
& =\sigma\left(\left(\xi_{t_{1}}, \ldots, \xi_{t_{n}}\right): t_{i} \in[0, s], i \in\{1, \ldots, n\}, n \in \mathbb{N}\right), \\
& s \in \mathbb{R}_{+},
\end{aligned}
$$

we get $\widehat{\mathscr{G}}_{t}=\mathscr{G}_{t}, t \in \mathbb{R}_{+}$.

The $\pi$-coordinate process on $\Omega$ induces a point process $p_{\pi}$ on $U$ with characteristic measure $m$ in a natural way, since, as it was recalled, there is a bijection between the set of point functions on $U$ and the set of point measures $\pi$ on $\mathbb{R}_{+} \times U$ with $\pi(\{0\} \times U)=0$ and $\pi(\{t\} \times U) \leqslant 1, t \in \mathbb{R}_{++}$, and

$$
\begin{aligned}
& \mathbb{P}_{1,2}\left(\left\{\left(\mathbf{x}, w, \pi, y^{(1)}, y^{(2)}\right) \in \Omega:\right.\right. \\
& \left.\left.\quad \pi(\{0\} \times U)=0, \pi(\{t\} \times U) \leqslant 1, t \in \mathbb{R}_{++}\right\}\right)=1,
\end{aligned}
$$

which follows from (34) using that $P_{U, m}$ is the distribution on $\left(M\left(\mathbb{R}_{+} \times U\right), \mathscr{M}\left(\mathbb{R}_{+} \times U\right)\right)$ of a stationary Poisson point process on $U$ with characteristic measure $m$ implying that

$$
\begin{aligned}
P_{U, m}\left(\left\{\pi \in M\left(\mathbb{R}_{+} \times U\right):\right.\right. & \\
& \left.\left.\pi(\{0\} \times U)=0, \pi(\{t\} \times U) \leqslant 1, t \in \mathbb{R}_{++}\right\}\right)=1 .
\end{aligned}
$$

Next we check that $\left(\Omega, \mathscr{F},\left(\mathscr{F}_{t}\right)_{t \in \mathbb{R}_{+}}, \mathbb{P}_{1,2}, w, p_{\pi},(\mathbf{x}+\right.$ $\left.\left.y_{t}^{(i)}\right)_{t \in \mathbb{R}_{+}}\right), i \in\{1,2\}$, are weak solutions of the $\operatorname{SDE}(1)$ with the same initial distribution $n$. Using the definitions of $P_{i}$, $i \in\{1,2\}, \mathbb{P}_{1,2},(\mathrm{R} 1)$ and (R3) we get

$$
\begin{aligned}
\mathbb{P}_{1,2} & {\left[\omega=\left(\mathbf{x}, w, \pi, y^{(1)}, y^{(2)}\right) \in \Omega:\left(\mathbf{x}, w, \pi, y^{(i)}\right) \in A\right] } \\
& =\mathbb{P}^{(i)}\left[\left(\mathbf{X}_{0}^{(i)}, \mathbf{W}^{(i)}, p^{(i)}, \mathbf{Y}^{(i)}\right) \in A\right]
\end{aligned}
$$

for all $A \in \mathscr{B}(\Theta)$ and $i \in\{1,2\}$. Indeed, with $i=1, G \in$ $\mathscr{B}\left(\mathbb{R}^{d}\right) \otimes \mathscr{C}\left(\mathbb{R}_{+}, \mathbb{R}^{r}\right) \otimes \mathscr{M}\left(\mathbb{R}_{+} \times U\right)$ and $F \in \mathscr{D}\left(\mathbb{R}_{+}, \mathbb{R}^{d}\right)$, by Fubini theorem,

$$
\begin{aligned}
& \mathbb{P}_{1,2}\left[\omega=\left(\mathbf{x}, w, \pi, y^{(1)}, y^{(2)}\right) \in \Omega:\left(\mathbf{x}, w, \pi, y^{(1)}\right) \in G \times F\right] \\
& =\int_{\left\{\omega \in \Omega:\left(\mathbf{x}, w, \pi, y^{(1)}\right) \in G \times F\right\}} Q_{1}\left(\mathbf{x}, w, \pi, \mathrm{d} y^{(1)}\right) Q_{2}\left(\mathbf{x}, w, \pi, \mathrm{d} y^{(2)}\right) \\
& \qquad n(\mathrm{~d} \mathbf{x}) P_{\mathbf{W}, r}(\mathrm{~d} w) P_{U, m}(\mathrm{~d} \pi) \\
& =\int_{G} Q_{1}(\mathbf{x}, w, \pi, F) Q_{2}\left(\mathbf{x}, w, \pi, D\left(\mathbb{R}_{+}, \mathbb{R}^{d}\right)\right) \\
& =\int_{G} Q_{1}(\mathbf{x}, w, \pi, F) n(\mathrm{~d} \mathbf{x}) P_{\mathbf{W}, r}(\mathrm{~d} w) P_{U, m}(\mathrm{~d} \pi)=P_{1}(G \times F) \\
& =\mathbb{P}^{(1)}\left[\left(\mathbf{X}_{0}^{(1)}, \mathbf{W}^{(1)}, p^{(1)}, \mathbf{Y}^{(1)}\right) \in G \times F\right] .
\end{aligned}
$$

So the distribution of $\left(\mathbf{x}+y^{(i)}, w, p_{\pi}\right)$ under $\mathbb{P}_{1,2}$ is the same as the distribution of $\left(\mathbf{X}_{0}^{(i)}+\mathbf{Y}^{(i)}, \mathbf{W}^{(i)}, p^{(i)}\right)=\left(\mathbf{X}^{(i)}, \mathbf{W}^{(i)}, p^{(i)}\right)$ under $\mathbb{P}^{(i)}$. Due to the definition of a weak solution, under $\mathbb{P}^{(i)}, \mathbf{W}^{(i)}$ is an $r$-dimensional standard $\left(\mathscr{F}_{t}^{(i)}\right)_{t \in \mathbb{R}_{+}}$-Brownian motion, and $p^{(i)}$ is a stationary $\left(\mathscr{F}_{t}^{(i)}\right)_{t \in \mathbb{R}_{+}}$-Poisson point process on $U$ with characteristic measure $m$. Consequently, by the definition of $\left(\mathscr{G}_{t}\right)_{t \in \mathbb{R}_{+}}$(which is nothing else but the natural filtration corresponding to the coordinate processes), under $\mathbb{P}_{1,2}$, the $w$-coordinate process is an $r$-dimensional standard $\left(\mathscr{G}_{t}\right)_{t \in \mathbb{R}_{+}}$-Brownian motion, the process $p_{\pi}$ is a stationary $\left(\mathscr{G}_{t}\right)_{t \in \mathbb{R}_{+}}$-Poisson point process on $U$ with characteristic measure $m$, and $\left(\mathbf{x}+y_{t}^{(i)}\right)_{t \in \mathbb{R}_{+}}$is $\left(\mathscr{G}_{t}\right)_{t \in \mathbb{R}_{+}}$-adapted, $i \in\{1,2\}$. Further, the same is true if we replace the filtration $\left(\mathscr{G}_{t}\right)_{t \in \mathbb{R}_{+}}$by $\left(\mathscr{F}_{t}\right)_{t \in \mathbb{R}_{+}}$; see, Lemma A.5. Note also that the filtration $\left(\mathscr{F}_{t}\right)_{t \in \mathbb{R}_{+}}$satisfies the usual conditions. All in all, for each $i \in\{1,2\}$, the tuple $\left(\Omega, \mathscr{F},\left(\mathscr{F}_{t}\right)_{t \in \mathbb{R}_{+}}, \mathbb{P}_{1,2}, w, p_{\pi},(\mathbf{x}+\right.$ $\left.y_{t}^{(i)}\right)_{t \in \mathbb{R}_{+}}$) satisfies (D1)-(D3).

Hence it remains to check that, for each $i \in\{1,2\}$, the tuple $\left(\Omega, \mathscr{F},\left(\mathscr{F}_{t}\right)_{t \in \mathbb{R}_{+}}, \mathbb{P}_{1,2}, w, p_{\pi},\left(\mathbf{x}+y_{t}^{(i)}\right)_{t \in \mathbb{R}_{+}}\right)$satisfies (D4). 
For each $i \in\{1,2\}$, let us apply Lemma A.4 with the following choices:

$$
\begin{gathered}
\left(\Omega^{(i)}, \mathscr{F}^{(i)},\left(\mathscr{F}_{t}^{(i)}\right)_{t \in \mathbb{R}_{+}}, \mathbb{P}^{(i)}, \mathbf{W}^{(i)}, p^{(i)}, \mathbf{X}^{(i)}\right), \\
\left(\Omega, \mathscr{F},\left(\mathscr{F}_{t}\right)_{t \in \mathbb{R}_{+}}, \mathbb{P}_{1,2}, w, p_{\pi},\left(\mathbf{x}+y_{t}^{(i)}\right)_{t \in \mathbb{R}_{+}}\right) .
\end{gathered}
$$

Since $\left(\Omega^{(i)}, \mathscr{F}^{(i)},\left(\mathscr{F}_{t}^{(i)}\right)_{t \in \mathbb{R}_{+}}, \mathbb{P}^{(i)}, \mathbf{W}^{(i)}, p^{(i)}, \mathbf{X}^{(i)}\right)$ is a weak solution of the SDE (1) with initial distribution $n$, the tuple $\left(\Omega^{(i)}, \mathscr{F}^{(i)},\left(\mathscr{F}_{t}^{(i)}\right)_{t \in \mathbb{R}_{+}}, \mathbb{P}^{(i)}, \mathbf{W}^{(i)}, p^{(i)}, \mathbf{X}^{(i)}\right)$ satisfies (D1)-(D4). Further, as it was explained before, the tuple $\left(\Omega, \mathscr{F},\left(\mathscr{F}_{t}\right)_{t \in \mathbb{R}_{+}}\right.$, $\left.\mathbb{P}_{1,2}, w, p_{\pi},\left(\mathbf{x}+y_{t}^{(i)}\right)_{t \in \mathbb{R}_{+}}\right)$satisfies (D1)-(D3), the process $\left(\mathbf{x}+y_{t}^{(i)}\right)_{t \in \mathbb{R}_{+}}$is adapted to the filtration $\left(\mathscr{F}_{t}\right)_{t \in \mathbb{R}_{+}}$, and the distribution of $\left(\mathbf{X}^{(i)}, \mathbf{W}^{(i)}, p^{(i)}\right)$ under $\mathbb{P}^{(i)}$ is the same as the distribution of $\left(\mathbf{x}+y^{(i)}, w, p_{\pi}\right)$ under $\mathbb{P}_{1,2}$. Then Lemma A.4 yields that the tuple $\left(\Omega, \mathscr{F},\left(\mathscr{F}_{t}\right)_{t \in \mathbb{R}_{+}}, \mathbb{P}_{1,2}, w, p_{\pi},\left(\mathbf{x}+y_{t}^{(i)}\right)_{t \in \mathbb{R}_{+}}\right)$ satisfies (D4)(a)-(d) and the distribution of

$$
\begin{aligned}
& \left(\mathbf{X}_{t}^{(i)}-\mathbf{X}_{0}^{(i)}-\int_{0}^{t} b\left(s, \mathbf{X}_{s}^{(i)}\right) \mathrm{d} s-\int_{0}^{t} \sigma\left(s, \mathbf{X}_{s}^{(i)}\right) \mathrm{d} \mathbf{W}_{s}^{(i)}\right. \\
& \quad-\int_{0}^{t} \int_{U_{0}} f\left(s, \mathbf{X}_{s-}^{(i)}, u\right) \widetilde{N}^{(i)}(\mathrm{d} s, \mathrm{~d} u) \\
& \left.\quad-\int_{0}^{t} \int_{U_{1}} g\left(s, \mathbf{X}_{s-}^{(i)}, u\right) N^{(i)}(\mathrm{d} s, \mathrm{~d} u)\right)_{t \in \mathbb{R}_{+}}
\end{aligned}
$$

on $\left(D\left(\mathbb{R}_{+}, \mathbb{R}^{d}\right), \mathscr{D}\left(\mathbb{R}_{+}, \mathbb{R}^{d}\right)\right)$ under $\mathbb{P}^{(i)}$ is the same as the distribution of

$$
\begin{aligned}
& \left(y_{t}^{(i)}-y_{0}^{(i)}-\int_{0}^{t} b\left(s, \mathbf{x}+y_{s}^{(i)}\right) \mathrm{d} s\right. \\
& -\int_{0}^{t} \sigma\left(s, \mathbf{x}+y_{s}^{(i)}\right) \mathrm{d} w_{s} \\
& -\int_{0}^{t} \int_{U_{0}} f\left(s, \mathbf{x}+y_{s-}^{(i)}, u\right) \widetilde{N}_{\pi}(\mathrm{d} s, \mathrm{~d} u) \\
& \left.-\int_{0}^{t} \int_{U_{1}} g\left(s, \mathbf{x}+y_{s-}^{(i)}, u\right) N_{\pi}(\mathrm{d} s, \mathrm{~d} u)\right)_{t \in \mathbb{R}_{+}}
\end{aligned}
$$

on $\left(D\left(\mathbb{R}_{+}, \mathbb{R}^{d}\right), \mathscr{D}\left(\mathbb{R}_{+}, \mathbb{R}^{d}\right)\right)$ under $\mathbb{P}_{1,2}$, where $N_{\pi}(\mathrm{d} s, \mathrm{~d} u)$ is the counting measure of $p_{\pi}$ on $\mathbb{R}_{+} \times U$, and $\widetilde{N}_{\pi}(\mathrm{d} s, \mathrm{~d} u):=$ $N_{\pi}(\mathrm{d} s, \mathrm{~d} u)-\mathrm{d} s m(\mathrm{~d} u)$. Using also that for each $i \in\{1,2\}$, the first process and the identically 0 process are indistinguishable (since the SDE (1) holds $\mathbb{P}^{(i)}$-a.s. for $\left.\left(\mathbf{X}_{t}^{(i)}\right)_{t \in \mathbb{R}_{+}}\right)$, we obtain that the tuple $\left(\Omega, \mathscr{F},\left(\mathscr{F}_{t}\right)_{t \in \mathbb{R}_{+}}, \mathbb{P}_{1,2}, w, p_{\pi},\left(\mathbf{x}+y_{t}^{(i)}\right)_{t \in \mathbb{R}_{+}}\right)$satisfies (D4), as desired. It is worth mentioning that this is the place where we use that the filtration $\left(\mathscr{F}_{t}\right)_{t \in \mathbb{R}_{+}}$satisfies the usual conditions in order to ensure that the second process above has a càdlàg modification; see Remark 4 . The filtrations $\left(\mathscr{G}_{t}\right)_{t \in \mathbb{R}_{+}}$and $\left(\widetilde{\mathscr{G}}_{t}\right)_{t \in \mathbb{R}_{+}}$do not necessarily satisfy the usual conditions; this is the reason for introducing the filtration $\left(\mathscr{F}_{t}\right)_{t \in \mathbb{R}_{+}}$.
We have $\mathbb{P}_{1,2}\left(\mathbf{x}+y_{0}^{(1)}=\mathbf{x}+y_{0}^{(2)}\right)=1$, because, by (45), $\mathbb{P}_{1,2}\left(y_{0}^{(i)}=\mathbf{0}\right)=\mathbb{P}^{(i)}\left(\mathbf{Y}_{0}^{(i)}=\mathbf{0}\right)=1, i \in\{1,2\}$. Since $(\Omega$, $\left.\mathscr{F},\left(\mathscr{F}_{t}\right)_{t \in \mathbb{R}_{+}}, \mathbb{P}_{1,2}, w, p_{\pi},\left(\mathbf{x}+y_{t}^{(i)}\right)_{t \in \mathbb{R}_{+}}\right), i \in\{1,2\}$, are weak solutions of the SDE (1) with the same initial distribution $n$, and $\mathbb{P}_{1,2}\left(\mathbf{x}+y_{0}^{(1)}=\mathbf{x}+y_{0}^{(2)}\right)=1$, pathwise uniqueness implies $\mathbb{P}_{1,2}\left(\mathbf{x}+y_{t}^{(1)}=\mathbf{x}+y_{t}^{(2)}\right.$ for all $\left.t \in \mathbb{R}_{+}\right)=1$, or equivalently,

$$
\mathbb{P}_{1,2}\left[\omega=\left(\mathbf{x}, w, \pi, y^{(1)}, y^{(2)}\right) \in \Omega: y^{(1)}=y^{(2)}\right]=1,
$$

hence, applying (45),

$$
\begin{aligned}
& \mathbb{P}^{(1)}\left[\left(\mathbf{X}_{0}^{(1)}, \mathbf{W}^{(1)}, p^{(1)}, \mathbf{Y}^{(1)}\right) \in A\right] \\
& =\mathbb{P}_{1,2}\left[\omega=\left(\mathbf{x}, w, \pi, y^{(1)}, y^{(2)}\right) \in \Omega:\left(\mathbf{x}, w, \pi, y^{(1)}\right) \in A\right] \\
& =\mathbb{P}_{1,2}\left[\omega=\left(\mathbf{x}, w, \pi, y^{(1)}, y^{(2)}\right) \in \Omega:\left(\mathbf{x}, w, \pi, y^{(2)}\right) \in A\right] \\
& =\mathbb{P}^{(2)}\left[\left(\mathbf{X}_{0}^{(2)}, \mathbf{W}^{(2)}, p^{(2)}, \mathbf{Y}^{(2)}\right) \in A\right]
\end{aligned}
$$

for all $A \in \mathscr{B}(\Theta)$. Since $\mathbf{X}^{(i)}=\mathbf{X}_{0}^{(i)}+\mathbf{Y}^{(i)}, i \in\{1,2\}$, and the mapping $\mathbb{R}^{d} \times D\left(\mathbb{R}_{+}, \mathbb{R}^{d}\right) \ni\left(\mathbf{x}_{0}, \mathbf{y}\right) \mapsto \mathbf{x}_{0}+\mathbf{y} \in D\left(\mathbb{R}_{+}, \mathbb{R}^{d}\right)$ is continuous (see, e.g., Jacod and Shiryaev [17, Proposition VI.1.23]), we have

$$
\mathbb{P}^{(1)}\left[\mathbf{X}^{(1)} \in \widetilde{A}\right]=\mathbb{P}^{(2)}\left[\mathbf{X}^{(2)} \in \widetilde{A}\right], \quad \widetilde{A} \in \mathscr{D}\left(\mathbb{R}_{+}, \mathbb{R}^{d}\right),
$$

and then we obtain uniqueness in the sense of probability law.

\section{Precise Formulation and Proof of Theorem 2}

Our first result is a counterpart of Lemma 1.1 in Chapter IV in Ikeda and Watanabe [20] for stochastic differential equations with jumps, compare also with Situ [11, page 106, Fact A].

Lemma 12. If $\left(\Omega, \mathscr{F},\left(\mathscr{F}_{t}\right)_{t \in \mathbb{R}_{+}}, \mathbb{P}, \mathbf{W}, p, \mathbf{X}\right)$ is a weak solution of the $S D E$ (1) with initial distribution $n$ on $\left(\mathbb{R}^{d}, \mathscr{B}\left(\mathbb{R}^{d}\right)\right)$, then for every fixed $t \in \mathbb{R}_{+}$and $F \in \mathscr{D}_{t}\left(\mathbb{R}_{+}, \mathbb{R}^{d}\right)$, the mapping

$\mathbb{R}^{d} \times C\left(\mathbb{R}_{+}, \mathbb{R}^{r}\right) \times M\left(\mathbb{R}_{+} \times U\right) \ni(\mathbf{x}, w, \pi) \longmapsto Q(\mathbf{x}, w, \pi, F)$

is $\widehat{\mathscr{B}}_{t} / \mathscr{B}([0,1])$-measurable, where $\widehat{\mathscr{B}}_{t}$ denotes the completion of $\mathscr{B}\left(\mathbb{R}^{d}\right) \otimes \mathscr{C}_{t}\left(\mathbb{R}_{+}, \mathbb{R}^{r}\right) \otimes \mathscr{M}_{t}\left(\mathbb{R}_{+} \times U\right)$ by the null sets of $n \times$ $P_{\mathrm{W}, r} \times P_{U, m}$ from $\mathscr{B}\left(\mathbb{R}^{d}\right) \otimes \mathscr{C}\left(\mathbb{R}_{+}, \mathbb{R}^{r}\right) \otimes \mathscr{M}\left(\mathbb{R}_{+} \times U\right)$.

Proof. Consider the regular conditional probability

$$
\begin{aligned}
Q_{t}: \mathbb{R}^{d} & \times C\left(\mathbb{R}_{+}, \mathbb{R}^{r}\right) \times M\left(\mathbb{R}_{+} \times U\right) \\
& \times \mathscr{D}_{t}\left(\mathbb{R}_{+}, \mathbb{R}^{d}\right) \longrightarrow[0,1]
\end{aligned}
$$

for $\mathscr{D}_{t}\left(\mathbb{R}_{+}, \mathbb{R}^{d}\right)$ given $\left(x, \varphi_{t}(w), \psi_{t}(\pi)\right)$, where, for each $t \in$ $\mathbb{R}_{+}$, the stopped mapping $\varphi_{t}: C\left(\mathbb{R}_{+}, \mathbb{R}^{r}\right) \rightarrow C\left(\mathbb{R}_{+}, \mathbb{R}^{r}\right)$ is defined in $(9)$, and $\psi_{t}: M\left(\mathbb{R}_{+} \times U\right) \rightarrow M\left(\mathbb{R}_{+} \times U\right), \psi_{t}(\pi):=$ $\left.\pi\right|_{[0, t] \times U}, \pi \in M\left(\mathbb{R}_{+} \times U\right)$; that is, $\psi_{t}(\pi)$ denotes the restriction of $\pi$ onto $[0, t] \times U$. The mapping $Q_{t}$ enjoy properties analogous to (R1)-(R3). Namely, 
$(\widetilde{\mathrm{R} 1})$ for each $\mathbf{x} \in \mathbb{R}^{d}, w \in C\left(\mathbb{R}_{+}, \mathbb{R}^{r}\right)$, and $\pi \in M\left(\mathbb{R}_{+} \times U\right)$, the set function $\mathscr{D}_{t}\left(\mathbb{R}_{+}, \mathbb{R}^{d}\right) \ni F \mapsto Q_{t}(\mathbf{x}, w, \pi, F)$ is a probability measure on $\left(D\left(\mathbb{R}_{+}, \mathbb{R}^{d}\right), \mathscr{D}_{t}\left(\mathbb{R}_{+}, \mathbb{R}^{d}\right)\right)$;

$(\widetilde{\mathrm{R} 2})$ for each $F \in \mathscr{D}_{t}\left(\mathbb{R}_{+}, \mathbb{R}^{d}\right)$, the mapping $\mathbb{R}^{d} \times C\left(\mathbb{R}_{+}\right.$, $\left.\mathbb{R}^{r}\right) \times M\left(\mathbb{R}_{+} \times U\right) \ni(\mathbf{x}, w, \pi) \mapsto Q_{t}(\mathbf{x}, w, \pi, F)$ is $\mathscr{B}\left(\mathbb{R}^{d}\right) \otimes \mathscr{C}_{t}\left(\mathbb{R}_{+}, \mathbb{R}^{r}\right) \otimes \mathscr{M}_{t}\left(\mathbb{R}_{+} \times U\right) / \mathscr{B}([0,1])$-measurable;

$(\widetilde{\mathrm{R} 3})$ for every $G \in \mathscr{B}\left(\mathbb{R}^{d}\right) \otimes \mathscr{C}_{t}\left(\mathbb{R}_{+}, \mathbb{R}^{r}\right) \otimes \mathscr{M}_{t}\left(\mathbb{R}_{+} \times U\right)$ and $F \in \mathscr{D}_{t}\left(\mathbb{R}_{+}, \mathbb{R}^{d}\right)$,

$$
P(G \times F)=\int_{G} Q_{t}(\mathbf{x}, w, \pi, F) n(\mathrm{~d} \mathbf{x}) P_{\mathbf{W}, r}(\mathrm{~d} w) P_{U, m}(\mathrm{~d} \pi),
$$

where the probability measure $P$ is defined in (28).

In order to prove the statement, it suffices to check that

$$
\begin{aligned}
& Q(\mathbf{x}, w, \pi, F)=Q_{t}(\mathbf{x}, w, \pi, F) \\
& \text { for } n \times P_{\mathbf{W}, r} \times P_{U, m} \text {-a.e. } \quad(\mathbf{x}, w, \pi) .
\end{aligned}
$$

Indeed, then $\left(n \times P_{\mathrm{W}, r} \times P_{U, m}\right)(N)=0$ for

$$
\begin{gathered}
N:=\left\{(\mathbf{x}, w, \pi) \in \mathbb{R}^{d} \times C\left(\mathbb{R}_{+}, \mathbb{R}^{r}\right) \times M\left(\mathbb{R}_{+} \times U\right):\right. \\
\left.Q(\mathbf{x}, w, \pi, F) \neq Q_{t}(\mathbf{x}, w, \pi, F)\right\} \\
\in \mathscr{B}\left(\mathbb{R}^{d}\right) \otimes \mathscr{C}\left(\mathbb{R}_{+}, \mathbb{R}^{r}\right) \otimes \mathscr{M}\left(\mathbb{R}_{+} \times U\right),
\end{gathered}
$$

and what is more, $N \in \widehat{\mathscr{B}}_{t}$, since

$$
\widehat{\mathscr{B}}_{t}=\sigma\left(\mathscr{B}\left(\mathbb{R}^{d}\right) \otimes \mathscr{C}_{t}\left(\mathbb{R}_{+}, \mathbb{R}^{r}\right) \otimes \mathscr{M}_{t}\left(\mathbb{R}_{+} \times U\right) \cup \mathcal{N}\right),
$$

where

$$
\begin{gathered}
\mathcal{N}:=\left\{A \subset \mathbb{R}^{d} \times C\left(\mathbb{R}_{+}, \mathbb{R}^{r}\right) \times M\left(\mathbb{R}_{+} \times U\right):\right. \\
\exists B \in \mathscr{B}\left(\mathbb{R}^{d}\right) \otimes \mathscr{C}\left(\mathbb{R}_{+}, \mathbb{R}^{r}\right) \otimes \mathscr{M}\left(\mathbb{R}_{+} \times U\right) \\
\text { with } \left.A \subset B,\left(n \times P_{\mathrm{W}, r} \times P_{U, m}\right)(B)=0\right\},
\end{gathered}
$$

and $N \in \mathscr{N}$. Hence, for all $E \in \mathscr{B}([0,1])$,

$$
\begin{gathered}
\left\{(\mathbf{x}, w, \pi) \in \mathbb{R}^{d} \times C\left(\mathbb{R}_{+}, \mathbb{R}^{r}\right) \times M\left(\mathbb{R}_{+} \times U\right):\right. \\
Q(\mathbf{x}, w, \pi, F) \in E\}=A_{1} \cup A_{2},
\end{gathered}
$$

where

$$
\begin{aligned}
& A_{1}:=\left\{(\mathbf{x}, w, \pi) \in \mathbb{R}^{d} \times C\left(\mathbb{R}_{+}, \mathbb{R}^{r}\right) \times M\left(\mathbb{R}_{+} \times U\right):\right. \\
& Q(\mathbf{x}, w, \pi, F) \in E, \\
&\left.Q(\mathbf{x}, w, \pi, F)=Q_{t}(\mathbf{x}, w, \pi, F)\right\} \\
&=\left\{(\mathbf{x}, w, \pi) \in \mathbb{R}^{d} \times C\left(\mathbb{R}_{+}, \mathbb{R}^{r}\right) \times M\left(\mathbb{R}_{+} \times U\right):\right. \\
&\left.Q_{t}(\mathbf{x}, w, \pi, F) \in E\right\}
\end{aligned}
$$

$$
\begin{gathered}
\cap\left\{(\mathbf{x}, w, \pi) \in \mathbb{R}^{d} \times C\left(\mathbb{R}_{+}, \mathbb{R}^{r}\right) \times M\left(\mathbb{R}_{+} \times U\right):\right. \\
\left.Q(\mathbf{x}, w, \pi, F)=Q_{t}(\mathbf{x}, w, \pi, F)\right\},
\end{gathered}
$$

$$
\begin{aligned}
A_{2}:=\left\{(\mathbf{x}, w, \pi) \in \mathbb{R}^{d} \times C\left(\mathbb{R}_{+}, \mathbb{R}^{r}\right) \times M\left(\mathbb{R}_{+} \times U\right):\right. \\
\left.Q(\mathbf{x}, w, \pi, F) \in E, Q(\mathbf{x}, w, \pi, F) \neq Q_{t}(\mathbf{x}, w, \pi, F)\right\} .
\end{aligned}
$$

Here $A_{1} \in \widehat{\mathscr{B}}_{t}$, since, by $(\widetilde{\mathrm{R} 2})$, the set

$$
\begin{gathered}
\left\{(\mathbf{x}, w, \pi) \in \mathbb{R}^{d} \times C\left(\mathbb{R}_{+}, \mathbb{R}^{r}\right) \times M\left(\mathbb{R}_{+} \times U\right):\right. \\
\left.Q_{t}(\mathbf{x}, w, \pi, F) \in E\right\}
\end{gathered}
$$

is in $\mathscr{B}\left(\mathbb{R}^{d}\right) \otimes \mathscr{C}_{t}\left(\mathbb{R}_{+}, \mathbb{R}^{r}\right) \otimes \mathscr{M}_{t}\left(\mathbb{R}_{+} \times U\right) \subset \widehat{\mathscr{B}}_{t}$, and

$$
\begin{aligned}
& \left\{(\mathbf{x}, w, \pi) \in \mathbb{R}^{d} \times C\left(\mathbb{R}_{+}, \mathbb{R}^{r}\right) \times M\left(\mathbb{R}_{+} \times U\right):\right. \\
& \left.Q(\mathbf{x}, w, \pi, F)=Q_{t}(\mathbf{x}, w, \pi, F)\right\} \\
& \quad=\mathbb{R}^{d} \times C\left(\mathbb{R}_{+}, \mathbb{R}^{r}\right) \times M\left(\mathbb{R}_{+} \times U\right) \backslash N \in \widehat{\mathscr{B}}_{t} .
\end{aligned}
$$

Further, $A_{2} \subset N \in \mathscr{B}\left(\mathbb{R}^{d}\right) \otimes \mathscr{C}\left(\mathbb{R}_{+}, \mathbb{R}^{r}\right) \otimes \mathscr{M}\left(\mathbb{R}_{+} \times U\right)$ and $\left(n \times P_{\mathrm{W}, r} \times P_{U, m}\right)(N)=0$ imply $A_{2} \in \mathcal{N} \subset \widehat{\mathscr{B}}_{t}$.

Unfortunately, (57) does not follow from the comparison of (R3) with $(\widetilde{\mathrm{R} 3})$, since still we do not know whether the function $(\mathbf{x}, w, \pi) \mapsto Q(\mathbf{x}, w, \pi, F)$ is $\mathscr{B}\left(\mathbb{R}^{d}\right) \otimes \mathscr{C}_{t}\left(\mathbb{R}_{+}, \mathbb{R}^{r}\right) \otimes$ $\mathscr{M}_{t}\left(\mathbb{R}_{+} \times U\right) / \mathscr{B}([0,1])$-measurable. In order to show (57), it suffices to check that $(\widetilde{\mathrm{R} 3})$ is valid for every $G \in \mathscr{B}\left(\mathbb{R}^{d}\right) \otimes$ $\mathscr{C}\left(\mathbb{R}_{+}, \mathbb{R}^{r}\right) \otimes \mathscr{M}\left(\mathbb{R}_{+} \times U\right)$. Indeed, then, by (R3),

$$
\begin{aligned}
\int_{G} Q & (\mathbf{x}, w, \pi, F) n(\mathrm{~d} \mathbf{x}) P_{\mathrm{W}, r}(\mathrm{~d} w) P_{U, m}(\mathrm{~d} \pi) \\
& =\int_{G} Q_{t}(\mathbf{x}, w, \pi, F) n(\mathrm{~d} \mathbf{x}) P_{\mathrm{W}, r}(\mathrm{~d} w) P_{U, m}(\mathrm{~d} \pi)
\end{aligned}
$$

for all $G \in \mathscr{B}\left(\mathbb{R}^{d}\right) \otimes \mathscr{C}\left(\mathbb{R}_{+}, \mathbb{R}^{r}\right) \otimes \mathscr{M}\left(\mathbb{R}_{+} \times U\right)$ and $F \in$ $\mathscr{D}_{t}\left(\mathbb{R}_{+}, \mathbb{R}^{d}\right)$, and hence, using also that the function $(\mathbf{x}, w$, $\pi) \mapsto Q_{t}(\mathbf{x}, w, \pi, F)$ is $\mathscr{B}\left(\mathbb{R}^{d}\right) \otimes \mathscr{C}\left(\mathbb{R}_{+}, \mathbb{R}^{r}\right) \otimes \mathscr{M}\left(\mathbb{R}_{+} \times\right.$ $U) / \mathscr{B}([0,1])$-measurable, by the uniqueness part of the Radon-Nikodým theorem, we have (57).

The class $\mathscr{G}$ of sets $G$ satisfying $(\widetilde{\mathrm{R} 3})$ is a Dynkin system; that is,

(i) $\mathbb{R}^{d} \times C\left(\mathbb{R}_{+}, \mathbb{R}^{r}\right) \times M\left(\mathbb{R}_{+} \times U\right) \in \mathscr{G}$, since $\mathbb{R}^{d} \times C\left(\mathbb{R}_{+}\right.$, $\left.\mathbb{R}^{r}\right) \times M\left(\mathbb{R}_{+} \times U\right) \in \mathscr{B}\left(\mathbb{R}^{d}\right) \otimes \mathscr{C}_{t}\left(\mathbb{R}_{+}, \mathbb{R}^{r}\right) \otimes \mathscr{M}_{t}\left(\mathbb{R}_{+} \times U\right)$ and one can apply $(\widetilde{\mathrm{R} 3})$.

(ii) If $G_{1}, G_{2} \in \mathscr{G}$ and $G_{1} \subset G_{2}$, then $G_{2} \backslash G_{1} \in \mathscr{G}$. Indeed,

$$
\begin{aligned}
& P\left(\left(G_{2} \backslash G_{1}\right) \times F\right) \\
& \quad=P\left(G_{2} \times F\right)-P\left(G_{1} \times F\right)
\end{aligned}
$$




$$
\begin{aligned}
& =\int_{G_{2}} Q_{t}(\mathbf{x}, w, \pi, F) n(\mathrm{~d} \mathbf{x}) P_{\mathrm{W}, r}(\mathrm{~d} w) P_{U, m}(\mathrm{~d} \pi) \\
& \quad-\int_{G_{1}} Q_{t}(\mathbf{x}, w, \pi, F) n(\mathrm{~d} \mathbf{x}) P_{\mathrm{W}, r}(\mathrm{~d} w) P_{U, m}(\mathrm{~d} \pi) \\
& =\int_{G_{2} \backslash G_{1}} Q_{t}(\mathbf{x}, w, \pi, F) n(\mathrm{~d} \mathbf{x}) P_{\mathrm{W}, r}(\mathrm{~d} w) P_{U, m}(\mathrm{~d} \pi) .
\end{aligned}
$$

(iii) If $\left(G_{n}\right)_{n \in \mathbb{N}} \subset \mathscr{G}$ and $G_{1} \subset G_{2} \subset \cdots$, then $\bigcup_{n=1}^{\infty} G_{n} \in \mathscr{G}$. Indeed, by the continuity of probability and dominated convergence theorem,

$$
\begin{aligned}
& P\left(\left(\bigcup_{n=1}^{\infty} G_{n}\right) \times F\right) \\
& =\lim _{n \rightarrow \infty} P\left(G_{n} \times F\right) \\
& =\lim _{n \rightarrow \infty} \int_{G_{n}} Q_{t}(\mathbf{x}, w, \pi, F) n(\mathrm{~d} \mathbf{x}) P_{\mathbf{W}, r}(\mathrm{~d} w) P_{U, m}(\mathrm{~d} \pi) \\
& =\lim _{n \rightarrow \infty} \int_{\mathbb{R}^{d} \times C\left(\mathbb{R}_{+}, \mathbb{R}^{r}\right) \times M\left(\mathbb{R}_{+} \times U\right)} Q_{t}(\mathbf{x}, w, \pi, F) \mathbb{1}_{G_{n}}(\mathbf{x}, w, \pi) \\
& =\int_{\bigcup_{n=1}^{\infty} G_{n}} Q_{t}(\mathbf{x}, w, \pi, F) n(\mathrm{~d} \mathbf{x}) P_{\mathbf{W}, r}(\mathrm{~d} w) P_{\mathrm{W}, r}(\mathrm{~d} w) P_{U, m}(\mathrm{~d} \pi)
\end{aligned}
$$

Consider the collection of sets of the form

$$
G=G_{1} \times\left(\varphi_{t}^{-1}\left(G_{2}\right) \cap \widetilde{\varphi}_{t}^{-1}\left(G_{3}\right)\right) \times\left(\psi_{t}^{-1}\left(G_{4}\right) \cap \widetilde{\psi}_{t}^{-1}\left(G_{5}\right)\right)
$$

for $G_{1} \in \mathscr{B}\left(\mathbb{R}^{d}\right), G_{2}, G_{3} \in \mathscr{C}\left(\mathbb{R}_{+}, \mathbb{R}^{r}\right)$, and $G_{4}, G_{5} \in \mathscr{M}\left(\mathbb{R}_{+} \times\right.$ $U)$, where, for each $t \in \mathbb{R}_{+}, \varphi_{t}$ and $\psi_{t}$ are defined earlier, $\widetilde{\varphi}_{t}$ : $C\left(\mathbb{R}_{+}, \mathbb{R}^{r}\right) \rightarrow C\left(\mathbb{R}_{+}, \mathbb{R}^{r}\right)$ denotes the increment mapping $\left(\widetilde{\varphi}_{t}(w)\right)(s):=w(t+s)-w(t), w \in C\left(\mathbb{R}_{+}, \mathbb{R}^{r}\right), s \in \mathbb{R}_{+}$, and $\widetilde{\psi}_{t}:$ $M\left(\mathbb{R}_{+} \times U\right) \rightarrow M\left(\mathbb{R}_{+} \times U\right)$ denotes the increment mapping given by $\widetilde{\psi}_{t}(\pi)([0, s] \times B):=\pi([0, t+s] \times B)-\pi([0, t] \times B)$, $s \in \mathbb{R}_{+}, B \in \mathscr{B}(U)$. This collection of sets is closed under pairwise intersection and generates the $\sigma$-algebra $\mathscr{B}\left(\mathbb{R}^{d}\right) \otimes$ $\mathscr{C}\left(\mathbb{R}_{+}, \mathbb{R}^{r}\right) \otimes \mathscr{M}\left(\mathbb{R}_{+} \times U\right)$, since the collection of sets of the form $\left(\varphi_{t}^{-1}\left(G_{2}\right) \cap \tilde{\varphi}_{t}^{-1}\left(G_{3}\right)\right)$ with $G_{2}=\left\{w \in C\left(\mathbb{R}_{+}, \mathbb{R}^{r}\right)\right.$ : $\left.\left(w\left(t_{1}\right), \ldots, w\left(t_{n}\right)\right) \in A\right\}$ for $n \in \mathbb{N}, t \in \mathbb{R}_{+}, t_{1}, \ldots, t_{n} \in[0, t]$, $A \in \mathscr{B}\left(\mathbb{R}^{r n}\right)$, and $G_{3}=C\left(\mathbb{R}_{+}, \mathbb{R}^{r}\right)$ generates $\mathscr{C}\left(\mathbb{R}_{+}, \mathbb{R}^{r}\right)$ by (11), and the collection of sets of the form $\left(\psi_{t}^{-1}\left(G_{4}\right) \cap \widetilde{\psi}_{t}^{-1}\left(G_{5}\right)\right)$ with

$$
G_{4}=\left\{\pi \in M\left(\mathbb{R}_{+} \times U\right): \pi([0, t] \times B) \in A\right\}
$$

for $t \in \mathbb{R}_{+}, B \in \mathscr{B}(U), A \in \mathscr{B}([0, \infty])$, and $G_{5}=M\left(\mathbb{R}_{+} \times U\right)$ generates $\mathscr{M}\left(\mathbb{R}_{+} \times U\right)$ by (15). By the Dynkin system theorem (see, e.g., Karatzas and Shreve [21, Theorem 2.1.3]), $\mathscr{B}\left(\mathbb{R}^{d}\right) \otimes$ $\mathscr{C}\left(\mathbb{R}_{+}, \mathbb{R}^{r}\right) \otimes \mathscr{M}\left(\mathbb{R}_{+} \times U\right) \subset \mathscr{G}$ provided that we prove $(\widetilde{\mathrm{R} 3})$ for $G$ of the form (69). For such a $G$, by Fubini theorem, we have

$$
\begin{aligned}
& \int_{G} Q_{t}(\mathbf{x}, w, \pi, F) n(\mathrm{~d} \mathbf{x}) P_{\mathrm{W}, r}(\mathrm{~d} w) P_{U, m}(\mathrm{~d} \pi) \\
& =\int_{\psi_{t}^{-1}\left(G_{4}\right) \cap \widetilde{\psi}_{t}^{-1}\left(G_{5}\right)}\left(\int_{\varphi_{t}^{-1}\left(G_{2}\right) \cap \widetilde{\varphi}_{t}^{-1}\left(G_{3}\right)}\left(\int_{G_{1}} Q_{t}(\mathbf{x}, w, \pi, F) n(\mathrm{~d} \mathbf{x})\right)\right.
\end{aligned}
$$

$$
\left.P_{\mathrm{W}, r}(\mathrm{~d} w)\right) P_{U, m}(\mathrm{~d} \pi)
$$

$$
\begin{gathered}
=\mathbb{E}_{P_{\mathrm{W}, r} \times P_{U, m}}\left[\int_{G_{1}} Q_{t}(\mathbf{x}, w, \pi, F) n(\mathrm{~d} \mathbf{x}) \mathbb{1}_{\varphi_{t}^{-1}\left(G_{2}\right) \cap \tilde{\varphi}_{t}^{-1}\left(G_{3}\right)}(w)\right. \\
\left.\times \mathbb{1}_{\psi_{t}^{-1}\left(G_{4}\right) \cap \tilde{\psi}_{t}^{-1}\left(G_{5}\right)}(\pi)\right] \\
=\mathbb{E}_{P_{\mathrm{W}, r} \times P_{U, m}}\left[\mathbb { E } _ { P _ { \mathrm { W } , r } \times P _ { U , m } } \left[\int_{G_{1}} Q_{t}(\mathbf{x}, w, \pi, F) n(\mathrm{~d} \mathbf{x})\right.\right. \\
\times \mathbb{1}_{\varphi_{t}^{-1}\left(G_{2}\right)}(w) \mathbb{1}_{\tilde{\varphi}_{t}^{-1}\left(G_{3}\right)}(w) \\
\times \mathbb{1}_{\psi_{t}^{-1}\left(G_{4}\right)}(\pi) \mathbb{1}_{\widetilde{\psi}_{t}^{-1}\left(G_{5}\right)}(\pi) \mid \\
\left.\left.\mathscr{C}_{t}\left(\mathbb{R}_{+}, \mathbb{R}^{r}\right) \otimes \mathscr{M}_{t}\left(\mathbb{R}_{+} \times U\right)\right]\right]
\end{gathered}
$$

$$
\begin{aligned}
& =\mathbb{E}_{P_{\mathbf{W}, r} \times P_{U, m}}\left[\int_{G_{1}} Q_{t}(\mathbf{x}, w, \pi, F) n(\mathrm{~d} \mathbf{x})\right. \\
& \quad \times \mathbb{1}_{\varphi_{t}^{-1}\left(G_{2}\right)}(w) \mathbb{1}_{\psi_{t}^{-1}\left(G_{4}\right)}(\pi) \\
& \quad \times\left(P_{\mathbf{W}, r} \times P_{U, m}\right)\left(\tilde{\varphi}_{t}^{-1}\left(G_{3}\right) \times \tilde{\psi}_{t}^{-1}\left(G_{5}\right) \mid\right. \\
& \left.\left.\mathscr{C}_{t}\left(\mathbb{R}_{+}, \mathbb{R}^{r}\right) \otimes \mathscr{M}_{t}\left(\mathbb{R}_{+} \times U\right)\right)\right]
\end{aligned}
$$

$$
\begin{aligned}
& =\mathbb{E}_{P_{\mathbf{W}, r} \times P_{U, m}}\left[\int_{G_{1}} Q_{t}(\mathbf{x}, w, \pi, F) n(\mathrm{~d} \mathbf{x}) \mathbb{1}_{\varphi_{t}^{-1}\left(G_{2}\right)}(w) \mathbb{1}_{\psi_{t}^{-1}\left(G_{4}\right)}(\pi)\right. \\
& \left.\quad \times\left(P_{\mathbf{W}, r} \times P_{U, m}\right)\left(\widetilde{\varphi}_{t}^{-1}\left(G_{3}\right) \times \widetilde{\psi}_{t}^{-1}\left(G_{5}\right)\right)\right] \\
& =\int_{G_{1} \times \varphi_{t}^{-1}\left(G_{2}\right) \times \psi_{t}^{-1}\left(G_{4}\right)} Q_{t}(\mathbf{x}, w, \pi, F) n(\mathrm{~d} \mathbf{x}) P_{\mathbf{W}, r}(\mathrm{~d} w) P_{U, m}(\mathrm{~d} \pi) \\
& \quad \times\left(P_{\mathbf{W}, r} \times P_{U, m}\right)\left(\tilde{\varphi}_{t}^{-1}\left(G_{3}\right) \times \tilde{\psi}_{t}^{-1}\left(G_{5}\right)\right) \\
& =P\left[G_{1} \times \varphi_{t}^{-1}\left(G_{2}\right) \times \psi_{t}^{-1}\left(G_{4}\right) \times F\right] \\
& \quad \times\left(P_{\mathbf{W}, r} \times P_{U, m}\right)\left(\tilde{\varphi}_{t}^{-1}\left(G_{3}\right) \times \widetilde{\psi}_{t}^{-1}\left(G_{5}\right)\right) .
\end{aligned}
$$

The fourth equality above follows from the $\mathscr{C}_{t}\left(\mathbb{R}_{+}, \mathbb{R}^{r}\right) \otimes$ $\mathscr{M}_{t}\left(\mathbb{R}_{+} \times U\right) / \mathscr{B}([0,1])$-measurability of the function

$$
\begin{aligned}
C\left(\mathbb{R}_{+}, \mathbb{R}^{r}\right) \times M\left(\mathbb{R}_{+} \times U\right) \ni(w, \pi) \\
\longmapsto \int_{G_{1}} Q_{t}(\mathbf{x}, w, \pi, F) n(\mathrm{~d} \mathbf{x}),
\end{aligned}
$$


which is a consequence of $(\widetilde{\mathrm{R} 2})$ and Fubini theorem. The fifth equality above follows from the independence of $\widetilde{\varphi}_{t}^{-1}\left(G_{3}\right) \times$ $\widetilde{\psi}_{t}^{-1}\left(G_{5}\right)$ and $\mathscr{C}_{t}\left(\mathbb{R}_{+}, \mathbb{R}^{r}\right) \otimes \mathscr{M}_{t}\left(\mathbb{R}_{+} \times U\right)$ under the measure $P_{\mathrm{W}, r} \times P_{U, m} ;$ see, for example, Ikeda and Watanabe $[20$, Chapter 2, Theorems 6.4 and 6.5]. For the last equality above we used $(\widetilde{\mathrm{R} 3})$ and

$$
\begin{aligned}
G_{1} & \times \varphi_{t}^{-1}\left(G_{2}\right) \times \psi_{t}^{-1}\left(G_{4}\right) \times F \\
& \in \mathscr{B}\left(\mathbb{R}^{d}\right) \otimes \mathscr{C}_{t}\left(\mathbb{R}_{+}, \mathbb{R}^{r}\right) \otimes \mathscr{M}_{t}\left(\mathbb{R}_{+} \times U\right) \otimes \mathscr{D}_{t}\left(\mathbb{R}_{+}, \mathbb{R}^{d}\right) .
\end{aligned}
$$

By (28),

$$
\begin{aligned}
\left(P_{\mathbf{W}, r} \times P_{U, m}\right)\left(\widetilde{\varphi}_{t}^{-1}\left(G_{3}\right) \times \widetilde{\psi}_{t}^{-1}\left(G_{5}\right)\right) & \\
& =P\left[(\mathbf{x}, w, \pi, y) \in \Theta: \widetilde{\varphi}_{t}(w) \in G_{3}, \widetilde{\psi}_{t}(\pi) \in G_{5}\right] \\
& =\mathbb{P}\left[\widetilde{\varphi}_{t}(\mathbf{W}) \in G_{3}, \widetilde{\psi}_{t}(p) \in G_{5}\right], \\
P & {\left[G_{1} \times \varphi_{t}^{-1}\left(G_{2}\right) \times \psi_{t}^{-1}\left(G_{4}\right) \times F\right] } \\
& =\mathbb{P}\left[\mathbf{X}_{0} \in G_{1}, \varphi_{t}(\mathbf{W}) \in G_{2}, \psi_{t}(p) \in G_{4}, \mathbf{Y} \in F\right] .
\end{aligned}
$$

Therefore, if $G$ is of the form (69), then

$$
\begin{aligned}
\int_{G} Q_{t}(\mathbf{x}, w, \pi, F) n(\mathrm{~d} \mathbf{x}) P_{\mathbf{W}, r}(\mathrm{~d} w) P_{U, m}(\mathrm{~d} \pi) \\
=\mathbb{P}\left[\mathbf{X}_{0} \in G_{1}, \varphi_{t}(\mathbf{W}) \in G_{2}, \psi_{t}(p) \in G_{4}, \mathbf{Y} \in F\right] \\
\times \mathbb{P}\left[\widetilde{\varphi}_{t}(\mathbf{W}) \in G_{3}, \widetilde{\psi}_{t}(p) \in G_{5}\right] \\
=\mathbb{P}\left[\mathbf{X}_{0} \in G_{1}, \varphi_{t}(\mathbf{W}) \in G_{2}, \widetilde{\varphi}_{t}(\mathbf{W}) \in G_{3},\right. \\
\left.\quad \psi_{t}(p) \in G_{4}, \widetilde{\psi}_{t}(p) \in G_{5}, \mathbf{Y} \in F\right] \\
=\mathbb{P}\left[\left(\mathbf{X}_{0}, \mathbf{W}, p\right) \in G, \mathbf{Y} \in F\right] \\
=P[G \times F] .
\end{aligned}
$$

The second equality above follows from the independence of $\left\{\mathbf{X}_{0} \in G_{1}, \varphi_{t}(\mathbf{W}) \in G_{2}, \psi_{t}(p) \in G_{4}, \mathbf{Y} \in F\right\}$ and $\left\{\widetilde{\varphi}_{t}(\mathbf{W}) \in G_{3}\right.$, $\left.\widetilde{\psi}_{t}(p) \in G_{5}\right\}$ under the probability measure $\mathbb{P}$. This independence holds because

$$
\begin{aligned}
\left\{\mathbf{X}_{0} \in G_{1}, \varphi_{t}(\mathbf{W}) \in G_{2}, \psi_{t}(p) \in G_{4}, \mathbf{Y} \in F\right\} \\
=\left\{\mathbf{X}_{0} \in G_{1}, \varphi_{t}\left(\varphi_{t}(\mathbf{W})\right) \in G_{2}, \psi_{t}\left(\psi_{t}(p)\right) \in G_{4}, \mathbf{Y} \in F\right\} \\
=\left\{\mathbf{X}_{0} \in G_{1}, \varphi_{t}(\mathbf{W}) \in \varphi_{t}^{-1}\left(G_{2}\right), \psi_{t}(p) \in \psi_{t}^{-1}\left(G_{4}\right), \mathbf{Y} \in F\right\} \\
\quad \in \mathscr{F}_{t}
\end{aligned}
$$

and $\left\{\widetilde{\varphi}_{t}(\mathbf{W}) \in G_{3}, \widetilde{\psi}_{t}(p) \in G_{5}\right\}$ is independent of $\mathscr{F}_{t}$ under the probability measure $\mathbb{P}$; see, for example, Ikeda and Watanabe [20, Chapter II, Theorems 6.4 and 6.5]. The relationship (76) is valid since $\varphi_{t}^{-1}\left(G_{2}\right) \in \mathscr{C}_{t}\left(\mathbb{R}_{+}, \mathbb{R}^{r}\right), \psi_{t}^{-1}\left(G_{4}\right) \in \mathscr{M}_{t}\left(\mathbb{R}_{+} \times U\right)$, and $F \in \mathscr{D}_{t}\left(\mathbb{R}_{+}, \mathbb{R}^{d}\right)$, the mapping $\Omega \ni \omega \mapsto \varphi_{t}(\mathbf{W}(\omega))$ is $\mathscr{F}_{t} / \mathscr{C}_{t}\left(\mathbb{R}_{+}, \mathbb{R}^{r}\right)$-measurable, and the mapping $\Omega \ni \omega \mapsto$ $\psi_{t}(p(\omega))$ is $\mathscr{F}_{t} / \mathscr{M}_{t}\left(\mathbb{R}_{+} \times U\right)$-measurable, because the processes $\mathbf{W}$ and $p$ are $\left(\mathscr{F}_{t}\right)_{t \in \mathbb{R}_{+}}$-adapted.
Remark 13. The filtration $\left(\widehat{\mathscr{B}}_{t}\right)_{t \in \mathbb{R}_{+}}$defined in Lemma 12 is the augmentated filtration generated by the coordinate processes on the canonical probability space $\left(\mathbb{R}^{d} \times C\left(\mathbb{R}_{+}, \mathbb{R}^{r}\right) \times M\left(\mathbb{R}_{+} \times\right.\right.$ $\left.U), \mathscr{B}\left(\mathbb{R}^{d}\right) \otimes \mathscr{C}\left(\mathbb{R}_{+}, \mathbb{R}^{r}\right) \otimes \mathscr{M}\left(\mathbb{R}_{+} \times U\right), n \times P_{\mathrm{W}, r} \times P_{U, m}\right)$. This is the counterpart of the augmentated filtration $\left(\mathscr{F}_{t}^{\xi, W, p}\right)_{t \in \mathbb{R}_{+}}$.

The next lemma is a generalization of Corollary 1 in Yamada and Watanabe [1] (see also Problem 5.3.22 in Karatzas and Shreve [21]) for stochastic differential equations with jumps.

Lemma 14. Suppose that pathwise uniqueness holds for the $\operatorname{SDE}(1)$. If $\left(\Omega^{(i)}, \mathscr{F}^{(i)},\left(\mathscr{F}_{t}^{(i)}\right)_{t \in \mathbb{R}_{+}}, \mathbb{P}^{(i)}, \mathbf{W}^{(i)}, p^{(i)}, \mathbf{X}^{(i)}\right), i \in\{1$, $2\}$, are two weak solutions of the $S D E(1)$ with the same initial distribution $n$ on $\left(\mathbb{R}^{d}, \mathscr{B}\left(\mathbb{R}^{d}\right)\right)$, then there exists a function $k: \mathbb{R}^{d} \times C\left(\mathbb{R}_{+}, \mathbb{R}^{r}\right) \times M\left(\mathbb{R}_{+} \times U\right) \rightarrow D\left(\mathbb{R}_{+}, \mathbb{R}^{d}\right)$ such that

$$
Q_{i}(\mathbf{x}, w, \pi,\{k(\mathbf{x}, w, \pi)\})=1, \quad i \in\{1,2\}
$$

holds for $n \times P_{\mathbf{W}, r} \times P_{U, m}$-almost every $(\mathbf{x}, w, \pi) \in \mathbb{R}^{d} \times C\left(\mathbb{R}_{+}\right.$, $\left.\mathbb{R}^{r}\right) \times M\left(\mathbb{R}_{+} \times U\right)$, where $Q_{i}, i \in\{1,2\}$, is given in (33). This function $k$ is $\mathscr{B}\left(\mathbb{R}^{d}\right) \otimes \mathscr{C}\left(\mathbb{R}_{+}, \mathbb{R}^{r}\right) \otimes \mathscr{M}\left(\mathbb{R}_{+} \times U\right) / \mathscr{D}\left(\mathbb{R}_{+}, \mathbb{R}^{d}\right)$ measurable, $\widehat{\mathscr{B}}_{t} / \mathscr{D}_{t}\left(\mathbb{R}_{+}, \mathbb{R}^{d}\right)$-measurable for every fixed $t \in$ $\mathbb{R}_{+}$, and

$$
\mathbb{P}^{(i)}\left(k\left(\mathbf{X}_{0}^{(i)}, \mathbf{W}^{(i)}, p^{(i)}\right)=\mathbf{Y}^{(i)}\right)=1, \quad i \in\{1,2\} .
$$

Proof. Fix $(\mathbf{x}, w, \pi) \in \mathbb{R}^{d} \times C\left(\mathbb{R}_{+}, \mathbb{R}^{r}\right) \times M\left(\mathbb{R}_{+} \times U\right)$ and define the measure $Q_{1,2}\left(\mathbf{x}, w, \pi, \mathrm{d} y^{(1)}, \mathrm{d} y^{(2)}\right):=Q_{1}(\mathbf{x}, w, \pi$, $\left.\mathrm{d} y^{(1)}\right) Q_{2}\left(\mathbf{x}, w, \pi, \mathrm{d} y^{(2)}\right)$ on the space $S:=D\left(\mathbb{R}_{+}, \mathbb{R}^{d}\right) \times D\left(\mathbb{R}_{+}\right.$, $\left.\mathbb{R}^{d}\right)$ equipped with the $\sigma$-algebra $\mathcal{S}:=\mathscr{D}\left(\mathbb{R}_{+}, \mathbb{R}^{d}\right) \otimes \mathscr{D}\left(\mathbb{R}_{+}\right.$, $\left.\mathbb{R}^{d}\right)$. By (34) and Fubini theorem,

$$
\mathbb{P}_{1,2}[G \times B]=\int_{G} Q_{1,2}(\mathbf{x}, w, \pi, B) n(\mathrm{~d} \mathbf{x}) P_{\mathrm{W}, r}(\mathrm{~d} w) P_{U, m}(\mathrm{~d} \pi)
$$

for all $G \in \mathscr{B}\left(\mathbb{R}^{d}\right) \otimes \mathscr{C}\left(\mathbb{R}_{+}, \mathbb{R}^{r}\right) \otimes \mathscr{M}\left(\mathbb{R}_{+} \times U\right)$ and $B \in \mathcal{S}$. With the choice $G=\mathbb{R}^{d} \times C\left(\mathbb{R}_{+}, \mathbb{R}^{r}\right) \times M\left(\mathbb{R}_{+} \times U\right)$ and $B=$ $\left\{\left(y^{(1)}, y^{(2)}\right) \in S: y^{(1)}=y^{(2)}\right\}$, using that pathwise uniqueness holds for the SDE (1), relation (51) yields $\mathbb{P}_{1,2}[G \times B]=1$. Since $Q_{1,2}(\mathbf{x}, w, \pi, B) \leqslant 1$ for all $(\mathbf{x}, w, \pi) \in \mathbb{R}^{d} \times C\left(\mathbb{R}_{+}, \mathbb{R}^{r}\right) \times$ $M\left(\mathbb{R}_{+} \times U\right)$, (79) yields the existence of a set $N \in \mathscr{B}\left(\mathbb{R}^{d}\right) \otimes$ $\mathscr{C}\left(\mathbb{R}_{+}, \mathbb{R}^{r}\right) \otimes \mathscr{M}\left(\mathbb{R}_{+} \times U\right)$ with $\left(n \times P_{\mathrm{W}, r} \times P_{U, m}\right)(N)=0$ such that

$$
Q_{1,2}\left(\mathbf{x}, w, \pi,\left\{\left(y^{(1)}, y^{(2)}\right) \in S: y^{(1)}=y^{(2)}\right\}\right)=1,
$$

$$
(\mathbf{x}, w, \pi) \notin N .
$$

Again, by Fubini theorem,

$$
\begin{aligned}
1 & =Q_{1,2}\left(\mathbf{x}, w, \pi,\left\{\left(y^{(1)}, y^{(2)}\right) \in S: y^{(1)}=y^{(2)}\right\}\right) \\
& =\int_{D\left(\mathbb{R}_{+}, \mathbb{R}^{d}\right)} Q_{1}(\mathbf{x}, w, \pi,\{y\}) Q_{2}(\mathbf{x}, w, \pi, \mathrm{d} y),
\end{aligned}
$$

$$
(\mathbf{x}, w, \pi) \notin N,
$$


which can occur only if for some $y_{0} \in D\left(\mathbb{R}_{+}, \mathbb{R}^{d}\right)$, call it $\widetilde{k}(\mathbf{x}, w, \pi)$, we have

$$
Q_{i}(\mathbf{x}, w, \pi,\{\widetilde{k}(\mathbf{x}, w, \pi)\})=1, \quad i \in\{1,2\}, \quad(\mathbf{x}, w, \pi) \notin N .
$$

Indeed, since for all $(\mathbf{x}, w, \pi, y) \in \mathbb{R}^{d} \times C\left(\mathbb{R}_{+}, \mathbb{R}^{r}\right) \times M\left(\mathbb{R}_{+} \times\right.$ $U) \times D\left(\mathbb{R}_{+}, \mathbb{R}^{d}\right), Q_{1}(\mathbf{x}, w, \pi,\{y\}) \in[0,1]$, we have

$$
Q_{2}\left(\mathbf{x}, w, \pi,\left\{y \in D\left(\mathbb{R}_{+}, \mathbb{R}^{d}\right): Q_{1}(\mathbf{x}, w, \pi,\{y\})=1\right\}\right)=1,
$$$$
(\mathbf{x}, w, \pi) \notin N .
$$

Since for all $(\mathbf{x}, w, \pi) \in \mathbb{R}^{d} \times C\left(\mathbb{R}_{+}, \mathbb{R}^{r}\right) \times M\left(\mathbb{R}_{+} \times U\right)$, by (R1), the set function $\mathscr{D}\left(\mathbb{R}_{+}, \mathbb{R}^{d}\right) \ni F \mapsto Q_{i}(\mathbf{x}, w, \pi, F)$ is a probability measure on $\left(D\left(\mathbb{R}_{+}, \mathbb{R}^{d}\right), \mathscr{D}\left(\mathbb{R}_{+}, \mathbb{R}^{d}\right)\right), i \in\{1,2\}$, we get the unique existence of $\widetilde{k}(\mathbf{x}, w, \pi)$ for all $(\mathbf{x}, w, \pi) \notin N$ satisfying (82). Then we have (77) for $\widetilde{k}$.

For $(\mathbf{x}, w, \pi) \notin N$ and any $B \in \mathscr{D}\left(\mathbb{R}_{+}, \mathbb{R}^{d}\right)$, we have $\widetilde{k}(\mathbf{x}, w, \pi) \in B$ if and only if $Q_{i}(\mathbf{x}, w, \pi, B)=1, i \in\{1,2\}$.

The aim of the following discussion is to show the $\widehat{\mathscr{B}}_{t} / \mathscr{D}_{t}\left(\mathbb{R}_{+}, \mathbb{R}^{d}\right)$-measurability of $\tilde{k}$ for all $t \in \mathbb{R}_{+}$. For all $t \in \mathbb{R}_{+}$and $B \in \mathscr{D}_{t}\left(\mathbb{R}_{+}, \mathbb{R}^{d}\right)$, we have

$$
\begin{gathered}
\widetilde{k}^{-1}(B)=\left\{(\mathbf{x}, w, \pi) \in \mathbb{R}^{d} \times C\left(\mathbb{R}_{+}, \mathbb{R}^{r}\right) \times M\left(\mathbb{R}_{+} \times U\right):\right. \\
\tilde{k}(\mathbf{x}, w, \pi) \in B\}=: A_{1} \cup A_{2},
\end{gathered}
$$

where

$$
\begin{aligned}
A_{1}:=\left\{(\mathbf{x}, w, \pi) \in \mathbb{R}^{d} \times C\left(\mathbb{R}_{+}, \mathbb{R}^{r}\right) \times M\left(\mathbb{R}_{+} \times U\right):\right. \\
\tilde{k}(\mathbf{x}, w, \pi) \in B,(\mathbf{x}, w, \pi) \in N\}, \\
A_{2}:=\left\{(\mathbf{x}, w, \pi) \in \mathbb{R}^{d} \times C\left(\mathbb{R}_{+}, \mathbb{R}^{r}\right) \times M\left(\mathbb{R}_{+} \times U\right):\right. \\
\tilde{k}(\mathbf{x}, w, \pi) \in B,(\mathbf{x}, w, \pi) \notin N\} \\
=\left\{(\mathbf{x}, w, \pi) \in \mathbb{R}^{d} \times C\left(\mathbb{R}_{+}, \mathbb{R}^{r}\right) \times M\left(\mathbb{R}_{+} \times U\right):\right. \\
\quad(\mathbf{x}, w, \pi) \notin N\} \cap Q_{i}(\cdot, \cdot, \cdot B)^{-1}(\{1\})
\end{aligned}
$$

for $i \in\{1,2\}$. Lemma 12 implies $Q_{i}(\cdot, \cdot, \cdot, B)^{-1}(\{1\}) \in \widehat{\mathscr{B}}_{t}, i \in$ $\{1,2\}$. Moreover, $N \in \widehat{\mathscr{B}}_{t}$ (due to the definition of $\widehat{\mathscr{B}}_{t}$, for more details, see the proof of Lemma 12); hence $A_{2} \in \widehat{\mathscr{B}}_{t}$. Using that $A_{1} \subset N,\left(n \times P_{\mathrm{W}, r} \times P_{U, m}\right)(N)=0$ and the definition of the augmented $\sigma$-algebra $\widehat{\mathscr{B}}_{t}$ (see Lemma 12), we obtain $A_{1} \in \widehat{\mathscr{B}}_{t}$. Hence $\widetilde{k}^{-1}(B)=A_{1} \cup A_{2} \in \widehat{\mathscr{B}}_{t}$, as desired.

The aim of the following discussion is to show that $\widetilde{k}$ is

$$
\begin{aligned}
& \left({\overline{\mathscr{B}\left(\mathbb{R}^{d}\right) \otimes \mathscr{C}\left(\mathbb{R}_{+}, \mathbb{R}^{r}\right) \otimes \mathscr{M}\left(\mathbb{R}_{+} \times U\right)}}^{n \times P_{\mathrm{W}, r} \times P_{U, m}},\right. \\
& \left.\mathscr{D}\left(\mathbb{R}_{+}, \mathbb{R}^{d}\right)\right) \text {-measurable, }
\end{aligned}
$$

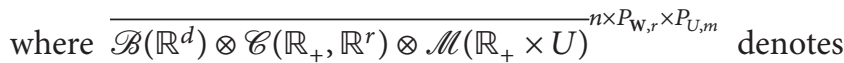
the completion of $\mathscr{B}\left(\mathbb{R}^{d}\right) \otimes \mathscr{C}\left(\mathbb{R}_{+}, \mathbb{R}^{r}\right) \otimes \mathscr{M}\left(\mathbb{R}_{+} \times U\right)$ with respect to the measure $n \times P_{\mathbf{W}, r} \times P_{U, m}$. For all $B \in \mathscr{D}\left(\mathbb{R}_{+}, \mathbb{R}^{d}\right)$, we have $\widetilde{k}^{-1}(B)=A_{1} \cup A_{2}$, where $A_{1}$ and $A_{2}$ are defined in (85). Property (R2) implies $Q_{i}(\cdot, \cdot, \cdot, B)^{-1}(\{1\}) \in \mathscr{B}\left(\mathbb{R}^{d}\right) \otimes$ $\mathscr{C}\left(\mathbb{R}_{+}, \mathbb{R}^{r}\right) \otimes \mathscr{M}\left(\mathbb{R}_{+} \times U\right), i \in\{1,2\}$. Moreover, by definition of completion (see, e.g., Definition 2.7.2 in Karatzas and Shreve [21]),

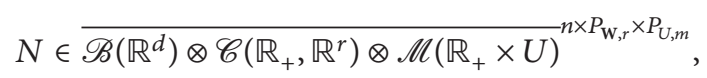

hence

$$
A_{2} \in{\overline{\mathscr{B}\left(\mathbb{R}^{d}\right) \otimes \mathscr{C}\left(\mathbb{R}_{+}, \mathbb{R}^{r}\right) \otimes \mathscr{M}\left(\mathbb{R}_{+} \times U\right)}}^{n \times P_{\mathrm{W}, r} \times P_{U, m}} .
$$

Using that $A_{1} \subset N,\left(n \times P_{\mathrm{W}, r} \times P_{U, m}\right)(N)=0$, by definition of completion, we obtain

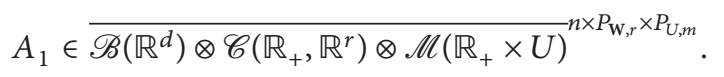

Hence

$$
\begin{aligned}
& \widetilde{k}^{-1}(B)=A_{1} \cup A_{2} \\
& \in \overline{\mathscr{B}\left(\mathbb{R}^{d}\right) \otimes \mathscr{C}\left(\mathbb{R}_{+}, \mathbb{R}^{r}\right) \otimes \mathscr{M}\left(\mathbb{R}_{+} \times U\right)}{ }^{n \times P_{\mathrm{W},} \times P_{U, m}},
\end{aligned}
$$

as desired.

Next we check (78) for $\widetilde{k}$. For $i \in\{1,2\}$, by (45), (34), (R1), and (82),

$$
\begin{array}{r}
\mathbb{P}^{(i)}\left(\widetilde{k}\left(\mathbf{X}_{0}^{(i)}, \mathbf{W}^{(i)}, p^{(i)}\right)=\mathbf{Y}^{(i)}\right) \\
=\mathbb{P}_{1,2}\left(\omega=\left(\mathbf{x}, w, \pi, y^{(1)}, y^{(2)}\right) \in \Omega: \widetilde{k}(\mathbf{x}, w, \pi)=y^{(i)}\right) \\
=\int_{\mathbb{R}^{d} \times C\left(\mathbb{R}_{+}, \mathbb{R}^{r}\right) \times M\left(\mathbb{R}_{+} \times U\right)} Q_{i}(\mathbf{x}, w, \pi,\{\widetilde{k}(\mathbf{x}, w, \pi)\}) \\
n(\mathrm{~d} \mathbf{x}) P_{\mathbf{W}, r}(\mathrm{~d} w) P_{U, m}(\mathrm{~d} \pi)=1,
\end{array}
$$

as desired.

It remains to check that one can choose a version of $\widetilde{k}$ which is $\mathscr{B}\left(\mathbb{R}^{d}\right) \otimes \mathscr{C}\left(\mathbb{R}_{+}, \mathbb{R}^{r}\right) \otimes \mathscr{M}\left(\mathbb{R}_{+} \times U\right) / \mathscr{D}\left(\mathbb{R}_{+}, \mathbb{R}^{d}\right)$ measurable, $\widehat{\mathscr{B}}_{t} / \mathscr{D}_{t}\left(\mathbb{R}_{+}, \mathbb{R}^{d}\right)$-measurable for every fixed $t \in$ $\mathbb{R}_{+}$, and (77) and (78) remain hold for $k$. Since $\widetilde{k}$ is

$$
\begin{aligned}
& \left({\overline{\mathscr{B}}\left(\mathbb{R}^{d}\right) \otimes \mathscr{C}\left(\mathbb{R}_{+}, \mathbb{R}^{r}\right) \otimes \mathscr{M}\left(\mathbb{R}_{+} \times U\right)}^{n \times P_{\mathrm{W}, r} \times P_{U, m},},\right. \\
& \left.\mathscr{D}\left(\mathbb{R}_{+}, \mathbb{R}^{d}\right)\right) \text {-measurable, }
\end{aligned}
$$

there exists a function $k: \mathbb{R}^{d} \times C\left(\mathbb{R}_{+}, \mathbb{R}^{r}\right) \times M\left(\mathbb{R}_{+} \times U\right) \rightarrow$ $D\left(\mathbb{R}_{+}, \mathbb{R}^{d}\right)$ which is $\mathscr{B}\left(\mathbb{R}^{d}\right) \otimes \mathscr{C}\left(\mathbb{R}_{+}, \mathbb{R}^{r}\right) \otimes \mathscr{M}\left(\mathbb{R}_{+} \times U\right) / \mathscr{D}\left(\mathbb{R}_{+}\right.$, $\mathbb{R}^{d}$ )-measurable and

$$
\begin{aligned}
\left(n \times P_{\mathbf{W}, r} \times P_{U, m}\right)(\{ & (\mathbf{x}, w, \pi) \in \mathbb{R}^{d} \times C\left(\mathbb{R}_{+}, \mathbb{R}^{r}\right) \\
& \times M\left(\mathbb{R}_{+} \times U\right): \\
& \widetilde{k}(\mathbf{x}, w, \pi) \neq k(\mathbf{x}, w, \pi)\})=0 ;
\end{aligned}
$$


see, for example, Cohn [22, Proposition 2.2.5]. First we check that $k$ is $\widehat{\mathscr{B}}_{t} / \mathscr{D}_{t}\left(\mathbb{R}_{+}, \mathbb{R}^{d}\right)$-measurable for every fixed $t \in \mathbb{R}_{+}$. For all $t \in \mathbb{R}_{+}$and $B \in \mathscr{D}_{t}\left(\mathbb{R}_{+}, \mathbb{R}^{d}\right)$, we have

$$
\begin{aligned}
k^{-1}(B) & =\left(k^{-1}(B) \cap\{\widetilde{k}=k\}\right) \cup\left(k^{-1}(B) \cap\{\widetilde{k} \neq k\}\right) \\
& =\left(\widetilde{k}^{-1}(B) \cap\{\widetilde{k}=k\}\right) \cup\left(k^{-1}(B) \cap\{\widetilde{k} \neq k\}\right),
\end{aligned}
$$

where $\widetilde{k}^{-1}(B) \in \widehat{\mathscr{B}}_{t}$ (since $\tilde{k}$ is $\widehat{\mathscr{B}}_{t} / \mathscr{D}_{t}\left(\mathbb{R}_{+}, \mathbb{R}^{d}\right)$-measurable), $\{\widetilde{k} \neq k\} \in \widehat{\mathscr{B}}_{t}$ (due to the definition of completion, since $(n \times$ $\left.\left.P_{\mathrm{W}, r} \times P_{U, m}\right)(\widetilde{k} \neq k)=0\right),\{\tilde{k}=k\} \in \widehat{\mathscr{B}}_{t}$ (since $\widehat{\mathscr{B}}_{t}$ is a $\sigma-$ algebra), and $k^{-1}(B) \cap\{\widetilde{k} \neq k\} \in \widehat{\mathscr{B}}_{t}$ (due to the definition of completion, since $\left.k^{-1}(B) \cap\{\widetilde{k} \neq k\} \subset\{\widetilde{k} \neq k\}\right)$. Hence $k^{-1}(B) \in$ $\widehat{\mathscr{B}}_{t}$.

Next we check (77) for $k$. Using that (77) holds for $\widetilde{k}$ and $\left(n \times P_{\mathrm{W}, r} \times P_{U, m}\right)(\tilde{k} \neq k)=0$, we have

$$
\begin{aligned}
(n \times & \left.P_{\mathbf{W}, r} \times P_{U, m}\right)\left(H_{1} \cup H_{2}\right) \\
= & \left(n \times P_{\mathbf{W}, r} \times P_{U, m}\right)\left(\left(H_{1} \cup H_{2}\right) \cap\{k=\widetilde{k}\}\right) \\
& +\left(n \times P_{\mathbf{W}, r} \times P_{U, m}\right)\left(\left(H_{1} \cup H_{2}\right) \cap\{k \neq \tilde{k}\}\right) \\
\leqslant & \left(n \times P_{\mathbf{W}, r} \times P_{U, m}\right)\left(\widetilde{H}_{1} \cup \widetilde{H}_{2}\right) \\
& +\left(n \times P_{\mathbf{W}, r} \times P_{U, m}\right)(k \neq \tilde{k})=0+0=0,
\end{aligned}
$$

where

$$
\begin{gathered}
\widetilde{H}_{i}:=\left\{(\mathbf{x}, w, \pi) \in \mathbb{R}^{d} \times C\left(\mathbb{R}_{+}, \mathbb{R}^{r}\right) \times M\left(\mathbb{R}_{+} \times U\right):\right. \\
\left.Q_{i}(\mathbf{x}, w, \pi,\{\widetilde{k}(\mathbf{x}, w, \pi)\}) \neq 1\right\}, \\
H_{i}:=\left\{(\mathbf{x}, w, \pi) \in \mathbb{R}^{d} \times C\left(\mathbb{R}_{+}, \mathbb{R}^{r}\right) \times M\left(\mathbb{R}_{+} \times U\right):\right. \\
\left.Q_{i}(\mathbf{x}, w, \pi,\{k(\mathbf{x}, w, \pi)\}) \neq 1\right\}
\end{gathered}
$$

for $i \in\{1,2\}$. This implies (77) for $k$.

Finally, we check (78) for $k$. First observe that $\mathbb{P}_{1,2}(\tilde{k}=$ $k)=1$, since, by (79),

$$
\begin{aligned}
& \mathbb{P}_{1,2}(\widetilde{k}=k) \\
& =1-\mathbb{P}_{1,2}(\widetilde{k} \neq k) \\
& =1-\int_{\{\widetilde{k} \neq k\}} Q_{1,2}\left(\mathbf{x}, w, \pi, D\left(\mathbb{R}_{+}, \mathbb{R}^{d}\right), D\left(\mathbb{R}_{+}, \mathbb{R}^{d}\right)\right) \\
& n(\mathrm{~d} \mathbf{x}) P_{\mathbf{W}, r}(\mathrm{~d} w) P_{U, m}(\mathrm{~d} \pi) \\
& =1-\int_{\{\tilde{k} \neq k\}} Q_{1}\left(\mathbf{x}, w, \pi, D\left(\mathbb{R}_{+}, \mathbb{R}^{d}\right)\right) \\
& \quad \times Q_{2}\left(\mathbf{x}, w, \pi, D\left(\mathbb{R}_{+}, \mathbb{R}^{d}\right)\right) \\
& n(\mathrm{~d} \mathbf{x}) P_{\mathbf{W}, r}(\mathrm{~d} w) P_{U, m}(\mathrm{~d} \pi) \\
& =1-\left(n \times P_{\mathbf{W}, r} \times P_{U, m}\right)(\widetilde{k} \neq k)=1-0=1,
\end{aligned}
$$

where we used (R1) as well. Then, by (45) and (34), for $i \in$ $\{1,2\}$, we obtain

$$
\begin{aligned}
& \mathbb{P}^{(i)}\left(k\left(\mathbf{X}_{0}^{(i)}, \mathbf{W}^{(i)}, p^{(i)}\right)=\mathbf{Y}^{(i)}\right) \\
& =\mathbb{P}_{1,2}\left(\omega=\left(\mathbf{x}, w, \pi, y^{(1)}, y^{(2)}\right) \in \Omega: k(\mathbf{x}, w, \pi)=y^{(i)}\right) \\
& =\mathbb{P}_{1,2}\left(\left\{\omega=\left(\mathbf{x}, w, \pi, y^{(1)}, y^{(2)}\right) \in \Omega:\right.\right. \\
& \left.\left.\quad k(\mathbf{x}, w, \pi)=y^{(i)}\right\} \cap\{\widetilde{k}=k\}\right) \\
& =\mathbb{P}_{1,2}\left(\left\{\omega=\left(\mathbf{x}, w, \pi, y^{(1)}, y^{(2)}\right) \in \Omega:\right.\right. \\
& \left.\left.\tilde{k}(\mathbf{x}, w, \pi)=y^{(i)}\right\} \cap\{\widetilde{k}=k\}\right) \\
& =\mathbb{P}_{1,2}\left(\omega=\left(\mathbf{x}, w, \pi, y^{(1)}, y^{(2)}\right) \in \Omega: \tilde{k}(\mathbf{x}, w, \pi)=y^{(i)}\right) \\
& =\mathbb{P}^{(i)}\left(\widetilde{k}\left(\mathbf{X}_{0}^{(i)}, \mathbf{W}^{(i)}, p^{(i)}\right)=\mathbf{Y}^{(i)}\right)=1,
\end{aligned}
$$

where, for the last equality, we applied that (78) holds for $\widetilde{k}$.

Remark 15. Note that the function $k$ in Lemma 14 and the $n \times$ $P_{\mathrm{W}, r} \times P_{U, m}$-null set on which (77) does not hold depend on the two weak solutions in question.

Applying Lemma 14 for weak solutions $\left(\Omega^{(i)}, \mathscr{F}^{(i)}\right.$, $\left.\left(\mathscr{F}_{t}^{(i)}\right)_{t \in \mathbb{R}_{+}}, \mathbb{P}^{(i)}, \mathbf{W}^{(i)}, p^{(i)}, \mathbf{X}^{(i)}\right)=\left(\Omega, \mathscr{F},\left(\mathscr{F}_{t}\right)_{t \in \mathbb{R}_{+}}, \mathbb{P}, \mathbf{W}, p, \mathbf{X}\right)$, $i \in\{1,2\}$, of the SDE (1) with the same initial distribution $n$ on $\left(\mathbb{R}^{d}, \mathscr{B}\left(\mathbb{R}^{d}\right)\right)$, we obtain the following corollary.

Corollary 16. If pathwise uniqueness holds for the SDE (1) and $\left(\Omega, \mathscr{F},\left(\mathscr{F}_{t}\right)_{t \in \mathbb{R}_{+}}, \mathbb{P}, \mathbf{W}, p, \mathbf{X}\right)$ is a weak solution of the SDE (1) with initial distribution $n$ on $\left(\mathbb{R}^{d}, \mathscr{B}\left(\mathbb{R}^{d}\right)\right)$, then there exists a function $k: \mathbb{R}^{d} \times C\left(\mathbb{R}_{+}, \mathbb{R}^{r}\right) \times M\left(\mathbb{R}_{+} \times U\right) \rightarrow D\left(\mathbb{R}_{+}, \mathbb{R}^{d}\right)$ such that $Q(\mathbf{x}, w, \pi,\{k(\mathbf{x}, w, \pi)\})=1$ holds for $n \times P_{\mathbf{W}, r} \times P_{U, m}$-almost every $(\mathbf{x}, w, \pi) \in \mathbb{R}^{d} \times C\left(\mathbb{R}_{+}, \mathbb{R}^{r}\right) \times M\left(\mathbb{R}_{+} \times U\right)$, where $Q$ is given in (29). This function $k$ is $\mathscr{B}\left(\mathbb{R}^{d}\right) \otimes \mathscr{C}\left(\mathbb{R}_{+}, \mathbb{R}^{r}\right) \otimes \mathscr{M}\left(\mathbb{R}_{+} \times\right.$ $U) / \mathscr{D}\left(\mathbb{R}_{+}, \mathbb{R}^{d}\right)$-measurable, $\widehat{\mathscr{B}}_{t} / \mathscr{D}_{t}\left(\mathbb{R}_{+}, \mathbb{R}^{d}\right)$-measurable for every fixed $t \in \mathbb{R}_{+}$, and $\mathbb{P}\left(k\left(\mathbf{X}_{0}, \mathbf{W}, p\right)=\mathbf{Y}\right)=1$.

Next we give the precise formulation of Theorem 2.

Theorem 17. Suppose that pathwise uniqueness holds for the $\operatorname{SDE}(1)$ and there exists a weak solution $\left(\Omega^{\prime}, \mathscr{F}^{\prime},\left(\mathscr{F}_{t}^{\prime}\right)_{t \in \mathbb{R}_{+}}, \mathbb{P}^{\prime}\right.$, $\left.\mathbf{W}^{\prime}, p^{\prime}, \mathbf{X}^{\prime}\right)$ of the SDE (1) with initial distribution $n^{\prime}$. Then there exists a function $h^{\prime}: \mathbb{R}^{d} \times C\left(\mathbb{R}_{+}, \mathbb{R}^{r}\right) \times M\left(\mathbb{R}_{+} \times\right.$ $U) \rightarrow D\left(\mathbb{R}_{+}, \mathbb{R}^{d}\right)$ which is $\mathscr{B}\left(\mathbb{R}^{d}\right) \otimes \mathscr{C}\left(\mathbb{R}_{+}, \mathbb{R}^{r}\right) \otimes \mathscr{M}\left(\mathbb{R}_{+} \times\right.$ $U) / \mathscr{D}\left(\mathbb{R}_{+}, \mathbb{R}^{d}\right)$-measurable, $\widehat{\mathscr{B}}_{t} / \mathscr{D}_{t}\left(\mathbb{R}_{+}, \mathbb{R}^{d}\right)$-measurable for every fixed $t \in \mathbb{R}_{+}$, and

$$
\mathbf{X}^{\prime}=h^{\prime}\left(\mathbf{X}_{0}^{\prime}, \mathbf{W}^{\prime}, p^{\prime}\right) \quad \mathbb{P}^{\prime} \text {-almost surely. }
$$

Moreover, if objects (E1)-(E4) are given such that the distribution of $\xi$ is $n^{\prime}$, then the process

$$
\mathbf{X}:=h^{\prime}(\boldsymbol{\xi}, \mathbf{W}, p)
$$

is a strong solution of the SDE (1) with initial value $\xi$. 
Proof. Let $h^{\prime}(\mathbf{x}, w, \pi):=\mathbf{x}+k^{\prime}(\mathbf{x}, w, \pi)$ for $\mathbf{x} \in \mathbb{R}^{d}, w \in$ $C\left(\mathbb{R}_{+}, \mathbb{R}^{r}\right), \pi \in M\left(\mathbb{R}_{+} \times U\right)$, where $k^{\prime}$ is as in Corollary 16 . By Corollary 16, for the function $h^{\prime}$, the desired measurability properties hold. Using Corollary 16 and $\mathbf{X}^{\prime}=\mathbf{X}_{0}^{\prime}+\mathbf{Y}^{\prime}$, we have

$$
\begin{aligned}
\mathbb{P}^{\prime} & \left(\mathbf{X}^{\prime}=h^{\prime}\left(\mathbf{X}_{0}^{\prime}, \mathbf{W}^{\prime}, p^{\prime}\right)\right) \\
& =\mathbb{P}^{\prime}\left(\mathbf{X}_{0}^{\prime}+\mathbf{Y}^{\prime}=\mathbf{X}_{0}^{\prime}+k^{\prime}\left(\mathbf{X}_{0}^{\prime}, \mathbf{W}^{\prime}, p^{\prime}\right)\right) \\
& =\mathbb{P}^{\prime}\left(\mathbf{Y}^{\prime}=k^{\prime}\left(\mathbf{X}_{0}^{\prime}, \mathbf{W}^{\prime}, p^{\prime}\right)\right)=1,
\end{aligned}
$$

implying (99).

Note that, for $\boldsymbol{\xi}, \mathbf{W}$, and $p$ as described in (E1)-(E4), the triplets $\left(\mathbf{X}_{0}^{\prime}, \mathbf{W}^{\prime}, p^{\prime}\right)$ and $(\boldsymbol{\xi}, \mathbf{W}, p)$ induce the same probability measure $n^{\prime} \times P_{\mathbf{W}, r} \times P_{U, m}$ on the measurable space

$$
\begin{aligned}
\left(\mathbb{R}^{d} \times C\left(\mathbb{R}_{+}, \mathbb{R}^{r}\right) \times M\left(\mathbb{R}_{+} \times U\right),\right. \\
\left.\quad \mathscr{B}\left(\mathbb{R}^{d}\right) \otimes \mathscr{C}\left(\mathbb{R}_{+}, \mathbb{R}^{r}\right) \otimes \mathscr{M}\left(\mathbb{R}_{+} \times U\right)\right)
\end{aligned}
$$

with respect to the probability measure $\mathbb{P}^{\prime}$ and $\mathbb{P}$, respectively, where $\mathbb{P}$ denotes the probability measure appears in (E1), since $\mathbf{X}_{0}^{\prime}, \mathbf{W}^{\prime}$, and $p^{\prime}$ are $\mathbb{P}^{\prime}$-independent and $\boldsymbol{\xi}, \mathbf{W}$, and $p$ are $\mathbb{P}$-independent; see Remarks 6 and 10.

Observe also that the mappings

$$
\begin{aligned}
& \Omega^{\prime} \ni \omega^{\prime} \longmapsto\left(\mathbf{X}_{0}^{\prime}\left(\omega^{\prime}\right),\left(\mathbf{W}_{t}^{\prime}\left(\omega^{\prime}\right)\right)_{t \in \mathbb{R}_{+}}, N_{p^{\prime}\left(\omega^{\prime}\right)}\right) \\
& \in \mathbb{R}^{d} \times C\left(\mathbb{R}_{+}, \mathbb{R}^{r}\right) \times M\left(\mathbb{R}_{+} \times U\right), \\
& \Omega \ni \omega \longmapsto\left(\xi(\omega),\left(\mathbf{W}_{t}(\omega)\right)_{t \in \mathbb{R}_{+}}, N_{p(\omega)}\right) \\
& \quad \in \mathbb{R}^{d} \times C\left(\mathbb{R}_{+}, \mathbb{R}^{r}\right) \times M\left(\mathbb{R}_{+} \times U\right)
\end{aligned}
$$

are $\mathscr{F}^{\prime} / \mathscr{B}\left(\mathbb{R}^{d}\right) \otimes \mathscr{C}\left(\mathbb{R}_{+}, \mathbb{R}^{r}\right) \otimes \mathscr{M}\left(\mathbb{R}_{+} \times U\right)$-measurable and

$$
\begin{aligned}
& \left(\sigma\left(\xi, \mathbf{W}_{s}, s \in \mathbb{R}_{+}, p(s), s \in \mathbb{R}_{++} \cap D(p)\right)\right. \\
& \left.\quad \mathscr{B}\left(\mathbb{R}^{d}\right) \otimes \mathscr{C}\left(\mathbb{R}_{+}, \mathbb{R}^{r}\right) \otimes \mathscr{M}\left(\mathbb{R}_{+} \times U\right)\right) \text {-measurable, }
\end{aligned}
$$

respectively. Further, they are $\mathscr{F}_{t}^{\prime} / \mathscr{B}\left(\mathbb{R}^{d}\right) \otimes \mathscr{C}_{t}\left(\mathbb{R}_{+}, \mathbb{R}^{r}\right) \otimes$ $\mathscr{M}_{t}\left(\mathbb{R}_{+} \times U\right)$-measurable and

$$
\begin{aligned}
& \left(\sigma\left(\xi, \mathbf{W}_{s}, s \in[0, t], p(s), s \in(0, t] \cap D(p)\right),\right. \\
& \left.\quad \mathscr{B}\left(\mathbb{R}^{d}\right) \otimes \mathscr{C}_{t}\left(\mathbb{R}_{+}, \mathbb{R}^{r}\right) \otimes \mathscr{M}_{t}\left(\mathbb{R}_{+} \times U\right)\right) \text {-measurable }
\end{aligned}
$$

for all $t \in \mathbb{R}_{+}$, respectively. Indeed, since $\mathbf{X}_{0}^{\prime}$ and $\boldsymbol{\xi}$ are $\mathscr{F}^{\prime} /$ $\mathscr{B}\left(\mathbb{R}^{d}\right)$-measurable and $\sigma(\xi) / \mathscr{B}\left(\mathbb{R}^{d}\right)$-measurable, respectively, by (11) and (15), it is enough to check that for all $t \in \mathbb{R}_{+}$, $n \in \mathbb{N}, A_{1} \in \mathscr{B}\left(\mathbb{R}^{n r}\right), t_{1}, \ldots, t_{n} \in[0, t], s \in[0, t], B \in \mathscr{B}(U)$, $A_{2} \in \mathscr{B}([0, \infty])$,

$$
\left\{\omega^{\prime} \in \Omega^{\prime}:\left(\mathbf{W}_{t_{1}}^{\prime}\left(\omega^{\prime}\right), \ldots, \mathbf{W}_{t_{n}}^{\prime}\left(\omega^{\prime}\right)\right) \in A_{1}\right\} \in \mathscr{F}^{\prime},
$$

$\left\{\omega \in \Omega:\left(\mathbf{W}_{t_{1}}(\omega), \ldots, \mathbf{W}_{t_{n}}(\omega)\right) \in A_{1}\right\} \in \sigma\left(\mathbf{W}_{s}, s \in \mathbb{R}_{+}\right)$,

$$
\begin{gathered}
\left\{\omega^{\prime} \in \Omega^{\prime}: N_{p^{\prime}\left(\omega^{\prime}\right)}([0, s] \times B) \in A_{2}\right\} \in \mathscr{F}^{\prime}, \\
\left\{\omega \in \Omega: N_{p(\omega)}([0, s] \times B) \in A_{2}\right\} \\
\in \sigma\left(p(s), s \in \mathbb{R}_{++} \cap D(p)\right) .
\end{gathered}
$$

These relations hold since $\mathbf{W}_{t_{i}}^{\prime}, i \in\{1, \ldots, n\}$, and $\mathbf{W}_{t_{i}}, i \in\{1$, $\ldots, n\}$, are $\mathscr{F}^{\prime} / \mathscr{B}\left(\mathbb{R}^{r}\right)$-measurable and $\sigma\left(\mathbf{W}_{s}, s \in \mathbb{R}_{+}\right) / \mathscr{B}\left(\mathbb{R}^{r}\right)$ measurable, and $p^{\prime}$ and $p$ are $\mathscr{F}^{\prime} / \mathscr{M}\left(\mathbb{R}_{+} \times U\right)$-measurable and $\sigma\left(p(s), s \in \mathbb{R}_{++} \cap D(p)\right) / \mathscr{M}\left(\mathbb{R}_{+} \times U\right)$-measurable, respectively. Similarly, one can argue that the functions in question are $\mathscr{F}_{t}^{\prime} / \mathscr{B}\left(\mathbb{R}^{d}\right) \otimes \mathscr{C}_{t}\left(\mathbb{R}_{+}, \mathbb{R}^{r}\right) \otimes \mathscr{M}_{t}\left(\mathbb{R}_{+} \times U\right)$-measurable and $\sigma\left(\boldsymbol{\xi}, \mathbf{W}_{s}, s \in[0, t], p(s), s \in(0, t] \cap D(p)\right) / \mathscr{B}\left(\mathbb{R}^{d}\right) \otimes \mathscr{C}_{t}\left(\mathbb{R}_{+}\right.$, $\left.\mathbb{R}^{r}\right) \otimes \mathscr{M}_{t}\left(\mathbb{R}_{+} \times U\right)$-measurable for all $t \in \mathbb{R}_{+}$, respectively.

Next, we check that the process $\mathbf{X}$ is adapted to the augmented filtration $\left(\mathscr{F}_{t}^{\xi, \mathbf{W}, p}\right)_{t \in \mathbb{R}_{+}}$. First, note that the process $\mathbf{X}$ is adapted to $\left(\mathscr{F}_{t}^{\xi, \mathbf{W}, p}\right)_{t \in \mathbb{R}_{+}}$if and only if $\varphi_{t}(\mathbf{X})$ is $\mathscr{F}_{t}^{\xi, \mathbf{W}, p} / \mathscr{D}_{t}\left(\mathbb{R}_{+}, \mathbb{R}^{d}\right)$-measurable for all $t \in \mathbb{R}_{+}$, where $\varphi_{t}$ is given in (9). Indeed,

$$
\begin{aligned}
&\left(\mathbf{X}_{t}\right)_{t \in \mathbb{R}_{+}} \text {is }\left(\mathscr{F}_{t}^{\xi, \mathbf{W}, p}\right)_{t \in \mathbb{R}_{+}} \text {-adapted } \\
& \Longleftrightarrow \sigma\left(\mathbf{X}_{t}\right) \subset \mathscr{F}_{t}^{\xi, \mathbf{W}, p} \quad \forall t \in \mathbb{R}_{+} \\
& \Longleftrightarrow \sigma\left(\mathbf{X}_{s}: s \in[0, t]\right) \subset \mathscr{F}_{t}^{\xi, \mathbf{W}, p} \quad \forall t \in \mathbb{R}_{+} \\
& \Longleftrightarrow \varphi_{t}(\mathbf{X}) \text { is }\left(\mathscr{F}_{t}^{\xi, \mathbf{W}, p}, \mathscr{D}_{t}\left(\mathbb{R}_{+}, \mathbb{R}^{d}\right)\right) \text {-measurable } \\
& \forall t \in \mathbb{R}_{+},
\end{aligned}
$$

where the last equivalence can be checked as follows. Since $\mathscr{D}_{t}\left(\mathbb{R}_{+}, \mathbb{R}^{d}\right)$ coincides with the smallest $\sigma$-algebra containing the finite-dimensional cylinder sets of the form

$$
\begin{aligned}
& \left\{y \in D\left(\mathbb{R}_{+}, \mathbb{R}^{d}\right):\left(y\left(t_{1}\right), \ldots, y\left(t_{n}\right)\right) \in A\right\}, \\
& n \in \mathbb{N}, \quad A \in \mathscr{B}\left(\mathbb{R}^{n d}\right), \quad t_{1}, \ldots, t_{n} \in[0, t],
\end{aligned}
$$

it is enough to check that $\sigma\left(\mathbf{X}_{s}: s \in[0, t]\right) \subset \mathscr{F}_{t}^{\xi, \mathbf{W}, p}$ for all $t \in \mathbb{R}_{+}$is equivalent with

$$
\left\{\omega \in \Omega:\left(\left(\varphi_{t}(\mathbf{X})\right)_{t_{1}}(\omega), \ldots,\left(\varphi_{t}(\mathbf{X})\right)_{t_{n}}(\omega)\right) \in A\right\} \in \mathscr{F}_{t}^{\xi, \mathbf{W}, p}
$$

for all $n \in \mathbb{N}, A \in \mathscr{B}\left(\mathbb{R}^{n d}\right), t_{1}, \ldots, t_{n} \in[0, t], t \in \mathbb{R}_{+}$, which readily follows from

$$
\begin{aligned}
\{\omega & \left.\in \Omega:\left(\left(\varphi_{t}(\mathbf{X})\right)_{t_{1}}(\omega), \ldots,\left(\varphi_{t}(\mathbf{X})\right)_{t_{n}}(\omega)\right) \in A\right\} \\
& =\left\{\omega \in \Omega:\left(\mathbf{X}_{t_{1}}(\omega), \ldots, \mathbf{X}_{t_{n}}(\omega)\right) \in A\right\} .
\end{aligned}
$$

Since $\varphi_{t}(\mathbf{X})=\varphi_{t} \circ h^{\prime} \circ(\boldsymbol{\xi}, \mathbf{W}, p), t \in \mathbb{R}_{+}$, the mapping $\varphi_{t}$ is $\mathscr{D}_{t}\left(\mathbb{R}_{+}, \mathbb{R}^{d}\right) / \mathscr{D}_{t}\left(\mathbb{R}_{+}, \mathbb{R}^{d}\right)$-measurable for all $t \in \mathbb{R}_{+}, h^{\prime}$ is $\widehat{\mathscr{B}}_{t} /$ $\mathscr{D}_{t}\left(\mathbb{R}_{+}, \mathbb{R}^{d}\right)$-measurable for all $t \in \mathbb{R}_{+}$, it remains to check 
that the mapping (104) is $\mathscr{F}_{t}^{\xi, \mathrm{W}, p} / \widehat{\mathscr{B}}_{t}$-measurable for all $t \epsilon$ $\mathbb{R}_{+}$. Recall that

$$
\begin{gathered}
\widehat{\mathscr{B}}_{t}=\sigma\left(\mathscr{B}\left(\mathbb{R}^{d}\right) \otimes \mathscr{C}_{t}\left(\mathbb{R}_{+}, \mathbb{R}^{r}\right) \otimes \mathscr{M}_{t}\left(\mathbb{R}_{+} \times U\right) \cup \mathcal{N}\right), \\
t \in \mathbb{R}_{+}, \\
\mathscr{F}_{t}^{\xi, \mathbf{W}, p}=\sigma\left(\sigma\left(\xi, \mathbf{W}_{s}, s \in[0, t], p(s), s \in(0, t] \cap D(p)\right)\right. \\
\left.\cup \mathcal{N}^{\xi, \mathbf{W}, p}\right), \quad t \in \mathbb{R}_{+},
\end{gathered}
$$

where

$$
\begin{gathered}
\mathcal{N}=\left\{A \subset \mathbb{R}^{d} \times C\left(\mathbb{R}_{+}, \mathbb{R}^{r}\right) \times M\left(\mathbb{R}_{+} \times U\right):\right. \\
\exists B \in \mathscr{B}\left(\mathbb{R}^{d}\right) \otimes \mathscr{C}\left(\mathbb{R}_{+}, \mathbb{R}^{r}\right) \otimes \mathscr{M}\left(\mathbb{R}_{+} \times U\right) \\
\text { with } \left.A \subset B,\left(n^{\prime} \times P_{\mathbf{W}, r} \times P_{U, m}\right)(B)=0\right\}, \\
\mathcal{N}^{\xi, \mathbf{W}, p}:=\{A \subset \Omega: \\
\exists B \in \sigma\left(\xi, \mathbf{W}_{s}, s \in \mathbb{R}_{+}, p(s), s \in \mathbb{R}_{++} \cap D(p)\right) \\
\text { with } A \subset B, \mathbb{P}(B)=0\} .
\end{gathered}
$$

Since a generator system of $\mathscr{B}\left(\mathbb{R}^{d}\right) \otimes \mathscr{C}_{t}\left(\mathbb{R}_{+}, \mathbb{R}^{r}\right) \otimes \mathscr{M}_{t}\left(\mathbb{R}_{+} \times U\right)$ together with $\mathcal{N}$ is a generator system of $\widehat{\mathscr{B}}_{t}$, and we have already checked that the mapping (104) is

$$
\begin{aligned}
& \left(\sigma\left(\xi, \mathbf{W}_{s}, s \in \mathbb{R}_{+}, p(s), s \in \mathbb{R}_{++} \cap D(p)\right),\right. \\
& \left.\mathscr{B}\left(\mathbb{R}^{d}\right) \otimes \mathscr{C}\left(\mathbb{R}_{+}, \mathbb{R}^{r}\right) \otimes \mathscr{M}\left(\mathbb{R}_{+} \times U\right)\right) \text {-measurable, }
\end{aligned}
$$

it remains to verify that $(\boldsymbol{\xi}, \mathbf{W}, p)^{-1}(A) \in \mathscr{F}_{t}^{\xi, \mathbf{W}, p}$ for all $A \in$ $\mathcal{N}$ and $t \in \mathbb{R}_{+}$. We show that $(\boldsymbol{\xi}, \mathbf{W}, p)^{-1}(A) \in \mathcal{N}^{\xi, \mathbf{W}, p}$ for all $A \in \mathcal{N}$, implying $(\boldsymbol{\xi}, \mathbf{W}, p)^{-1}(A) \in \mathscr{F}_{t}^{\xi, \mathbf{W}, p}$ for all $t \in \mathbb{R}_{+}$, as desired. If $A \in \mathcal{N}$, then there exists $B \in \mathscr{B}\left(\mathbb{R}^{d}\right) \otimes \mathscr{C}\left(\mathbb{R}_{+}, \mathbb{R}^{r}\right) \otimes$ $\mathscr{M}\left(\mathbb{R}_{+} \times U\right)$ such that $A \subset B$ and $\left(n^{\prime} \times P_{\mathbf{W}, r} \times P_{U, m}\right)(B)=0$. Hence

$$
\begin{aligned}
& (\boldsymbol{\xi}, \mathbf{W}, p)^{-1}(A) \\
& \subseteq(\boldsymbol{\xi}, \mathbf{W}, p)^{-1}(B) \\
& \quad \in \sigma\left(\xi, \mathbf{W}_{s}, s \in \mathbb{R}_{+}, p(s), s \in \mathbb{R}_{++} \cap D(p)\right), \\
& \mathbb{P}\left((\xi, \mathbf{W}, p)^{-1}(B)\right)=\mathbb{P}((\xi, \mathbf{W}, p) \in B) \\
& \quad=\left(n^{\prime} \times P_{\mathbf{W}, r} \times P_{U, m}\right)(B)=0,
\end{aligned}
$$

where, for the last but one equality, we used that the distribution of $(\xi, \mathbf{W}, p)$ under $\mathbb{P}$ is $n^{\prime} \times P_{\mathbf{W}, r} \times P_{U, m}$ (as it was explained at the beginning of the proof). By definition, this means that $(\boldsymbol{\xi}, \mathbf{W}, p)^{-1}(A) \in \mathcal{N}^{\xi, \mathbf{W}, p}$.
Next we check that $\left(\mathbf{X}_{t}\right)_{t \in \mathbb{R}_{+}}$satisfies the $\operatorname{SDE}(1) \mathbb{P}$-almost surely. Since $h^{\prime}$ is $\mathscr{B}\left(\mathbb{R}^{d}\right) \otimes \mathscr{C}\left(\mathbb{R}_{+}, \mathbb{R}^{r}\right) \otimes \mathscr{M}\left(\mathbb{R}_{+} \times U\right) / \mathscr{D}\left(\mathbb{R}_{+}\right.$, $\left.\mathbb{R}^{d}\right)$-measurable, and the triplets $\left(\mathbf{X}_{0}^{\prime}, \mathbf{W}^{\prime}, p^{\prime}\right)$ and $(\xi, \mathbf{W}, p)$ induce the same probability measure $n^{\prime} \times P_{\mathrm{W}, r} \times P_{U, m}$ on the measurable space

$$
\begin{aligned}
\left(\mathbb{R}^{d} \times C\left(\mathbb{R}_{+}, \mathbb{R}^{r}\right) \times M\left(\mathbb{R}_{+} \times U\right),\right. \\
\left.\mathscr{B}\left(\mathbb{R}^{d}\right) \otimes \mathscr{C}\left(\mathbb{R}_{+}, \mathbb{R}^{r}\right) \otimes \mathscr{M}\left(\mathbb{R}_{+} \times U\right)\right)
\end{aligned}
$$

with respect to the probability measure $\mathbb{P}^{\prime}$ and $\mathbb{P}$, respectively, the triplets $\left(\mathbf{X}^{\prime}, \mathbf{W}^{\prime}, p^{\prime}\right)$ and $(\mathbf{X}, \mathbf{W}, p)$ induce the same probability measure on the measurable space

$$
\begin{aligned}
& \left(D\left(\mathbb{R}_{+}, \mathbb{R}^{d}\right) \times C\left(\mathbb{R}_{+}, \mathbb{R}^{r}\right) \times M\left(\mathbb{R}_{+} \times U\right),\right. \\
& \left.\mathscr{D}\left(\mathbb{R}_{+}, \mathbb{R}^{d}\right) \otimes \mathscr{C}\left(\mathbb{R}_{+}, \mathbb{R}^{r}\right) \otimes \mathscr{M}\left(\mathbb{R}_{+} \times U\right)\right)
\end{aligned}
$$

with respect to the probability measure $\mathbb{P}^{\prime}$ and $\mathbb{P}$, respectively. Let us apply Lemma A.4 with the following choices:

$$
\begin{gathered}
\left(\Omega^{(1)}, \mathscr{F}^{(1)},\left(\mathscr{F}_{t}^{(1)}\right)_{t \in \mathbb{R}_{+}}, \mathbb{P}^{(1)}, \mathbf{W}^{(1)}, p^{(1)}, \mathbf{X}^{(1)}\right) \\
:=\left(\Omega^{\prime}, \mathscr{F}^{\prime},\left(\mathscr{F}_{t}^{\prime}\right)_{t \in \mathbb{R}_{+}}, \mathbb{P}^{\prime}, \mathbf{W}^{\prime}, p^{\prime}, \mathbf{X}^{\prime}\right), \\
\left(\Omega^{(2)}, \mathscr{F}^{(2)},\left(\mathscr{F}_{t}^{(2)}\right)_{t \in \mathbb{R}_{+}}, \mathbb{P}^{(2)}, \mathbf{W}^{(2)}, p^{(2)}, \mathbf{X}^{(2)}\right) \\
:=\left(\Omega, \mathscr{F},\left(\mathscr{F}_{t}^{\xi, \mathbf{W}, p}\right)_{t \in \mathbb{R}_{+}}, \mathbb{P}, \mathbf{W}, p, \mathbf{X}\right) .
\end{gathered}
$$

Since $\left(\Omega^{\prime}, \mathscr{F}^{\prime},\left(\mathscr{F}_{t}^{\prime}\right)_{t \in \mathbb{R}_{+}}, \mathbb{P}^{\prime}, \mathbf{W}^{\prime}, p^{\prime}, \mathbf{X}^{\prime}\right)$ is a weak solution of the SDE (1) with initial distribution $n^{\prime}$, the tuple $\left(\Omega^{(1)}, \mathscr{F}^{(1)}\right.$, $\left.\left(\mathscr{F}_{t}^{(1)}\right)_{t \in \mathbb{R}_{+}}, \mathbb{P}^{(1)}, \mathbf{W}^{(1)}, p^{(1)}, \mathbf{X}^{(1)}\right)$ satisfies (D1), (D2), (D3), and (D4)(b)-(e). Further, as it was explained before Definition 11, the tuple $\left(\Omega^{(2)}, \mathscr{F}^{(2)},\left(\mathscr{F}_{t}^{(2)}\right)_{t \in \mathbb{R}_{+}}, \mathbb{P}^{(2)}, \mathbf{W}^{(2)}, p^{(2)}, \mathbf{X}^{(2)}\right)$ satisfies (D1), (D2), and (D3), and we have already checked that $\mathbf{X}$ is adapted to the augmented filtration $\left(\mathscr{F}_{t}^{\xi, \mathbf{W}, p}\right)_{t \in \mathbb{R}_{+}}$. Then Lemma A.4 yields that the tuple $\left(\Omega^{(2)}, \mathscr{F}^{(2)},\left(\mathscr{F}_{t}^{(2)}\right)_{t \in \mathbb{R}_{+}}\right.$, $\left.\mathbb{P}^{(2)}, \mathbf{W}^{(2)}, p^{(2)}, \mathbf{X}^{(2)}\right)$ satisfies (D4)(b)-(d) and the distribution of

$$
\begin{gathered}
\left(\mathbf{X}_{t}^{\prime}-\mathbf{X}_{0}^{\prime}-\int_{0}^{t} b\left(s, \mathbf{X}_{s}^{\prime}\right) \mathrm{d} s-\int_{0}^{t} \sigma\left(s, \mathbf{X}_{s}^{\prime}\right) \mathrm{d} \mathbf{W}_{s}^{\prime}\right. \\
-\int_{0}^{t} \int_{U_{0}} f\left(s, \mathbf{X}_{s^{-}}^{\prime}, u\right) \widetilde{N}^{\prime}(\mathrm{d} s, \mathrm{~d} u) \\
\left.-\int_{0}^{t} \int_{U_{1}} g\left(s, \mathbf{X}_{s^{-}}^{\prime}, u\right) N^{\prime}(\mathrm{d} s, \mathrm{~d} u)\right)_{t \in \mathbb{R}_{+}}
\end{gathered}
$$


on $\left(D\left(\mathbb{R}_{+}, \mathbb{R}^{d}\right), \mathscr{D}\left(\mathbb{R}_{+}, \mathbb{R}^{d}\right)\right)$ under $\mathbb{P}^{\prime}$ is the same as the distribution of

$$
\begin{gathered}
\left(\mathbf{X}_{t}-\mathbf{X}_{0}-\int_{0}^{t} b\left(s, \mathbf{X}_{s}\right) \mathrm{d} s-\int_{0}^{t} \sigma\left(s, \mathbf{X}_{s}\right) \mathrm{d} \mathbf{W}_{s}\right. \\
-\int_{0}^{t} \int_{U_{0}} f\left(s, \mathbf{X}_{s-}, u\right) \widetilde{N}(\mathrm{~d} s, \mathrm{~d} u) \\
\left.-\int_{0}^{t} \int_{U_{1}} g\left(s, \mathbf{X}_{s-}, u\right) N(\mathrm{~d} s, \mathrm{~d} u)\right)_{t \in \mathbb{R}_{+}}
\end{gathered}
$$

on $\left(D\left(\mathbb{R}_{+}, \mathbb{R}^{d}\right), \mathscr{D}\left(\mathbb{R}_{+}, \mathbb{R}^{d}\right)\right)$ under $\mathbb{P}$, where $N^{\prime}(\mathrm{d} s, \mathrm{~d} u)$ and $N(\mathrm{~d} s, \mathrm{~d} u)$ is the counting measure of $p^{\prime}$ and $p$ on $\mathbb{R}_{+} \times U$, respectively, and $\widetilde{N}^{\prime}(\mathrm{d} s, \mathrm{~d} u):=N^{\prime}(\mathrm{d} s, \mathrm{~d} u)-\mathrm{d} s m(\mathrm{~d} u)$ and $\widetilde{N}(\mathrm{~d} s, \mathrm{~d} u):=N(\mathrm{~d} s, \mathrm{~d} u)-\mathrm{d} s m(\mathrm{~d} u)$. Using that the first process and the identically 0 process are indistinguishable (since the SDE (1) holds $\mathbb{P}^{\prime}$-a.s. for $\left.\left(\mathbf{X}_{t}^{\prime}\right)_{t \in \mathbb{R}_{+}}\right)$, we obtain that the SDE (1) holds $\mathbb{P}$-a.s. for $\left(\mathbf{X}_{t}\right)_{t \in \mathbb{R}_{+}}$as well, that is, (D4)(e) holds.

Finally, we show that $\mathbb{P}\left(\mathbf{X}_{0}=\xi\right)=1$. Since, as it was checked that the distribution of $\mathbf{X}^{\prime}$ and $\mathbf{X}$ coincide, especially, the distribution of $\mathbf{X}_{0}^{\prime}$ and $\mathbf{X}_{0}$ coincide, and consequently, the distribution of $\mathbf{X}_{0}$ and $\boldsymbol{\xi}$ coincide (both are equal to $\left.n^{\prime}\right)$. Using Corollary 16 for $\left(\Omega, \mathscr{F},\left(\mathscr{F}_{t}^{\xi, \mathbf{W}, p}\right)_{t \in \mathbb{R}_{+}}, \mathbb{P}, \mathbf{W}, p, \mathbf{X}\right)$ (which is especially a weak solution of the SDE (1) with initial distribution $n^{\prime}$ ) we get

$$
\begin{aligned}
\mathbb{P}\left(\mathbf{X}_{0}=\boldsymbol{\xi}\right) & =\mathbb{P}\left(\boldsymbol{\xi}+k^{\prime}(\boldsymbol{\xi}, \mathbf{W}, p)_{0}=\boldsymbol{\xi}\right) \\
& =\mathbb{P}\left(k^{\prime}(\boldsymbol{\xi}, \mathbf{W}, p)_{0}=\mathbf{0}\right) \\
& =\mathbb{P}\left(k^{\prime}(\boldsymbol{\xi}, \mathbf{W}, p)_{0}=\mathbf{Y}_{0}\right)=1,
\end{aligned}
$$

as desired.

Summarizing, $\left(\mathbf{X}_{t}\right)_{t \in \mathbb{R}_{+}}$is a strong solution of the SDE (1) with initial value $\xi$.

\section{Appendix}

Let $\left(\Omega, \mathscr{F},\left(\mathscr{F}_{t}\right)_{t \in \mathbb{R}_{+}}, \mathbb{P}\right)$ be a filtered probability space. First we recall the notion of $\left(\mathscr{F}_{t}\right)_{t \in \mathbb{R}_{+}}$-predictability; see, for example, Ikeda and Watanabe [20, Chapter II, Definition 3.3]. The predictable $\sigma$-algebra $\mathscr{P}$ on $\mathbb{R}_{+} \times \Omega \times U$ is given by

$$
\begin{gathered}
\mathscr{P}:=\sigma\left(h: \mathbb{R}_{+} \times \Omega \times U \longrightarrow \mathbb{R} \mid\right. \\
h(t, \cdot, \cdot) \text { is }\left(\mathscr{F}_{t} \otimes \mathscr{B}(U), \mathscr{B}(\mathbb{R})\right) \text {-measurable } \\
\forall t \in \mathbb{R}_{++}, \\
h(\cdot, \omega, u) \text { is left continuous } \forall(\omega, u) \in \Omega \times U) .
\end{gathered}
$$

A function $H: \mathbb{R}_{+} \times \Omega \times U \rightarrow \mathbb{R}^{d}$ is called $\left(\mathscr{F}_{t}\right)_{t \in \mathbb{R}_{+}}{ }^{-}$ predictable if it is $\mathscr{P} / \mathscr{B}\left(\mathbb{R}^{d}\right)$-measurable.

Lemma A.1. Let $\left(\Omega, \mathscr{F},\left(\mathscr{F}_{t}\right)_{t \in \mathbb{R}_{+}}, \mathbb{P}\right)$ be a filtered probability space. Let $\left(\mathbf{X}_{t}\right)_{t \in \mathbb{R}_{+}}$be an $\left(\mathscr{F}_{t}\right)_{t \in \mathbb{R}_{+}}$-adapted càdlàg process with values in $\mathbb{R}^{d}$. (i) If $w: \mathbb{R}^{d} \rightarrow \mathbb{R}$ is a continuous function, then for each $T \in \mathbb{R}_{+}$and $B \in \mathscr{B}(U)$, the function $h(t, \omega, u):=$ $w\left(\mathbf{X}_{t-}(\omega)\right) \mathbb{1}_{[0, T]}(t) \mathbb{1}_{B}(u),(t, \omega, u) \in \mathbb{R}_{+} \times \Omega \times U$, is $\left(\mathscr{F}_{t}\right)_{t \in \mathbb{R}_{+}}$-predictable.

(ii) If $T \in \mathbb{R}_{+}, A \in \mathscr{B}\left(\mathbb{R}^{d}\right)$ is an open set and $B \in \mathscr{B}(U)$, then

$$
\begin{aligned}
& \left\{(t, \omega, u) \in \mathbb{R}_{+} \times \Omega \times U:\right. \\
& \left.\quad t \in[0, T], \mathbf{X}_{t-}(\omega) \in A, u \in B\right\} \in \mathscr{P} .
\end{aligned}
$$

(iii) If $f: \mathbb{R}_{+} \times \mathbb{R}^{d} \times U \rightarrow \mathbb{R}^{d}$ is $\mathscr{B}\left(\mathbb{R}_{+}\right) \otimes \mathscr{B}\left(\mathbb{R}^{d}\right) \otimes \mathscr{B}(U) /$ $\mathscr{B}\left(\mathbb{R}^{d}\right)$-measurable, then the function $H(t, \omega, u):=$ $f\left(t, \mathbf{X}_{t-}(\omega), u\right),(t, \omega, u) \in \mathbb{R}_{+} \times \Omega \times U$, is $\left(\mathscr{F}_{t}\right)_{t \in \mathbb{R}_{+}}{ }^{-}$ predictable.

Proof. (i) The function $h$ is $\left(\mathscr{F}_{t}\right)_{t \in \mathbb{R}_{+}}$-predictable, since it belongs to the generator system of $\mathscr{P}$. Indeed, for each $t \in \mathbb{R}_{+}$, the mapping $\Omega \times U \ni(\omega, u) \mapsto h(t, \omega, u)$ is $\mathscr{F}_{t} \otimes \mathscr{B}(U) / \mathscr{B}(\mathbb{R})$-measurable, because $\mathbf{X}_{s}$ is $\mathscr{F}_{s} / \mathscr{B}\left(\mathbb{R}^{d}\right)$ measurable and $\mathscr{F}_{s} \subset \mathscr{F}_{t}$ for all $s<t$, and hence $\mathbf{X}_{t-}:=$ $\lim _{s \uparrow t} \mathbf{X}_{s}$ is $\mathscr{F}_{t} / \mathscr{B}\left(\mathbb{R}^{d}\right)$-measurable, and $w$ is $\mathscr{B}\left(\mathbb{R}^{d}\right) / \mathscr{B}(\mathbb{R})$ measurable. Moreover, for each $(\omega, u) \in \Omega \times U$, the function $\mathbb{R}_{+} \ni t \mapsto h(t, \omega, u)$ is left continuous, because the functions $\mathbb{R}_{+} \ni t \mapsto \mathbb{1}_{[0, T]}(t)$ and $\mathbb{R}_{+} \ni t \mapsto \mathbf{X}_{t-}(\omega)$ are left continuous and $w$ is continuous.

(ii) Consider the function $w_{A}: \mathbb{R}^{d} \rightarrow \mathbb{R}_{+}$given by $w_{A}(\mathbf{x}):=\varrho\left(\mathbf{x}, \mathbb{R}^{d} \backslash A\right), \mathbf{x} \in \mathbb{R}^{d}$, where $\varrho$ denotes the Euclidean distance of $\mathbf{x}$ and $\mathbb{R}^{d} \backslash A$. Then $w_{A}$ is continuous and $A=$ $w_{A}^{-1}\left(\mathbb{R}_{++}\right)$. Put $h_{A}(t, \omega, u):=w_{A}\left(\mathbf{X}_{t-}(\omega)\right) \mathbb{1}_{[0, T]}(t) \mathbb{1}_{B}(u),(t, \omega$, u) $\in \mathbb{R}_{+} \times \Omega \times U$. Then, by (i), we obtain

$$
\begin{aligned}
& \left\{(t, \omega, u) \in \mathbb{R}_{+} \times \Omega \times U: t \in[0, T], \mathbf{X}_{t-}(\omega) \in A, u \in B\right\} \\
& =\left\{(t, \omega, u) \in \mathbb{R}_{+} \times \Omega \times U:\right. \\
& \left.\quad t \in[0, T], w_{A}\left(\mathbf{X}_{t-}(\omega)\right) \in \mathbb{R}_{++}, u \in B\right\} \\
& =\left\{(t, \omega, u) \in \mathbb{R}_{+} \times \Omega \times U: h_{A}(t, \omega, u) \in \mathbb{R}_{++}\right\} \in \mathscr{P} .
\end{aligned}
$$

(iii) We have $H=f \circ G$, where $G(t, \omega, u):=\left(t, \mathbf{X}_{t-}(\omega), u\right)$, $(t, \omega, u) \in \mathbb{R}_{+} \times \Omega \times U$; thus it suffices to show that $G$ is $\mathscr{P} / \mathscr{B}\left(\mathbb{R}_{+}\right) \otimes \mathscr{B}\left(\mathbb{R}^{d}\right) \otimes \mathscr{B}(U)$-measurable. The $\sigma$-algebra $\mathscr{B}\left(\mathbb{R}_{+}\right) \otimes \mathscr{B}\left(\mathbb{R}^{d}\right) \otimes \mathscr{B}(U)$ is generated by the sets $[0, T] \times A \times B$ with $T \in \mathbb{R}_{+}$, open sets $A \in \mathscr{B}\left(\mathbb{R}^{d}\right)$ and $B \in \mathscr{B}(U)$; hence it suffices to show that

$$
\begin{aligned}
& \left\{(t, \omega, u) \in \mathbb{R}_{+} \times \Omega \times U:\right. \\
& \left.\quad t \in[0, T], \mathbf{X}_{t-}(\omega) \in A, u \in B\right\} \in \mathscr{P} .
\end{aligned}
$$

This holds by (ii).

Note that using Lemma A.1, one can relax Assumption 6.2.8 in Applebaum [23]. [11].

The next lemma plays a similar role as Lemma 139 in Situ

Lemma A.2. Let $\left(\Omega^{(i)}, \mathscr{F}^{(i)},\left(\mathscr{F}_{t}^{(i)}\right)_{t \in \mathbb{R}}, \mathbb{P}^{(i)}, \mathbf{W}^{(i)}, p^{(i)}, \mathbf{X}^{(i)}\right)$, $i \in\{1,2\}$, be tuples satisfying (D1), (D2), (D3), and (D4)(b)(d). Suppose that $\left(\mathbf{W}^{(1)}, p^{(1)}, \mathbf{X}^{(1)}\right)$ and $\left(\mathbf{W}^{(2)}, p^{(2)}, \mathbf{X}^{(2)}\right)$ have 
the same distribution on $C\left(\mathbb{R}_{+}, \mathbb{R}^{r}\right) \times M\left(\mathbb{R}_{+} \times U\right) \times D\left(\mathbb{R}_{+}, \mathbb{R}^{d}\right)$. Then

$$
\begin{gathered}
\left(\mathbf{X}_{t}^{(1)}, \int_{0}^{t} b\left(s, \mathbf{X}_{s}^{(1)}\right) \mathrm{d} s, \int_{0}^{t} \sigma\left(s, \mathbf{X}_{s}^{(1)}\right) \mathrm{d} \mathbf{W}_{s}^{(1)},\right. \\
\int_{0}^{t} \int_{U_{0}} f\left(s, \mathbf{X}_{s-}^{(1)}, u\right) \widetilde{N}^{(1)}(\mathrm{d} s, \mathrm{~d} u), \\
\left.\int_{0}^{t} \int_{U_{1}} g\left(s, \mathbf{X}_{s-}^{(1)}, u\right) N^{(1)}(\mathrm{d} s, \mathrm{~d} u)\right)_{t \in \mathbb{R}_{+}}, \\
\left(\mathbf{X}_{t}^{(2)}, \int_{0}^{t} b\left(s, \mathbf{X}_{s}^{(2)}\right) \mathrm{d} s, \int_{0}^{t} \sigma\left(s, \mathbf{X}_{s}^{(2)}\right) \mathrm{d}_{\mathbf{W}_{s}^{(2)}},\right. \\
\int_{0}^{t} \int_{U_{0}} f\left(s, \mathbf{X}_{s-}^{(2)}, u\right) \widetilde{N}^{(2)}(\mathrm{d} s, \mathrm{~d} u), \\
\left.\int_{0}^{t} \int_{U_{1}} g\left(s, \mathbf{X}_{s-}^{(2)}, u\right) N^{(2)}(\mathrm{d} s, \mathrm{~d} u)\right)_{t \in \mathbb{R}_{+}}
\end{gathered}
$$

have the same distribution on $\left(D\left(\mathbb{R}_{+}, \mathbb{R}^{d}\right)\right)^{5}$, where, for each $i \in$ $\{1,2\}, N^{(i)}(\mathrm{d} s, \mathrm{~d} u)$ is the counting measure of $p^{(i)}$ on $\mathbb{R}_{++} \times U$, and $\widetilde{N}^{(i)}(\mathrm{d} s, \mathrm{~d} u):=N^{(i)}(\mathrm{d} s, \mathrm{~d} u)-\mathrm{d} s m(\mathrm{~d} u)$.

Proof. By Remark 4, the above processes have càdlàg modifications. According to Lemma VI.3.19 in Jacod and Shiryaev [17], it suffices to show that the finite-dimensional distributions of the above processes coincide.

By Proposition I.4.44 in Jacod and Shiryaev [17], for each $i \in\{1,2\}$ and $t \in \mathbb{R}_{+}, I_{1, n}^{(i)}(t) \stackrel{\mathbb{P}^{(i)}}{\longrightarrow} \int_{0}^{t} b\left(s, \mathbf{X}_{s}^{(i)}\right) \mathrm{d} s$ and $I_{2, n}^{(i)}(t) \stackrel{\mathbb{P}^{(i)}}{\longrightarrow}$ $\int_{0}^{t} \sigma\left(s, \mathbf{X}_{s}^{(i)}\right) \mathrm{d} \mathbf{W}_{s}^{(i)}$ as $n \rightarrow \infty$, where

$$
\begin{gathered}
I_{1, n}^{(i)}(t):=\frac{1}{n} \sum_{k=1}^{\lfloor n t\rfloor} b\left(\frac{k-1}{n}, \mathbf{X}_{(k-1) / n}^{(i)}\right), \\
I_{2, n}^{(i)}(t):=\sum_{k=1}^{\lfloor n t\rfloor} \sigma\left(\frac{k-1}{n}, \mathbf{X}_{(k-1) / n}^{(i)}\right)\left(\mathbf{W}_{k / n}^{(i)}-\mathbf{W}_{(k-1) / n}^{(i)}\right) .
\end{gathered}
$$

Let $U_{1, j} \in \mathscr{B}(U), j \in \mathbb{N}$, be such that they are disjoint, $m\left(U_{1, j}\right)<\infty, j \in \mathbb{N}$, and $U_{1}=\bigcup_{j=1}^{\infty} U_{1, j}$ (such a sequence exists since $m$ is $\sigma$-finite; see, e.g., Cohn [22, page 9]). Then for each $i \in\{1,2\}$ and $t \in \mathbb{R}_{+}, I_{3, n}^{(i)}(t) \rightarrow \int_{0}^{t} \int_{U_{1}} g\left(s, \mathbf{X}_{s^{-}}^{(i)}\right.$, $u) N^{(i)}(\mathrm{d} s, \mathrm{~d} u)$ as $n \rightarrow \infty \mathbb{P}^{(i)}$-almost surely, where

$$
\begin{aligned}
I_{3, n}^{(i)}(t) & :=\sum_{j=1}^{n} \int_{0}^{t} \int_{U_{1, j}} g\left(s, \mathbf{X}_{s-}^{(i)}, u\right) N^{(i)}(\mathrm{d} s, \mathrm{~d} u) \\
& =\sum_{j=1}^{n} \sum_{s \in(0, t] \cap D\left(p_{1, j}^{(i)}\right)} g\left(s, \mathbf{X}_{s^{-}}^{(i)}, p_{1, j}^{(i)}(s)\right),
\end{aligned}
$$

where $p_{1, j}^{(i)}$ denotes the thinning of $p^{(i)}$ onto $U_{1, j}$; see, for example, Ikeda and Watanabe [20, page 62]. Since $m\left(U_{1, j}\right)<$ $\infty$, by Remark 5 , the set $(0, t] \cap D\left(p_{1, j}^{(i)}\right)$ is finite $\mathbb{P}^{(i)}$-almost surely for all $t \in \mathbb{R}_{+}$and $i \in\{1,2\}, j \in \mathbb{N}$, and hence one can order the set $D\left(p_{1, j}^{(i)}\right)$ according to magnitude, say $0<\zeta_{1, j, 1}^{(i)}<$ $\zeta_{1, j, 2}^{(i)}<\cdots, j \in \mathbb{N}, i \in\{1,2\}$. Namely,

$$
\begin{array}{r}
\zeta_{1, j, \ell}^{(i)}=\inf \left\{t \in \mathbb{R}_{+}: N^{(i)}\left((0, t] \times U_{1, j}\right) \geqslant \ell\right\}, \\
\ell \in \mathbb{N}, \quad j \in \mathbb{N}, \quad i \in\{1,2\}
\end{array}
$$

on the event

$$
\begin{array}{r}
\Omega_{1, j}^{(i)}:=\bigcap_{k=1}^{\infty}\left\{\omega \in \Omega^{(i)}: N_{p_{1, j}^{(i)}(\omega)}\left((0, k] \times U_{1, j}\right)<\infty\right\}, \\
j \in \mathbb{N}, \quad i \in\{1,2\},
\end{array}
$$

having $\mathbb{P}^{(i)}$-probability 1 , where we used that the point measure corresponding to the point function $p_{1, j}^{(i)}(\omega)$ is its counting measure $N_{p_{1, j}^{(i)}(\omega)}$; see Section 2 . Then we can write $I_{3, n}^{(i)}(t)$ in the form

$$
\begin{array}{r}
I_{3, n}^{(i)}(t)=\sum_{j=1}^{n} \sum_{\ell=1}^{\infty} g\left(\zeta_{1, j, \ell}^{(i)}, \mathbf{X}_{\zeta_{1, j, \ell^{-}}^{(i)}}^{(i)}, p_{1, j}^{(i)}\left(\zeta_{1, j, \ell}^{(i)}\right)\right) \mathbb{1}_{(0, t]}\left(\zeta_{1, j, \ell}^{(i)}\right) \\
t \in \mathbb{R}_{+}, \quad n \in \mathbb{N}, \quad i \in\{1,2\}
\end{array}
$$

where $\sum_{\ell=1}^{\infty} g\left(\zeta_{1, j, \ell}^{(i)}, \mathbf{X}_{\zeta_{1, j, \ell^{-}}^{(i)}}^{(i)}, p_{1, j}^{(i)}\left(\zeta_{1, j, \ell}^{(i)}\right)\right) \mathbb{1}_{(0, t]}\left(\zeta_{1, j, \ell}^{(i)}\right)$ is a finite sum $\mathbb{P}^{(i)}$-almost surely. Furthermore, by Remark 4, for $i \epsilon$ $\{1,2\}$ and $t \in \mathbb{R}_{+}, I_{4, n}^{(i)}(t) \rightarrow \int_{0}^{t} \int_{U_{0}} f\left(s, \mathbf{X}_{s-}^{(i)}, u\right) \widetilde{N}^{(i)}(\mathrm{d} s, \mathrm{~d} u)$ as $n \rightarrow \infty \mathbb{P}^{(i)}$-almost surely, where

$$
I_{4, n}^{(i)}(t):=\int_{0}^{t} \int_{U_{0}} \mathbb{1}_{\left[0, \tau_{n}^{(i)}\right]}(s) f\left(s, \mathbf{X}_{s-}^{(i)}, u\right) \widetilde{N}^{(i)}(\mathrm{d} s, \mathrm{~d} u)
$$

with

$$
\begin{aligned}
& \tau_{n}^{(i)} \\
& :=\inf \left\{t \in \mathbb{R}_{+}: \int_{0}^{t} \int_{U_{0}}\left\|f\left(s, \mathbf{X}_{s}^{(i)}, u\right)\right\|^{2} \mathrm{~d} s m(\mathrm{~d} u) \geqslant n\right\} \wedge n,
\end{aligned}
$$

for all $n \in \mathbb{N}, i \in\{1,2\}$, satisfying $\tau_{n}^{(i)} \uparrow \infty \mathbb{P}^{(i)}$-almost surely as $n \rightarrow \infty$. Let $U_{0, j} \in \mathscr{B}(U), j \in \mathbb{N}$, be such that they are disjoint, $m\left(U_{0, j}\right)<\infty, j \in \mathbb{N}$, and $U_{0}=\bigcup_{j=1}^{\infty} U_{0, j}$ (such a sequence exists since $m$ is $\sigma$-finite; see, e.g., Cohn [22, page 9]). Then, by pages 47 and 63 in Ikeda and Watanabe [20], for all $t \in \mathbb{R}_{+}, i \in\{1,2\}$ and $n \in \mathbb{N}, I_{4, n, j}^{(i)}(t) \stackrel{\mathbb{P}^{(i)}}{\longrightarrow} I_{4, n}^{(i)}(t)$ as $j \rightarrow \infty$, where

$$
\begin{aligned}
& I_{4, n, j}^{(i)}(t):=\int_{0}^{t} \int_{U_{0}} \mathbb{1}_{(-j, j)}\left(\mathbb{1}_{\left[0, \tau_{n}^{(i)}\right]}(s) f\left(s, \mathbf{X}_{s-}^{(i)}, u\right)\right) \\
& \times \mathbb{1}_{U_{0, j}}(u) \mathbb{1}_{\left[0, \tau_{n}^{(i)}\right]}(s) f\left(s, \mathbf{X}_{s-}^{(i)}, u\right) \\
& \widetilde{N}^{(i)}(\mathrm{d} s, \mathrm{~d} u)
\end{aligned}
$$




$$
\begin{aligned}
& =\int_{0}^{t} \int_{U_{0, j}} \mathbb{1}_{(-j, j)}\left(f\left(s, \mathbf{X}_{s-}^{(i)}, u\right)\right) \\
& \quad \times \mathbb{1}_{\left[0, \tau_{n}^{(i)}\right]}(s) f\left(s, \mathbf{X}_{s-}^{(i)}, u\right) \widetilde{N}^{(i)}(\mathrm{d} s, \mathrm{~d} u) .
\end{aligned}
$$

By page 62 in Ikeda and Watanabe [20], for all $t \in \mathbb{R}_{+}, i \in$ $\{1,2\}, n \in \mathbb{N}$, and $j \in \mathbb{N}, I_{4, n, j}^{(i)}(t)=I_{4, n, j}^{(i), a}(t)-I_{4, n, j}^{(i), b}(t)$, where

$$
\begin{aligned}
& I_{4, n, j}^{(i), a}(t):= \int_{0}^{t} \int_{U_{0, j}} \mathbb{1}_{(-j, j)}\left(f\left(s, \mathbf{X}_{s-}^{(i)}, u\right)\right) \\
& \times \mathbb{1}_{\left[0, \tau_{n}^{(i)}\right]}(s) f\left(s, \mathbf{X}_{s-}^{(i)}, u\right) N^{(i)}(\mathrm{d} s, \mathrm{~d} u) \\
& I_{4, n, j}^{(i), b}(t):=\int_{0}^{t}\left(\int_{U_{0, j}} \mathbb{1}_{(-j, j)}\left(f\left(s, \mathbf{X}_{s-}^{(i)}, u\right)\right) \mathbb{1}_{\left[0, \tau_{n}^{(i)}\right]}(s)\right. \\
&\left.\quad \times f\left(s, \mathbf{X}_{s-}^{(i)}, u\right) m(\mathrm{~d} u)\right) \mathrm{d} s .
\end{aligned}
$$

Similarly as for the integrals $\int_{0}^{t} \int_{U_{1}} g\left(s, \mathbf{X}_{s-}^{(i)}, u\right) N^{(i)}(\mathrm{d} s, \mathrm{~d} u)$ and $\int_{0}^{t} b\left(s, \mathbf{X}_{s}^{(i)}\right) \mathrm{d} s$, there exist sequences of random variables $\left(I_{4, n, j, \ell}^{(i), a}(t)\right)_{\ell \in \mathbb{N}}$ and $\left(I_{4, n, j, \ell}^{(i), b}(t)\right)_{\ell \in \mathbb{N}}$ such that $I_{4, n, j, \ell}^{(i), a}(t) \stackrel{\mathbb{P}^{(i)}}{\longrightarrow}$ $I_{4, n, j}^{(i), a}(t)$ and $I_{4, n, j, \ell}^{(i), b}(t) \stackrel{\mathbb{P}^{(i)}}{\longrightarrow} I_{4, n, j}^{(i), b}(t)$ as $\ell \rightarrow \infty$, respectively. Then, for all $t \in \mathbb{R}_{+}$and $i \in\{1,2\}, I_{4, n, j, \ell}^{(i), a}(t)-I_{4, n, j, \ell}^{(i), b}(t) \stackrel{\mathbb{P}^{(i)}}{\longrightarrow}$ $\int_{0}^{t} \int_{U_{0}} f\left(s, \mathbf{X}_{s-}^{(i)}, u\right) \widetilde{N}^{(i)}(\mathrm{d} s, \mathrm{~d} u)$ as $\ell \rightarrow \infty$; then $j \rightarrow \infty$, and, finally, $n \rightarrow \infty$. Using part (vi) of Theorem 2.7 in van der Vaart [24], we get for all $K \in \mathbb{N}, t_{1}, \ldots, t_{K} \in \mathbb{R}_{+}$and $i \in\{1,2\}$,

$$
\begin{gathered}
\left(\mathbf{X}_{t_{k}}^{(i)}, I_{1, n}^{(i)}\left(t_{k}\right), I_{2, n}^{(i)}\left(t_{k}\right), I_{3, n}^{(i)}\left(t_{k}\right), I_{4, n, j, \ell}^{(i), a}\left(t_{k}\right)-I_{4, n, j, \ell}^{(i), b}\left(t_{k}\right)\right)_{k \in\{1, \ldots, K\}} \\
\stackrel{\mathbb{P}^{(i)}}{\longrightarrow}\left(\mathbf{X}_{t_{k}}^{(i)}, \int_{0}^{t_{k}} b\left(s, \mathbf{X}_{s}^{(i)}\right) \mathrm{d} s, \int_{0}^{t_{k}} \sigma\left(s, \mathbf{X}_{s}^{(i)}\right) \mathrm{d} \mathbf{W}_{s}^{(i)},\right. \\
\quad \int_{0}^{t_{k}} \int_{U_{1}} g\left(s, \mathbf{X}_{s-}^{(i)}, u\right) N^{(i)}(\mathrm{d} s, \mathrm{~d} u), \\
\left.\quad \int_{0}^{t_{k}} \int_{U_{0}} f\left(s, \mathbf{X}_{s-}^{(i)}, u\right) \widetilde{N}^{(i)}(\mathrm{d} s, \mathrm{~d} u)\right)_{k \in\{1, \ldots, K\}}
\end{gathered}
$$

as $\ell, j, n \rightarrow \infty$. Since $\left(\mathbf{W}^{(1)}, p^{(1)}, \mathbf{X}^{(1)}\right)$ and $\left(\mathbf{W}^{(2)}, p^{(2)}, \mathbf{X}^{(2)}\right)$ have the same distribution, the random vectors

$$
\begin{aligned}
& \left(\mathbf{X}_{t_{k}}^{(1)}, I_{1, n}^{(1)}\left(t_{k}\right), I_{2, n}^{(1)}\left(t_{k}\right), I_{3, n}^{(1)}\left(t_{k}\right), I_{4, n, j, \ell}^{(1), a}\left(t_{k}\right)-I_{4, n, j, \ell}^{(1), b}\left(t_{k}\right)\right)_{k \in\{1, \ldots, K\}}, \\
& \left(\mathbf{X}_{t_{k}}^{(2)}, I_{1, n}^{(2)}\left(t_{k}\right), I_{2, n}^{(2)}\left(t_{k}\right), I_{3, n}^{(2)}\left(t_{k}\right), I_{4, n, j, \ell}^{(2), a}\left(t_{k}\right)-I_{4, n, j, \ell}^{(2), b}\left(t_{k}\right)\right)_{k \in\{1, \ldots, K\}}
\end{aligned}
$$

have the same distribution for all $\ell, j, n \in \mathbb{N}$, as well. Indeed, the random vectors above can be considered as some appropriate measurable function of $\left(\mathbf{W}^{(1)}, p^{(1)}, \mathbf{X}^{(1)}\right)$ and $\left(\mathbf{W}^{(2)}, p^{(2)}, \mathbf{X}^{(2)}\right)$, respectively. For this, it is enough to verify that each coordinate of the above random vectors can be considered as some appropriate measurable function of $\left(\mathbf{W}^{(1)}, p^{(1)}, \mathbf{X}^{(1)}\right)$ and $\left(\mathbf{W}^{(2)}, p^{(2)}, \mathbf{X}^{(2)}\right)$, respectively, hence we fix $k \in\{1, \ldots, K\}$.

(i) First observe that $\mathbf{X}_{t_{k}}^{(i)}$ is a $\mathscr{D}\left(\mathbb{R}_{+}, \mathbb{R}^{d}\right) / \mathscr{B}\left(\mathbb{R}^{d}\right)$-measurable function of $\mathbf{X}^{(i)}$; namely, $\mathbf{X}_{t_{k}}^{(i)}=\Psi_{0}\left(\mathbf{X}^{(i)}\right)$, where $\Psi_{0}: D\left(\mathbb{R}_{+}, \mathbb{R}^{d}\right) \rightarrow \mathbb{R}^{d}$ is given by $\Psi_{0}(y):=y\left(t_{k}\right), y \in$ $D\left(\mathbb{R}_{+}, \mathbb{R}^{d}\right)$.

(ii) Next, $I_{1, n}^{(i)}\left(t_{k}\right)$ is a $\mathscr{D}\left(\mathbb{R}_{+}, \mathbb{R}^{d}\right) / \mathscr{B}\left(\mathbb{R}^{d}\right)$-measurable function of $\mathbf{X}^{(i)}$ as well; namely, $I_{1, n}^{(i)}\left(t_{k}\right)=\Psi_{1}\left(\mathbf{X}^{(i)}\right)$, where $\Psi_{1}: D\left(\mathbb{R}_{+}, \mathbb{R}^{d}\right) \rightarrow \mathbb{R}^{d}$ is given by $\Psi_{1}(y):=(1 /$ n) $\sum_{k=1}^{\left\lfloor n t_{k}\right\rfloor} b((k-1) / n, y((k-1) / n)), y \in D\left(\mathbb{R}_{+}, \mathbb{R}^{d}\right)$.

(iii) In a similar way, $I_{2, n}^{(i)}\left(t_{k}\right)$ is a $\mathscr{D}\left(\mathbb{R}_{+}, \mathbb{R}^{d}\right) \times \mathscr{C}\left(\mathbb{R}_{+}, \mathbb{R}^{r}\right) /$ $\mathscr{B}\left(\mathbb{R}^{d}\right)$-measurable function of $\left(\mathbf{X}^{(i)}, \mathbf{W}^{(i)}\right)$; namely, $I_{2, n}^{(i)}\left(t_{k}\right)=\Psi_{2}\left(\mathbf{X}^{(i)}, \mathbf{W}^{(i)}\right)$, where $\Psi_{2}: D\left(\mathbb{R}_{+}, \mathbb{R}^{d}\right) \times$ $C\left(\mathbb{R}_{+}, \mathbb{R}^{r}\right) \rightarrow \mathbb{R}^{d}$ is given by $\Psi_{2}(y, w):=\sum_{k=1}^{\left\lfloor n t_{k}\right\rfloor} \sigma((k-$ $1) / n, y((k-1) / n))(w(k / n)-w((k-1) / n)), y \in D\left(\mathbb{R}_{+}\right.$, $\left.\mathbb{R}^{d}\right), w \in C\left(\mathbb{R}_{+}, \mathbb{R}^{r}\right)$.

(iv) Now we show that $I_{3, n}^{(i)}\left(t_{k}\right)$ is a $\mathscr{D}\left(\mathbb{R}_{+}, \mathbb{R}^{d}\right) \otimes \mathscr{M}\left(\mathbb{R}_{+} \times\right.$ $U) / \mathscr{B}\left(\mathbb{R}^{d}\right)$-measurable function of $\left(\mathbf{X}^{(i)}, p^{(i)}\right)$. As a first step, we show that for each $j, \ell \in \mathbb{N}$ there exist functions $\Phi_{j, \ell}: M\left(\mathbb{R}_{+} \times U\right) \rightarrow \mathbb{R}_{+}$and $\Xi_{j, \ell}: M\left(\mathbb{R}_{+} \times\right.$ $U) \rightarrow U$ such that $\Phi_{j, \ell}$ is $\mathscr{M}\left(\mathbb{R}_{+} \times U\right) / \mathscr{B}\left(\mathbb{R}_{+}\right)$measurable, $\Xi_{j, \ell}$ is $\mathscr{M}\left(\mathbb{R}_{+} \times U\right) / \mathscr{B}(U)$-measurable, and $\left(\zeta_{1, j, \ell}^{(i)}, p_{1, j}^{(i)}\left(\zeta_{1, j, \ell}^{(i)}\right)\right)=\left(\Phi_{j, \ell}\left(N_{p_{1, j}^{(i)}}\right), \Xi_{j, \ell}\left(N_{p_{1, j}^{(i)}}\right)\right)$ holds $\mathbb{P}^{(i)}$-almost surely. Then it will follow that $I_{3, n}^{(i)}\left(t_{k}\right)=$ $\Psi_{3}\left(\mathbf{X}^{(i)}, p^{(i)}\right)$, where $\Psi_{3}: D\left(\mathbb{R}_{+}, \mathbb{R}^{d}\right) \times M\left(\mathbb{R}_{+} \times U\right) \rightarrow$ $\mathbb{R}^{d}$ given by

$\Psi_{3}(y, \pi)$

$$
:=\sum_{j=1}^{n} \sum_{\ell=1}^{\infty} g\left(\Phi_{j, \ell}(\pi), y\left(\Phi_{j, \ell}(\pi)-\right), \Xi_{j, \ell}(\pi)\right) \mathbb{1}_{\left(0, t_{k}\right]}\left(\Phi_{j, \ell}(\pi)\right)
$$

for $(y, \pi) \in D\left(\mathbb{R}_{+}, \mathbb{R}^{d}\right) \times M\left(\mathbb{R}_{+} \times U\right)$ is $\mathscr{D}\left(\mathbb{R}_{+}, \mathbb{R}^{d}\right) \otimes$ $\mathscr{M}\left(\mathbb{R}_{+} \times U\right) / \mathscr{B}\left(\mathbb{R}^{d}\right)$-measurable. To prove the existence of $\Phi_{j, \ell}$ and $\Xi_{j, \ell}$, first we verify that $\left(\zeta_{1, j, \ell}^{(i)}\right.$, $\left.p_{1, j}^{(i)}\left(\zeta_{1, j, \ell}^{(i)}\right)\right)$ is measurable with respect to the $\sigma$-algebra $\sigma\left(N_{p_{1, j}^{(i)}}\right) \cap \Omega_{1, j}^{(i)}$ having the form

$$
\begin{gathered}
\sigma\left(\left\{\omega \in \Omega_{1, j}^{(i)}: N_{p_{1, j}^{(i)}(\omega)}((0, t] \times B)=k\right\} \mid\right. \\
\left.t \in \mathbb{R}_{+}, B \in \mathscr{B}\left(U_{1, j}\right), k \in \mathbb{N}\right) .
\end{gathered}
$$


We have

$$
\begin{gathered}
\left\{\omega \in \Omega_{1, j}^{(i)}:\left(\zeta_{1, j, \ell}^{(i)}(\omega), p_{1, j}^{(i)}(\omega)\left(\zeta_{1, j, \ell}^{(i)}(\omega)\right)\right) \in(0, t] \times B\right\} \\
=\bigcap_{n=1}^{\infty} \bigcup_{k=1}^{n}\left\{\omega \in \Omega_{1, j}^{(i)}:\right. \\
N_{p_{1, j}^{(i)}(\omega)}\left(\left(0, \frac{(k-1) t}{n}\right] \times U_{1, j}\right) \leqslant \ell-1, \\
N_{p_{1, j}^{(i)}(\omega)}\left(\left(\frac{(k-1) t}{n}, \frac{k t}{n}\right] \times B\right) \geqslant 1, \\
\left.N_{p_{1, j}^{(i)}(\omega)}\left(\left(0, \frac{k t}{n}\right] \times U_{1, j}\right) \geqslant \ell\right\}
\end{gathered}
$$

for $t \in \mathbb{R}_{++}, j, \ell \in \mathbb{N}, B \in \mathscr{B}\left(U_{1, j}\right), i \in\{1,2\}$. Indeed, on the one hand, if $\omega \in \Omega_{1, j}^{(i)}$ is such that $\zeta_{1, j, \ell}^{(i)}(\omega) \in$ $(0, t]$ and $p_{1, j}^{(i)}(\omega)\left(\zeta_{1, j, \ell}^{(i)}(\omega)\right) \in B$, then for each $n \in \mathbb{N}$, there exists a unique $k \in\{1, \ldots, n\}$ with $\zeta_{1, j, \ell}^{(i)}(\omega) \epsilon$ $((k-1) t / n, k t / n]$, and hence $N_{p_{1, j}^{(i)}(\omega)}((0,(k-1) t / n] \times$ $\left.U_{1, j}\right) \leqslant \ell-1, N_{p_{1, j}^{(i)}(\omega)}(((k-1) t / n, k t / n] \times B) \geqslant 1$ and $N_{p_{1, j}^{(i)}(\omega)}\left((0, k t / n] \times U_{1, j}\right) \geqslant \ell$. On the other hand,

$\left\{\omega \in \Omega_{1, j}^{(i)}: \zeta_{1, j, \ell}^{(i)}(\omega) \notin(0, t]\right\}$

$$
\begin{aligned}
= & \left\{\omega \in \Omega_{1, j}^{(i)}: N_{p_{1, j}^{(i)}(\omega)}\left((0, t] \times U_{1, j}\right) \leqslant \ell-1\right\} \\
& \subset \bigcup_{n=1}^{\infty} \bigcap_{k=1}^{n}\left\{\omega \in \Omega_{1, j}^{(i)}: N_{p_{1, j}^{(i)}(\omega)}\left(\left(0, \frac{k t}{n}\right] \times U_{1, j}\right) \leqslant \ell-1\right\},
\end{aligned}
$$

$$
\left\{\omega \in \Omega_{1, j}^{(i)}: \zeta_{1, j, \ell}^{(i)}(\omega) \in(0, t], p_{1, j}^{(i)}(\omega)\left(\zeta_{1, j, \ell}^{(i)}(\omega)\right) \notin B\right\}
$$

$$
\begin{gathered}
\subset \bigcup_{n=1}^{\infty} \bigcap_{k=1}^{n}\left(\left\{\omega \in \Omega_{1, j}^{(i)}: N_{p_{1, j}^{(i)}(\omega)}\left(\left(0, \frac{(k-1) t}{n}\right] \times U_{1, j}\right) \geqslant \ell\right\}\right. \\
\cup\left\{\omega \in \Omega_{1, j}^{(i)}: N_{p_{1, j}^{(i)}(\omega)}\left(\left(\frac{(k-1) t}{n}, \frac{k t}{n}\right] \times B\right)=0\right\} \\
\cup\left\{\omega \in \Omega_{1, j}^{(i)}:\right. \\
\left.\left.\quad N_{p_{1, j}^{(i)}(\omega)}\left(\left(0, \frac{k t}{n}\right] \times U_{1, j}\right) \leqslant \ell-1\right\}\right) .
\end{gathered}
$$

For the second inclusion, for each $\omega \in \Omega_{1, j}^{(i)}$, let us choose $n(\omega) \in \mathbb{N}$ such that

$n(\omega)$

$$
>\max \left(\frac{1}{\zeta_{1, j, \ell}^{(i)}(\omega)-\zeta_{1, j, \ell-1}^{(i)}(\omega)}, \frac{1}{\zeta_{1, j, \ell+1}^{(i)}(\omega)-\zeta_{1, j, \ell}^{(i)}(\omega)}\right) .
$$

If $\omega \in \Omega_{1, j}^{(i)}$ is such that $\zeta_{1, j, \ell}^{(i)}(\omega) \in(0, t]$ and $p_{1, j}^{(i)}(\omega)\left(\zeta_{1, j, \ell}^{(i)}(\omega)\right) \notin B$, then there exists a unique $k^{*} \epsilon$ $\{1, \ldots, n\}$ with $\zeta_{1, j, \ell}^{(i)}(\omega) \in\left(\left(k^{*}-1\right) t / n, k^{*} t / n\right]$, and hence we have $N_{p_{1, j}^{(i)}(\omega)}\left((0, k t / n] \times U_{1, j}\right) \leqslant \ell-1$ for $k \epsilon$ $\left\{1, \ldots, k^{*}-1\right\}, N_{p_{1, j}^{(i)}(\omega)}\left(\left(\left(k^{*}-1\right) t / n, k^{*} t / n\right] \times B\right)=0$, and $N_{p_{1, j}^{(i)}(\omega)}\left((0,(k-1) t / n] \times U_{1, j}\right) \geqslant \ell$ for $k \in\left\{k^{*}+1, \ldots, n\right\}$.

Since the set on right hand side of (A.21) is in the $\sigma$ algebra given in (A.20) and $\left\{(0, t] \times B: t \in \mathbb{R}_{+}, B \in\right.$ $\left.\mathscr{B}\left(U_{1, j}\right)\right\}$ is a generator system of $\mathscr{B}\left(\mathbb{R}_{+}\right) \otimes \mathscr{B}\left(U_{1, j}\right)$, we readily get that the random variable $\left(\zeta_{1, j, \ell}^{(i)}, p_{1, j}^{(i)}\left(\zeta_{1, j, \ell}^{(i)}\right)\right)$ is measurable with respect to the $\sigma$-algebra given in (A.20). Let us apply Theorem 4.2.8 in Dudley [2] with the following choices:

(a) $X:=\Omega_{1, j}^{(i)}, Y:=M\left(\mathbb{R}_{+} \times U\right)$,

(b) $T: \Omega_{1, j}^{(i)} \rightarrow M\left(\mathbb{R}_{+} \times U\right), T(\omega):=N_{p_{1, j}^{(i)}(\omega)}, \omega \epsilon$ $\Omega_{1, j}^{(i)}$,

(c) $f: \Omega_{1, j}^{(i)} \rightarrow \mathbb{R}_{+} \times U, f(\omega):=\left(\zeta_{1, j, \ell}^{(i)}(\omega)\right.$, $\left.p_{1, j}^{(i)}(\omega)\left(\zeta_{1, j, \ell}^{(i)}(\omega)\right)\right), \omega \in \Omega_{1, j}^{(i)}$.

Then there exist functions $\Phi_{j, \ell}: M\left(\mathbb{R}_{+} \times U\right) \rightarrow \mathbb{R}_{+}$ and $\Xi_{j, \ell}: M\left(\mathbb{R}_{+} \times U\right) \rightarrow U$ such that $\Phi_{j, \ell}$ is $\mathscr{M}\left(\mathbb{R}_{+} \times\right.$ $U) / \mathscr{B}\left(\mathbb{R}_{+}\right)$-measurable, $\Xi_{j, \ell}$ is $\mathscr{M}\left(\mathbb{R}_{+} \times U\right) / \mathscr{B}(U)$ measurable, and $\left(\zeta_{1, j, \ell}^{(i)}, p_{1, j}^{(i)}\left(\zeta_{1, j, \ell}^{(i)}\right)\right)=\left(\Phi_{j, \ell}\left(N_{p_{1, j}^{(i)}}\right)\right.$, $\left.\Xi_{j, \ell}\left(N_{p_{1, j}^{(i)}}\right)\right)$ holds on $\Omega_{1, j}^{(i)}$. Since $\mathbb{P}^{(i)}\left(\Omega_{1, j}^{(i)}\right)=1$, we have $\left(\zeta_{1, j, \ell}^{(i)}, p_{1, j}^{(i)}\left(\zeta_{1, j, \ell}^{(i)}\right)\right)=\left(\Phi_{j, \ell}\left(N_{p_{1, j}^{(i)}}\right), \Xi_{j, \ell}\left(N_{p_{1, j}^{(i)}}\right)\right) \mathbb{P}^{(i)}$ almost surely, as desired.

In what follows we provide an alternative argument for verifying that $\zeta_{1, j, \ell}^{(i)}$ is an $\mathscr{M}\left(\mathbb{R}_{+} \times U\right) / \mathscr{B}(\mathbb{R})$ measurable function of $p^{(i)}$ with the advantage that the measurable function in question shows up explicitly. We have $\zeta_{1, j, 1}^{(i)}=\inf \left\{t \in \mathbb{R}_{++}:\left|\Delta y_{i, j}(t)\right|>1 / 2\right\}$, where $y_{i, j}(t):=N^{(i)}\left((0, t] \times U_{1, j}\right)$ and $\Delta y_{i, j}(t):=$ $y_{i, j}(t)-y_{i, j}(t-)=N^{(i)}\left(\{t\} \times U_{1, j}\right)$ for $t \in \mathbb{R}_{++}$. Further, $\zeta_{1, j, \ell+1}^{(i)}=\inf \left\{t \in\left(\zeta_{1, j, \ell}^{(i)}, \infty\right):\left|\Delta y_{i, j}(t)\right|>1 / 2\right\}$ for all $\ell \in \mathbb{N}$. Consider the mappings $\Psi_{3, \ell}: D\left(\mathbb{R}_{+}, \mathbb{R}\right) \rightarrow$ $\mathbb{R}_{+}, \ell \in \mathbb{N}$, defined by $\Psi_{3,1}(y):=\inf \left\{t \in \mathbb{R}_{++}\right.$: $|\Delta y(t)|>1 / 2\}$ and $\Psi_{3, \ell+1}(y):=\inf \left\{t \in\left(\Psi_{3, \ell}(y), \infty\right):\right.$ $|\Delta y(t)|>1 / 2\}, y \in D\left(\mathbb{R}_{+}, \mathbb{R}\right), \ell \in \mathbb{N}$. By Proposition VI.2.7 in Jacod and Shiryaev [17], the mappings $\Psi_{3, \ell}$, $\ell \in \mathbb{N}$, are continuous at each point $y \in D\left(\mathbb{R}_{+}, \mathbb{R}\right)$ such that $|\Delta y(t)| \neq 1 / 2$ for all $t \in \mathbb{R}_{+}$. Moreover, we have $\zeta_{1, j, \ell}^{(i)}=\Psi_{3, \ell}\left(\Psi_{4, j}\left(p^{(i)}\right)\right)$, where the mappings $\Psi_{4, j}: M\left(\mathbb{R}_{+} \times U\right) \rightarrow D\left(\mathbb{R}_{+}, \mathbb{R}\right), j \in \mathbb{N}$, are given by $\Psi_{4, j}(\pi):=\left(\pi\left((0, t] \times U_{1, j}\right)\right)_{t \in \mathbb{R}_{+}}, \pi \in M\left(\mathbb{R}_{+} \times U\right)$. Observe that, for each $\pi \in M\left(\mathbb{R}_{+} \times U\right)$, we have $\left|\Delta \Psi_{4, j}(\pi)(t)\right| \neq 1 / 2$ for all $t \in \mathbb{R}_{+}\left(\right.$since $\left|\Delta \Psi_{4, j}(\pi)(t)\right| \epsilon$ $\mathbb{Z}_{+}$for all $\left.t \in \mathbb{R}_{+}\right)$; hence, it remains to check that the mappings $\Psi_{4, j}, j \in \mathbb{N}$, are $\mathscr{M}\left(\mathbb{R}_{+} \times U\right) / \mathscr{D}\left(\mathbb{R}_{+}, \mathbb{R}\right)$ measurable. This follows from $\left\{\pi \in M\left(\mathbb{R}_{+} \times U\right)\right.$ : $\left.\left(\pi\left((0, t] \times U_{1, j}\right)\right)_{t \in\left\{t_{1}, \ldots, t_{L}\right\}} \in B\right\} \in \mathscr{M}\left(\mathbb{R}_{+} \times U\right)$ for 
all $L \in \mathbb{N}, t_{1}, \ldots, t_{L} \in \mathbb{R}_{+}$and $B \in \mathbb{R}^{L}$, which is a consequence of the definition of $\mathscr{M}\left(\mathbb{R}_{+} \times U\right)$.

(v) Finally, we verify that $I_{4, n, j, \ell}^{(i), a}\left(t_{k}\right)-I_{4, n, j, \ell}^{(i), b}\left(t_{k}\right)$ is a $\mathscr{D}\left(\mathbb{R}_{+}, \mathbb{R}^{d}\right) \otimes \mathscr{M}\left(\mathbb{R}_{+} \times U\right) / \mathscr{B}\left(\mathbb{R}^{d}\right)$-measurable function of $\left(\mathbf{X}^{(i)}, p^{(i)}\right)$. Based on the findings for $I_{1, n}^{(i)}\left(t_{k}\right)$ and $I_{3, n}^{(i)}\left(t_{k}\right)$, it is enough to check that

$$
\begin{aligned}
& \sigma\left(\zeta_{0, j, \ell}^{(i)}, p_{0, j}^{(i)}\left(\zeta_{0, j \ell}^{(i)}\right), \tau_{n}^{(i)}\right) \cap \Omega_{0, j}^{(i)} \\
& \quad \subset \sigma\left(\mathbf{X}^{(i)}, p_{0, j}^{(i)}\right) \cap \Omega_{0, j}^{(i)} \subset \sigma\left(\mathbf{X}^{(i)}, p^{(i)}\right) \cap \Omega_{0, j}^{(i)},
\end{aligned}
$$

where $\zeta_{0, j, \ell}^{(i)}$ and $\Omega_{0, j}^{(i)}$ can be defined similarly as $\zeta_{1, j, \ell}^{(i)}$ and $\Omega_{0, j}^{(i)}$ for all $i \in\{1,2\}$ and $j, \ell \in \mathbb{N}$, respectively (replacing in the definitions $U_{1, j}$ and $p_{1, j}^{(i)}$ by $U_{0, j}$ and $p_{0, j}^{(i)}$, resp.). Note that

$$
\begin{aligned}
& \left\{\omega \in \Omega_{0, j}^{(i)}: \zeta_{0, j, \ell}^{(i)}(\omega) \in(0, t], p_{0, j}^{(i)}(\omega)\left(\zeta_{0, j, \ell}^{(i)}(\omega)\right) \in B,\right. \\
& \left.\tau_{n}^{(i)}(\omega) \in[0, T]\right\} \\
& =\bigcap_{n=1}^{\infty} \bigcup_{k=1}^{n}\left\{\omega \in \Omega_{0, j}^{(i)}:\right. \\
& \quad N_{p_{0, j}^{(i)}(\omega)}\left(\left(0, \frac{(k-1) t}{n}\right] \times U_{0, j}\right) \leqslant \ell-1, \\
& \quad N_{p_{0, j}^{(i)}(\omega)}\left(\left(\frac{(k-1) t}{n}, \frac{k t}{n}\right] \times B\right) \geqslant 1, \\
& \left.N_{p_{0, j}^{(i)}(\omega)}\left(\left(0, \frac{k t}{n}\right] \times U_{0, j}\right) \geqslant \ell\right\} \\
& \bigcap\left\{\omega \in \Omega_{0, j}^{(i)}: \int_{0}^{T} \int_{U_{0}}\left\|f\left(s, \mathbf{X}_{s}^{(i)}(\omega), u\right)\right\|^{2} \mathrm{~d} s m(\mathrm{~d} u) \geq n\right\}
\end{aligned}
$$

for $t \in \mathbb{R}_{++}, T \in \mathbb{R}_{+}, j, \ell \in \mathbb{N}, B \in \mathscr{B}\left(U_{0, j}\right), i \in$ $\{1,2\}$. Similarly, as it was explained in case of $I_{n, 1}^{(i)}(t)$, one can approximate $\int_{0}^{T} \int_{U_{0}}\left\|f\left(s, \mathbf{X}_{s}^{(i)}, u\right)\right\|^{2} \mathrm{~d} s m(\mathrm{~d} u)$ by $\mathscr{D}\left(\mathbb{R}_{+}, \mathbb{R}^{d}\right) / \mathscr{B}\left(\mathbb{R}_{+}\right)$-measurable functions of $\mathbf{X}^{(i)}$, which yields (A.25).

Hence we obtain the statement.

Remark A.3. In case of $f=0$ and $g=0$, the statement of Lemma A.2 basically follows by Exercise (5.16) in Chapter IV in Revuz and Yor [25]; see also Lemma 12.4.5 in von Weizsäcker and Winkler [26].

Next we formulate a corollary of Lemma A.2.

Lemma A.4. Let $\left(\Omega^{(1)}, \mathscr{F}^{(1)},\left(\mathscr{F}_{t}^{(1)}\right)_{t \in \mathbb{R}_{+}}, \mathbb{P}^{(1)}, \mathbf{W}^{(1)}, p^{(1)}, \mathbf{X}^{(1)}\right)$ be a tuple satisfying (D1), (D2), (D3), and (D4)(b)-(d) and let $\left(\Omega^{(2)}, \mathscr{F}^{(2)},\left(\mathscr{F}_{t}^{(2)}\right)_{t \in \mathbb{R}_{+}}, \mathbb{P}^{(2)}, \mathbf{W}^{(2)}, p^{(2)}, \mathbf{X}^{(2)}\right)$ be another tuple satisfying (D1), (D2), and (D3) such that $\left(\mathbf{X}_{t}^{(2)}\right)_{t \in \mathbb{R}_{+}}$is an $\mathbb{R}^{d}$ valued $\left(\mathscr{F}_{t}^{(2)}\right)_{t \in \mathbb{R}_{+}}$-adapted càdlàg process. Suppose that $\left(\mathbf{W}^{(1)}\right.$, $\left.p^{(1)}, \mathbf{X}^{(1)}\right)$ and $\left(\mathbf{W}^{(2)}, p^{(2)}, \mathbf{X}^{(2)}\right)$ have the same distribution on $C\left(\mathbb{R}_{+}, \mathbb{R}^{r}\right) \times M\left(\mathbb{R}_{+} \times U\right) \times D\left(\mathbb{R}_{+}, \mathbb{R}^{d}\right)$. Then (D4)(b)-(d) hold for the tuple $\left(\Omega^{(2)}, \mathscr{F}^{(2)},\left(\mathscr{F}_{t}^{(2)}\right)_{t \in \mathbb{R}_{+}}, \mathbb{P}^{(2)}, \mathbf{W}^{(2)}, p^{(2)}, \mathbf{X}^{(2)}\right)$ as well, and the processes (A.5) and (A.6) have the same distribution on $\left(D\left(\mathbb{R}_{+}, \mathbb{R}^{d}\right)\right)^{5}$.

Proof. First we check that $\mathbb{P}^{(2)}\left(\int_{0}^{t}\left\|b\left(s, \mathbf{X}_{s}^{(2)}\right)\right\| \mathrm{d} s<\infty\right)=1$ for all $t \in \mathbb{R}_{+}$. Since $b$ is $\mathscr{B}\left(\mathbb{R}_{+}\right) \otimes \mathscr{B}\left(\mathbb{R}^{d}\right) \otimes \mathscr{B}(U) / \mathscr{B}\left(\mathbb{R}^{d}\right)$ measurable and $\mathbf{X}^{(1)}$ and $\mathbf{X}^{(2)}$ have the same law, the processes $\left(b\left(s, \mathbf{X}_{s}^{(1)}\right)\right)_{s \in \mathbb{R}_{+}}$and $\left(b\left(s, \mathbf{X}_{s}^{(2)}\right)\right)_{s \in \mathbb{R}_{+}}$have the same law as well. Since the mapping $D\left(\mathbb{R}_{+}, \mathbb{R}^{d}\right) \ni f \mapsto\left(\int_{0}^{t} f(s) \mathrm{d} s\right)_{t \in \mathbb{R}_{+}} \in$ $D\left(\mathbb{R}_{+}, \mathbb{R}^{d}\right)$ is continuous (see, e.g., Ethier and Kurtz [27, Chapter III, Section 11, Exercise 26], or Barczy et al. [28, Proof of Lemma B.3]), and consequently $\mathscr{D}\left(\mathbb{R}_{+}, \mathbb{R}^{d}\right) / \mathscr{D}\left(\mathbb{R}_{+}, \mathbb{R}^{d}\right)$ measurable, the processes $\left(\int_{0}^{t}\left\|b\left(s, \mathbf{X}_{s}^{(1)}\right)\right\| \mathrm{d} s\right)_{t \in \mathbb{R}_{+}}$and $\left(\int_{0}^{t} \| b(s\right.$, $\left.\left.\mathbf{X}_{s}^{(2)}\right) \| \mathrm{d} s\right)_{t \in \mathbb{R}_{+}}$have the same distribution with respect to $\mathbb{P}^{(1)}$ and $\mathbb{P}^{(2)}$, respectively. Since $\mathbb{P}^{(1)}\left(\int_{0}^{t}\left\|b\left(s, \mathbf{X}_{s}^{(1)}\right)\right\| \mathrm{d} s<\infty\right)=1$ for all $t \in \mathbb{R}_{+}$, this yields $\mathbb{P}^{(2)}\left(\int_{0}^{t}\left\|b\left(s, \mathbf{X}_{s}^{(2)}\right)\right\| \mathrm{d} s<\infty\right)=1$ for all $t \in \mathbb{R}_{+}$, as desired.

Similarly, one can check that $\mathbb{P}^{(2)}\left(\int_{0}^{t}\left\|\sigma\left(s, \mathbf{X}_{s}^{(2)}\right)\right\|^{2} \mathrm{~d} s<\right.$ $\infty)=1$ for all $t \in \mathbb{R}_{+}$, and

$$
\mathbb{P}^{(2)}\left(\int_{0}^{t} \int_{U_{0}}\left\|f\left(s, \mathbf{X}_{s}^{(2)}, u\right)\right\|^{2} \mathrm{~d} s m(\mathrm{~d} u)<\infty\right)=1, \quad t \in \mathbb{R}_{+} .
$$

It remains to check that

$$
\mathbb{P}^{(2)}\left(\int_{0}^{t} \int_{U_{1}}\left\|g\left(s, \mathbf{X}_{s-}^{(2)}, u\right)\right\| N^{(2)}(\mathrm{d} s, \mathrm{~d} u)<\infty\right)=1,
$$

for $t \in \mathbb{R}_{+}$, where $N^{(2)}(\mathrm{d} s, \mathrm{~d} u)$ is the counting measure of $p^{(2)}$ on $\mathbb{R}_{++} \times U$. Recall that, in the proof of Lemma A.2, $U_{1, j} \in \mathscr{B}(U), j \in \mathbb{N}$, have been chosen such that they are disjoint, $m\left(U_{1, j}\right)<\infty, j \in \mathbb{N}$, and $U_{1}=\bigcup_{j=1}^{\infty} U_{1, j}$. Further, the set $D\left(p_{1, j}^{(i)}\right)$ is ordered according to magnitude as $0<\zeta_{1, j, 1}^{(i)}<$ $\zeta_{1, j, 2}^{(i)}<\cdots, j \in \mathbb{N}, i \in\{1,2\}$; see (A.9). Then, for each $i \in\{1,2\}$ and $t \in \mathbb{R}_{+}, K_{n}^{(i)}(t) \rightarrow \int_{0}^{t} \int_{U_{1}}\left\|g\left(s, \mathbf{X}_{s^{-}}^{(i)}, u\right)\right\| N^{(i)}(\mathrm{d} s, \mathrm{~d} u)$ as $n \rightarrow \infty \mathbb{P}^{(i)}$-almost surely, where

$$
\begin{aligned}
K_{n}^{(i)}(t) & :=\sum_{j=1}^{n} \int_{0}^{t} \int_{U_{1, j}}\left\|g\left(s, \mathbf{X}_{s-}^{(i)}, u\right)\right\| N^{(i)}(\mathrm{d} s, \mathrm{~d} u) \\
& =\sum_{j=1}^{n} \sum_{s \in(0, t] \cap D\left(p_{1, j}^{(i)}\right)}\left\|g\left(s, \mathbf{X}_{s-}^{(i)}, p_{1, j}^{(i)}(s)\right)\right\|
\end{aligned}
$$

where $p_{1, j}^{(i)}$ denotes the thinning of $p^{(i)}$ onto $U_{1, j}$. Since $\left(p^{(1)}\right.$, $\left.\mathbf{X}^{(1)}\right)$ and $\left(p^{(2)}, \mathbf{X}^{(2)}\right)$ have the same distribution with respect to $\mathbb{P}^{(1)}$ and $\mathbb{P}^{(2)}$, respectively, $K_{n}^{(1)}(t)$ and $K_{n}^{(2)}(t)$ have the same 
distribution with respect to $\mathbb{P}^{(1)}$ and $\mathbb{P}^{(2)}$, respectively, for all $n \in \mathbb{N}$ and $t \in \mathbb{R}_{+}$(which can be checked in the same way as in the proof of Lemma A.2 by replacing $g$ with $\|g\|)$. Consequently, $\int_{0}^{t} \int_{U_{1}}\left\|g\left(s, \mathbf{X}_{s-}^{(1)}, u\right)\right\| N^{(1)}(\mathrm{d} s, \mathrm{~d} u)$ and $\int_{0}^{t} \int_{U_{1}} \| g\left(s, \mathbf{X}_{s-}^{(2)}\right.$, $u) \| N^{(2)}(\mathrm{d} s, \mathrm{~d} u)$ have the same distribution with respect to $\mathbb{P}^{(1)}$ and $\mathbb{P}^{(2)}$, respectively, for all $t \in \mathbb{R}_{+}$. Since

$$
\mathbb{P}^{(1)}\left(\int_{0}^{t} \int_{U_{1}}\left\|g\left(s, \mathbf{X}_{s-}^{(1)}, u\right)\right\| N^{(1)}(\mathrm{d} s, \mathrm{~d} u)<\infty\right)=1,
$$

for $t \in \mathbb{R}_{+}$, we have (A.28). All in all, the tuple $\left(\Omega^{(2)}, \mathscr{F}^{(2)}\right.$, $\left.\left(\mathscr{F}_{t}^{(2)}\right)_{t \in \mathbb{R}_{+}}, \mathbb{P}^{(2)}, \mathbf{W}^{(2)}, p^{(2)}, \mathbf{X}^{(2)}\right)$ satisfies (D4)(b)-(d), and then Lemma A.2 yields that the processes (A.5) and (A.6) have the same distribution on $\left(D\left(\mathbb{R}_{+}, \mathbb{R}^{d}\right)\right)^{5}$. [11].

The next lemma corresponds to Fact B on page 107 in Situ

Lemma A.5. Let us consider the filtered probability space $\left(\Omega, \mathscr{F},\left(\mathscr{F}_{t}\right)_{t \in \mathbb{R}_{+}}, \mathbb{P}_{1,2}\right)$ given in the proof of Theorem 1. The process $\Omega \ni\left(\mathbf{x}, w, \pi, y^{(1)}, y^{(2)}\right) \mapsto w_{t} \in \mathbb{R}^{r}, t \in \mathbb{R}_{+}$, is an $r$-dimensional standard $\left(\mathscr{F}_{t}\right)_{t \in \mathbb{R}_{+}}$-Brownian motion, and the process $\left.\Omega \ni\left(\mathbf{x}, w, \pi, y^{(1)}, y^{(2)}\right) \mapsto N_{p_{\pi}}\right|_{(0, t] \times U} \in M\left(\mathbb{R}_{+} \times U\right)$, $t \in \mathbb{R}_{+}$, is a stationary $\left(\mathscr{F}_{t}\right)_{t \in \mathbb{R}_{+}}$-Poisson point process on $U$ with characteristic measure $m$ under the measure $\mathbb{P}_{1,2}$.

Proof. Using that the $w$-coordinate process is an $r$-dimensional standard $\left(\mathscr{G}_{t}\right)_{t \in \mathbb{R}_{+}}$-Brownian motion under $\mathbb{P}_{1,2}$, for the first statement, it is enough to prove the independence of $w_{t}-w_{s}$ and $\mathscr{F}_{s}$ for every $s, t \in \mathbb{R}_{+}$with $s<t$. For this, it is sufficient to show

$$
\begin{array}{r}
\mathbb{E}_{\mathbb{P}_{1,2}}\left(\mathrm{e}^{\mathrm{i}\left\langle\mathbf{y}, w_{t}-w_{s}\right\rangle} \mathbb{1}_{G}\right)=\mathrm{e}^{-(t-s)\|y\|^{2} / 2} \mathbb{P}_{1,2}(G), \\
\mathbf{y} \in \mathbb{R}^{r}, \quad G \in \mathscr{G}_{s}, \quad 0 \leqslant s<t .
\end{array}
$$

Indeed, if $A \in \widetilde{\mathscr{G}}_{s}$, then there exists some $G \in \mathscr{G}_{s}$ such that $A \Delta G=(A \backslash G) \cup(G \backslash A) \in \mathcal{N}$, and consequently $\mathbb{P}_{1,2}(A \Delta G)=$ 0 . Then,

$$
\begin{aligned}
\mathbb{E}_{\mathbb{P}_{1,2}}\left(\mathrm{e}^{\mathrm{i}\left\langle\mathbf{y}, w_{t}-w_{s}\right\rangle} \mathbb{1}_{A}\right)= & \mathbb{E}_{\mathbb{P}_{1,2}}\left(\mathrm{e}^{\mathrm{i}\left\langle\mathrm{y}, w_{t}-w_{s}\right\rangle} \mathbb{1}_{A \cap G}\right) \\
= & \mathbb{E}_{\mathbb{P}_{1,2}}\left(\mathrm{e}^{\mathrm{i}\left\langle\mathbf{y}, w_{t}-w_{s}\right\rangle} \mathbb{1}_{G}\right) \\
= & \mathrm{e}^{-(t-s)\|y\|^{2} / 2} \mathbb{P}_{1,2}(G) \\
= & \mathrm{e}^{-(t-s)\|y\|^{2} / 2} \mathbb{P}_{1,2}(A), \\
& A \in \widetilde{\mathscr{G}}_{s}, \quad 0 \leqslant s<t .
\end{aligned}
$$

Moreover, if $A \in \mathscr{F}_{s}$, then $A \in \widetilde{\mathscr{G}}_{s+\varepsilon}$ for all $\varepsilon>0$, and hence

$$
\begin{array}{r}
\mathbb{E}_{\mathbb{P}_{1,2}}\left(\mathrm{e}^{\mathrm{i}\left\langle\mathbf{y}, w_{t}-w_{s+\varepsilon}\right\rangle} \mathbb{1}_{A}\right)=\mathrm{e}^{-(t-s-\varepsilon)\|y\|^{2} / 2} \mathbb{P}_{1,2}(A), \\
A \in \mathscr{F}_{s}, \quad 0 \leqslant s<t, \quad \varepsilon>0 .
\end{array}
$$

By dominated convergence theorem, using that $w$ has continuous sample paths $\mathbb{P}_{1,2}$-almost surely, we get

$$
\begin{array}{r}
\mathbb{E}_{\mathbb{P}_{1,2}}\left(\mathrm{e}^{\mathrm{i}\left\langle\mathrm{y}, w_{t}-w_{s}\right\rangle} \mathbb{1}_{A}\right)=\mathrm{e}^{-(t-s)\|y\|^{2} / 2} \mathbb{P}_{1,2}(A), \\
A \in \mathscr{F}_{s}, \quad 0 \leqslant s<t ;
\end{array}
$$

that is,

$$
\mathbb{E}_{\mathbb{P}_{1,2}}\left[\mathrm{e}^{\mathrm{i}\left\langle\mathbf{y}, w_{t}-w_{s}\right\rangle} \mid \mathscr{F}_{s}\right]=\mathrm{e}^{-(t-s)\|y\|^{2} / 2}, \quad 0 \leqslant s<t .
$$

Thus, in the light of Lemma 2.6.13 of Karatzas and Shreve [21], we get the independence of $w_{t}-w_{s}$ and $\mathscr{F}_{s}$ for every $s, t \in \mathbb{R}_{+}$ with $s<t$. obtain

Using that $w_{t}-w_{s}$ is independent of $\mathscr{G}_{s}$ under $\mathbb{P}_{1,2}$, we

$$
\begin{aligned}
\mathbb{E}_{\mathbb{P}_{1,2}}\left[\mathrm{e}^{\mathrm{i}\left\langle\mathbf{y}, w_{t}-w_{s}\right\rangle} \mathbb{1}_{G}\right] & =\mathbb{E}_{\mathbb{P}_{1,2}}\left[\mathbb{E}_{\mathbb{P}_{1,2}}\left[\mathrm{e}^{\mathrm{i}\left\langle\mathbf{y}, w_{t}-w_{s}\right\rangle} \mathbb{1}_{G} \mid \mathscr{G}_{s}\right]\right] \\
& =\mathbb{E}_{\mathbb{P}_{1,2}}\left[\mathbb{1}_{G} \mathbb{E}_{\mathbb{P}_{1,2}}\left[\mathrm{e}^{\mathrm{i}\left\langle\mathbf{y}, w_{t}-w_{s}\right\rangle} \mid \mathscr{G}_{s}\right]\right] \\
& =\mathbb{E}_{\mathbb{P}_{1,2}}\left[\mathbb{1}_{G} \mathbb{E}_{\mathbb{P}_{1,2}}\left[\mathrm{e}^{\mathrm{i}\left\langle\mathbf{y}, w_{t}-w_{s}\right\rangle}\right]\right] \\
& =\mathbb{E}_{\mathbb{P}_{1,2}}\left[\mathbb{1}_{G} \mathrm{e}^{-(t-s)\|y\|^{2} / 2}\right] \\
& =\mathrm{e}^{-(t-s)\|y\|^{2} / 2} \mathbb{P}_{1,2}(G)
\end{aligned}
$$

for all $\mathbf{y} \in \mathbb{R}^{r}$ and $G \in \mathscr{G}_{s}$; hence we conclude (A.31) and then the first statement.

Using that the process $p_{\pi}$ is a stationary $\left(\mathscr{G}_{t}\right)_{t \in \mathbb{R}_{+}}$-Poisson point process on $U$ with characteristic measure $m$, as it was explained in the proof of the first statement, for the second statement, it is enough to show that for every $s, t \in \mathbb{R}_{+}$with $s<t$, every $n \in \mathbb{N}$, every disjoint subsets $B_{1}, \ldots, B_{n} \in \mathscr{B}(U)$ and $\lambda_{1}, \ldots, \lambda_{n} \in \mathbb{R}_{+}$,

$$
\begin{aligned}
& \mathbb{E}_{\mathbb{P}_{1,2}} {\left[\mathrm{e}^{-\sum_{j=1}^{n} \lambda_{j} N_{p_{\pi}}\left((s, t] \times B_{j}\right)} \mathbb{1}_{G}\right] } \\
& \quad=\mathrm{e}^{(t-s) \sum_{j=1}^{n}\left(\mathrm{e}^{-\lambda_{j}}-1\right) m\left(B_{j}\right)} \mathbb{P}_{1,2}(G), \quad G \in \mathscr{G}_{s} .
\end{aligned}
$$

Using that $N_{p_{\pi}}\left((s, t] \times B_{j}\right), j \in\{1, \ldots, n\}$, are independent of each other and from $\mathscr{G}_{s}$ under $\mathbb{P}_{1,2}$, we get

$$
\begin{aligned}
\mathbb{E}_{\mathbb{P}_{1,2}} & {\left[\mathrm{e}^{-\sum_{j=1}^{n} \lambda_{j} N_{p_{\pi}}\left((s, t] \times B_{j}\right)} \mathbb{1}_{G}\right] } \\
= & \mathbb{E}_{\mathbb{P}_{1,2}}\left[\mathbb{E}_{\mathbb{P}_{1,2}}\left[\mathrm{e}^{-\sum_{j=1}^{n} \lambda_{j} N_{p_{\pi}}\left((s, t] \times B_{j}\right)} \mathbb{1}_{G} \mid \mathscr{G}_{s}\right]\right] \\
= & \mathbb{E}_{\mathbb{P}_{1,2}}\left[\mathbb{1}_{G} \mathbb{E}_{\mathbb{P}_{1,2}}\left[\mathrm{e}^{-\sum_{j=1}^{n} \lambda_{j} N_{p_{\pi}}\left((s, t] \times B_{j}\right)} \mid \mathscr{G}_{s}\right]\right] \\
= & \mathbb{E}_{\mathbb{P}_{1,2}}\left[\mathbb{1}_{G} \mathbb{E}_{\mathbb{P}_{1,2}}\left[\mathrm{e}^{-\sum_{j=1}^{n} \lambda_{j} N_{p_{\pi}}\left((s, t] \times B_{j}\right)}\right]\right] \\
= & \mathbb{E}_{\mathbb{P}_{1,2}}\left[\mathbb{1}_{G} \mathrm{e}^{(t-s) \sum_{j=1}^{n}\left(\mathrm{e}^{-\lambda_{j}}-1\right) m\left(B_{j}\right)}\right] \\
= & \mathrm{e}^{(t-s) \sum_{j=1}^{n}\left(\mathrm{e}^{-\lambda_{j}}-1\right) m\left(B_{j}\right)} \mathbb{P}_{1,2}(G)
\end{aligned}
$$

for all $G \in \mathscr{G}_{s}$. The last but one equality above is a consequence that $N_{p_{\pi}}\left((s, t] \times B_{j}\right)$ is a Poisson distributed random variable with parameter $(t-s) m\left(B_{j}\right), j \in\{1, \ldots, n\}$, under $\mathbb{P}_{1,2}$. Hence we conclude the second statement as well. 


\section{Conflict of Interests}

The authors declare that there is no conflict of interests regarding the publication of this paper.

\section{Acknowledgments}

The research of Mátyás Barczy and Gyula Pap was realized in the frames of TÁMOP 4.2.4. A/2-11-1-2012-0001 "National Excellence Program, Elaborating and operating an inland student and researcher personal support system." The project was subsidized by the European Union and cofinanced by the European Social Fund. Zenghu Li has been partially supported by NSFC under Grant no. 11131003 and 973 Program under Grant no. 2011CB808001.

\section{References}

[1] T. Yamada and S. Watanabe, "On the uniqueness of solutions of stochastic differential equations," Journal of Mathematics of Kyoto University, vol. 11, no. 1, pp. 155-167, 1971.

[2] R. M. Dudley, Real Analysis and Probability, The Wadsworth \& Brooks/Cole Mathematics Series, Wadsworth \& Brooks/Cole Advanced Books \& Software, Pacific Grove, Calif, USA, 1989.

[3] H. J. Engelbert, "On the theorem of T. Yamada and S. Watanabe," Stochastics and Stochastics Reports, vol. 36, no. 3-4, pp. 205-216, 1991.

[4] A. S. Cherny, "On strong and weak uniqueness for stochastic differential equations," Theory of Probability and Its Applications, vol. 46, no. 3, pp. 406-419, 2000.

[5] T. G. Kurtz, "Weak and strong solutions of general stochastic models," Electronic Communications in Probability, vol. 19, article 58, 16 pages, 2014.

[6] J. Jacod, "Weak and strong solutions of stochastic differential equations," Stochastics, vol. 3, no. 3, pp. 171-191, 1980.

[7] M. Ondreját, "Uniqueness for stochastic evolution equations in Banach spaces," Dissertationes Mathematicae, vol. 426, 63 pages, 2004.

[8] M. Röckner, B. Schmuland, and X. Zhang, "Yamada-Watanabe theorem for stochastic evolution equations in infinite dimensions," Condensed Matter Physics, vol. 11, no. 2, pp. 247-259, 2008.

[9] S. Tappe, "The Yamada-Watanabe theorem for mild solutions to stochastic partial differential equations," Electronic Communications in Probability, vol. 18, article 24, 13 pages, 2013.

[10] T. G. Kurtz, "The Yamada-Watanabe-Engelbert theorem for general stochastic equations and inequalities," Electronic Journal of Probability, vol. 12, no. 33, pp. 951-965, 2007.

[11] R. Situ, Theory of Stochastic Differential Equations with Jumps and Applications, Mathematical and Analytical Techniques with Applications to Engineering, Springer, New York, NY, USA, 2005.

[12] Z. Li and L. Mytnik, "Strong solutions for stochastic differential equations with jumps," Annales de l'Institut Henri Poincaré, Probabilités et Statistiques, vol. 47, no. 4, pp. 1055-1067, 2011.

[13] D. A. Dawson and Z. Li, "Stochastic equations, flows and measure-valued processes," The Annals of Probability, vol. 40, no. 2, pp. 813-857, 2012.

[14] L. Döring and M. Barczy, "A jump type SDE approach to positive self-similar Markov processes," Electronic Journal in Probability, vol. 17, no. 94, pp. 1-39, 2012.
[15] Z. Li and F. Pu, "Strong solutions of jump-type stochastic equations," Electronic Communications in Probability, vol. 17, article 33, pp. 1-13, 2012.

[16] H. Zhao, "Yamada-Watanabe theorem for stochastic evolution equation driven by Poisson random measure," ISRN Probability and Statistics, vol. 2014, Article ID 982190, 7 pages, 2014.

[17] J. Jacod and A. N. Shiryaev, Limit Theorems for Stochastic Processes, Springer, Berlin, Germany, 2nd edition, 2003.

[18] A. S. Kechris, Classical Descriptive Set Theory, vol. 156 of Graduate Texts in Mathematics, Springer, New York, NY, USA, 1995.

[19] S. I. Resnick, Extreme Values, Regular Variation, and Point Processes, Springer, 2008.

[20] N. Ikeda and S. Watanabe, Stochastic Differential Equations and Diffusion Processes, North-Holland/Kodansha, Amsterdam, The Netherlands, 2nd edition, 1989.

[21] I. Karatzas and S. E. Shreve, Brownian Motion and Stochastic Calculus, Springer, Berlin, Germany, 2nd edition, 1991.

[22] D. L. Cohn, Measure Theory, Birkhäuser, Boston, Mass, USA, 2nd edition, 2013.

[23] D. Applebaum, Lévy Processes and Stochastic Calculus, Cambridge University Press, Cambridge, UK, 2nd edition, 2009.

[24] A. W. van der Vaart, Asymptotic Statistics, Cambridge University Press, 1998.

[25] D. Revuz and M. Yor, Continuous Martingales and Brownian Motion, Springer, Berlin, Germany, 3rd edition, 2001.

[26] H. von Weizsäcker and G. Winkler, Stochastic Integrals, Advanced Lectures in Mathematics, Vieweg, Braunschweig, Germany, 1990.

[27] S. N. Ethier and T. G. Kurtz, Markov Processes, John Wiley \& Sons, New York, NY, USA, 1986.

[28] M. Barczy, M. Ispány, and G. Pap, "Asymptotic behavior of CLS estimators for unstable INAR(2) models," http://arxiv.org/abs/ 1202.1617 . 


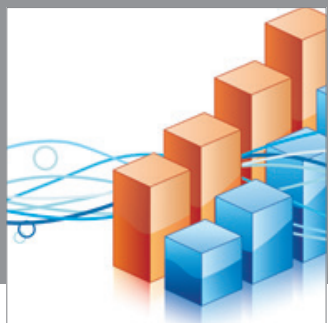

Advances in

Operations Research

mansans

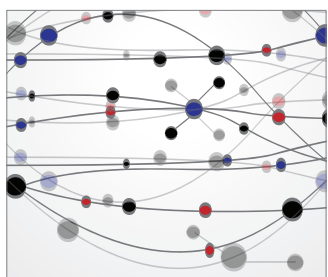

The Scientific World Journal
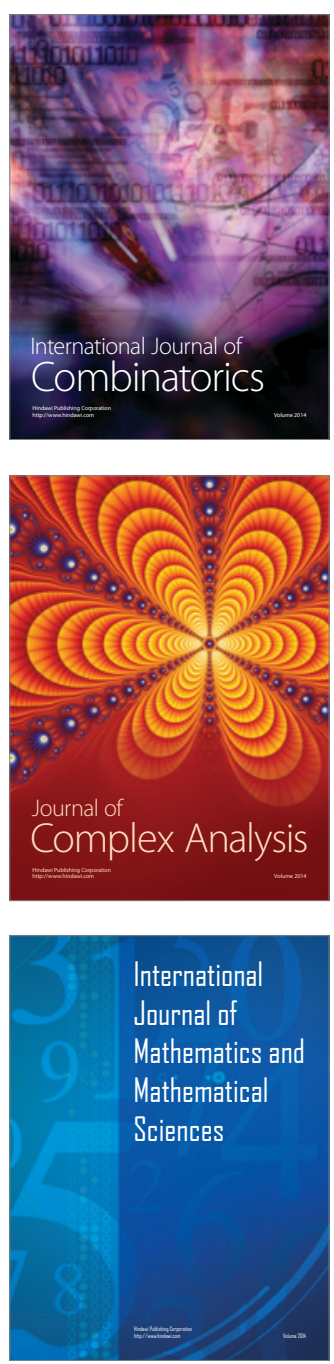
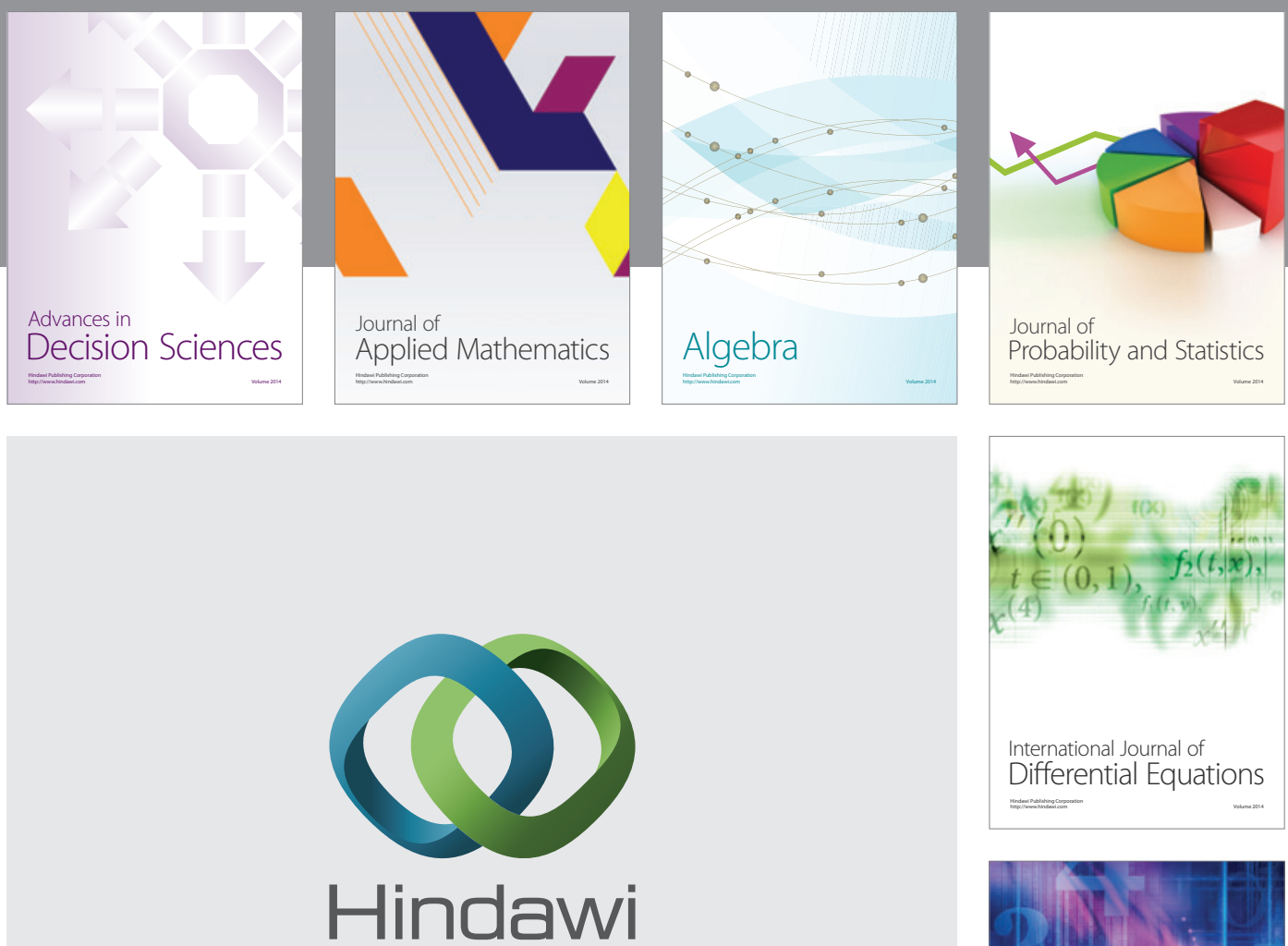

Submit your manuscripts at http://www.hindawi.com
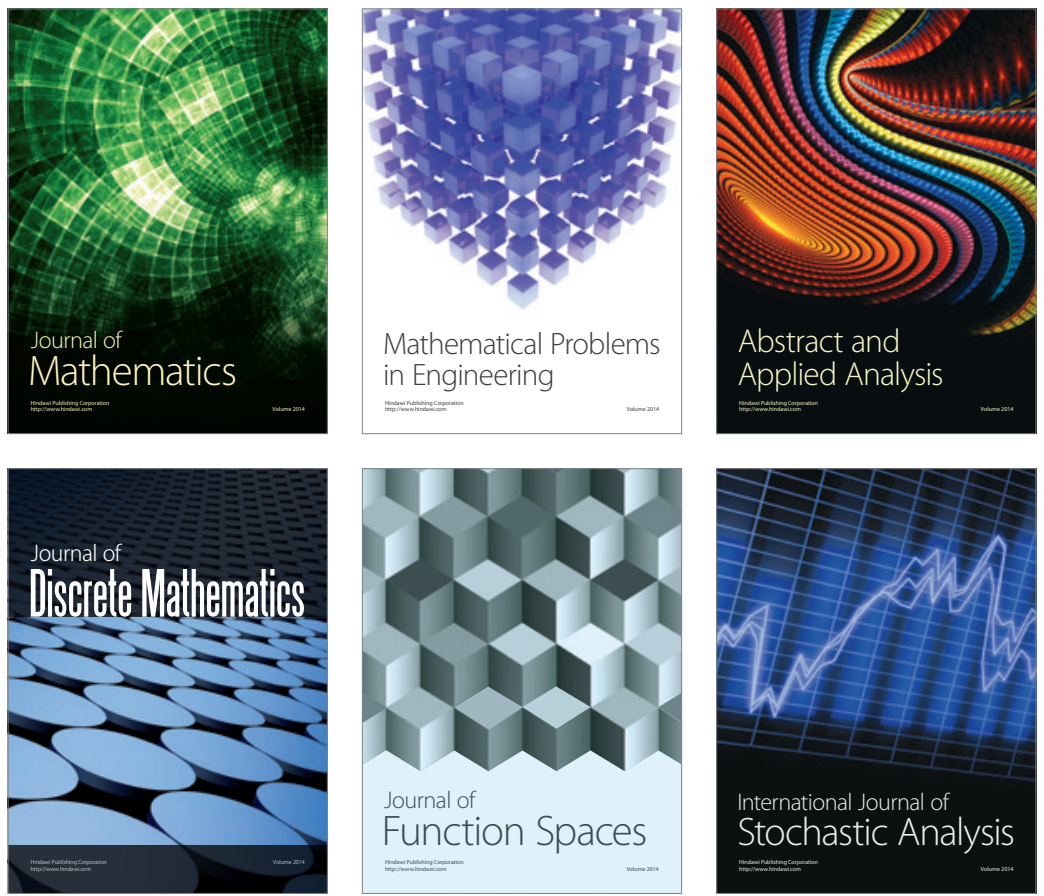

Journal of

Function Spaces

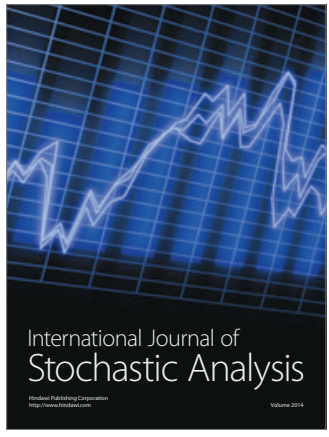

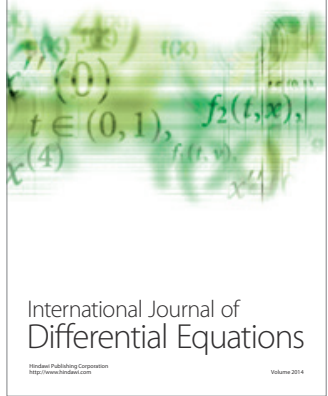
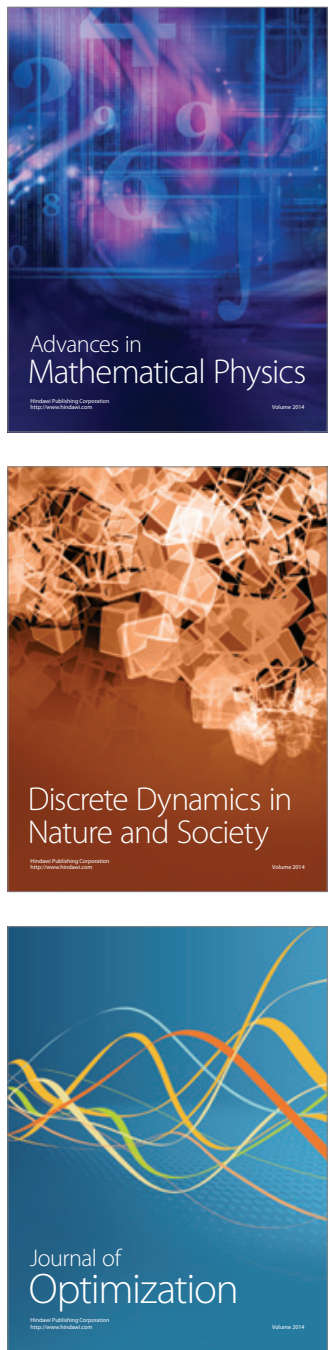